\title{
The dynamical state of the first hydrostatic core candidate Chamaeleon-MMS1 ${ }^{\star}$
}

\author{
A. E. Tsitali ${ }^{1, \star \star}$, A. Belloche ${ }^{1}$, B. Commerçon ${ }^{2}$, and K. M. Menten ${ }^{1}$ \\ ${ }^{1}$ Max-Planck-Institut für Radioastronomie, Auf dem Hügel 69, 53121 Bonn, Germany \\ e-mail: atsitali@mpifr-bonn.mpg.de \\ 2 Laboratoire de radioastronomie, UMR 8112 du CNRS, École Normale Supérieure et Observatoire de Paris, 24 rue Lhomond, \\ 75231 Paris Cedex 5, France
}

Received 31 January 2013 / Accepted 10 June 2013

\begin{abstract}
Context. First hydrostatic cores represent a theoretically predicted intermediate evolutionary link between the prestellar and protostellar phases. Studying the observational characteristics of first core candidates is therefore vital for probing and understanding the earliest phases of star formation.

Aims. We aim to determine the dynamical state of the first hydrostatic core candidate Chamaeleon-MMS1 (Cha-MMS1).

Methods. We observed Cha-MMS1 in various molecular transitions with the APEX and Mopra telescopes. Continuum data retrieved from the Spitzer Heritage Archive were used to estimate the internal luminosity of the source. The molecular emission was modelled with a radiative transfer code to derive constraints on the kinematics of the envelope, which were then compared to the predictions of magneto-hydrodynamic simulations.

Results. We derive an internal luminosity of $0.08 L_{\odot}-0.18 L_{\odot}$ for Cha-MMS1. An average velocity gradient of $3.1 \pm 0.1 \mathrm{~km} \mathrm{~s}^{-1} \mathrm{pc}^{-1}$ over $\sim 0.08 \mathrm{pc}$ is found perpendicular to the filament in which Cha-MMS1 is embedded. The gradient is flatter in the outer parts and, surprisingly, also at the innermost $\sim 2000 \mathrm{AU}$ to $4000 \mathrm{AU}$. The former features are consistent with solid-body rotation beyond $4000 \mathrm{AU}$ and slower, differential rotation beyond $8000 \mathrm{AU}$, but the origin of the flatter gradient in the innermost parts is unclear. The classical infall signature is detected in $\mathrm{HCO}^{+} 3-2$ and CS $2-1$. The radiative transfer modelling indicates a uniform infall velocity in the outer parts of the envelope. In the inner parts (at most $9000 \mathrm{AU}$ ), an infall velocity field scaling with $r^{-0.5}$ is consistent with the data, but the shape of the profile is less well constrained and the velocity could also decrease toward the centre. The infall velocities are subsonic to transonic, $0.1 \mathrm{~km} \mathrm{~s}^{-1}-0.2 \mathrm{~km} \mathrm{~s}^{-1}$ at $r \geq 3300 \mathrm{AU}$, and subsonic to supersonic, $0.04 \mathrm{~km} \mathrm{~s}^{-1}-0.6 \mathrm{~km} \mathrm{~s}^{-1}$ at $r \leq 3300$ AU. Both the internal luminosity of Cha-MMS1 and the infall velocity field in its envelope are consistent with predictions of MHD simulations for the first core phase. There is no evidence of any fast, large-scale outflow stemming from Cha-MMS1, but excess emission from the high-density tracers CS 5-4, CO 6-5, and CO 7-6 suggests the presence of higher velocity material at the inner core.

Conclusions. Its internal luminosity excludes Cha-MMS1 being a prestellar core. The kinematical properties of its envelope are consistent with Cha-MMS1 being a first hydrostatic core candidate or a very young Class 0 protostar.
\end{abstract}

Key words. stars: protostars - stars: formation - stars: low-mass - stars: kinematics and dynamics - ISM: kinematics and dynamics

\section{Introduction}

Many advances have recently been made in the field of early low-mass star formation, spanning from the prestellar phase to the formation and evolution of Young Stellar Objects (YSOs), e.g., with the Spitzer c2d Legacy Project ("From Molecular Cores to Planet-Forming Disks", Evans et al. 2003) and the Herschel Gould Belt Survey (André et al. 2010). In particular, Herschel (Pilbratt et al. 2010) has provided valuable insight into the early star formation processes. Most dense starless cores in molecular clouds appear to be located along a complex network of long, thin filaments, suggesting that filament formation precedes the core formation process (Arzoumanian et al. 2011; Hill et al. 2011; André et al. 2010; Men'shchikov et al. 2010; Molinari et al. 2010). The study of these early stages is necessary

^ Tables 6-9, 11, Figs. 2, 3, 12, 13, 15, 16, 18, 19, and Appendices are available in electronic form at http://www . aanda.org

$\star \star$ Member of the International Max Planck Research School (IMPRS) for Astronomy and Astrophysics at the Universities of Bonn and Cologne. in order to address open questions such as the origin of the stellar initial mass function (IMF), its relationship with the prestellar phase and the core mass function (CMF; e.g., André et al. 2009), and the initial conditions needed for star formation to occur. Recent results from the Herschel survey confirm the resemblance of the prestellar CMF to the stellar IMF in the Aquila and Polaris clouds (Könyves et al. 2010; André et al. 2010). Such a resemblance has already been seen in various molecular clouds with ground-based single-dish telescopes, such as in the Ophiuchus molecular cloud (Motte et al. 1998) or in the Pipe nebula (Rathborne et al. 2009).

It has recently become common to split the population of the starless cores in molecular clouds into two categories, the gravitationally bound and unbound cores. Prestellar cores represent the subset of starless cores that are self-gravitating and will thus very likely form stars (e.g., di Francesco et al. 2007; André et al. 2009), while the gravitationally unbound starless cores may be transient objects ("failed" cores) or objects on the verge of becoming prestellar (e.g. Belloche et al. 2011b). The gravitational collapse of a prestellar core leads to the formation of a stellar 
embryo, the protostar. This marks the beginning of the Class 0 phase, during which the central object accretes mass from its protostellar envelope (André et al. 2000). Theoretically, the early work of Larson (1969) already showed that the formation of the central protostar must be preceded by the formation of a larger, less dense, first hydrostatic core (FHSC). The FHSC thus represents an intermediate evolutionary stage between the prestellar and protostellar phases. The detection of FHSCs is observationally very challenging because of their very short expected lifetime.

\subsection{FHSC: a theoretical background}

The formation of the first hydrostatic core emerged from theory for the first time with Larson (1969), but only a handful of objects have recently been observed and suggested as likely candidates. Larson (1969) describes the process of forming a protostar from a parent molecular core using a spherical collapse model that ignores magnetic fields and rotation. The initial phase is characterised by an isothermal contraction of the molecular core. When the central density exceeds $10^{-13} \mathrm{~g} \mathrm{~cm}^{-3}$ the radiative cooling ceases to be efficient and an opaque, adiabatic core forms at the centre. The rise in temperature results in an increase in the thermal pressure, and finally, when the pressure balances the gravitational force the collapse ceases and the first hydrostatic core is formed. The initial central temperature of the FHSC is estimated to be around $170 \mathrm{~K}$ with an initial central density of $2 \times 10^{-10} \mathrm{~g} \mathrm{~cm}^{-3}$. The so-called second, more compact (protostellar) core is formed after the dissociation of $\mathrm{H}_{2}$ and subsequent collapse, when the central temperature reaches $2 \times 10^{4} \mathrm{~K}$ and the density reaches $2 \times 10^{-2} \mathrm{~g} \mathrm{~cm}^{-3}$ (Larson 1969).

Various theoretical studies predict observational characteristics of the first core phase. Internal luminosities of up to $\sim 0.1 L_{\odot}$ (Masunaga et al. 1998; Saigo \& Tomisaka 2011) or $\sim 0.25 L_{\odot}$ (Commerçon et al. 2012) have been predicted. First cores are characterised by radii and masses in the ranges of $\sim 5 \mathrm{AU}-10 \mathrm{AU}$ and $0.05 M_{\odot}-0.1 M_{\odot}$, respectively (Masunaga et al. 1998; Saigo et al. 2008). Their lifetimes range from a few $100 \mathrm{yr}$ to a few $1000 \mathrm{yr}$, increasing with the rate of rotation. Commerçon et al. (2012) derive lifetimes ranging from $\sim 1000 \mathrm{yr}$ to $>4000 \mathrm{yr}$ for rotating, magnetised $1 M_{\odot}$ cores with 3D radiation-MHD simulations. The FHSC lifetime is shorter for higher levels of magnetisation owing to the stronger magnetic braking that increases the mass accretion rate. These short lifetimes imply that first cores are rare and thus difficult to observe, although we note that Tomida et al. (2010) predict much longer lifetimes $\left(>10^{4} \mathrm{yr}\right)$ for first cores formed in very low-mass cloud cores $\left(0.1 M_{\odot}\right)$.

Outflows at the first core phase are thought to be a significant observational signature characterising this evolutionary stage. Machida et al. (2008) used 3D resistive MHD simulations to study the driving mechanisms of outflows in the star formation process. Their predictions distinguish between an extended, slow molecular outflow driven by the first core and a highly collimated, fast jet later driven by the protostellar core that exhibit typical velocities of $\sim 3 \mathrm{~km} \mathrm{~s}^{-1}$ and $30 \mathrm{~km} \mathrm{~s}^{-1}$, respectively. The outflow driven by the first core is predicted to be extremely compact, spanning 200 AU-800 AU in extent just before the start of the second collapse (Commerçon et al. 2012, 2010; Machida et al. 2008). The first core outflows are thought to result from the twisting of the magnetic field lines due to the rotation of the collapsing core, whose amplified toroidal component leads to the subsequent transfer of angular momentum to the gas outside of the core (Tomisaka \& Tomida 2011).

\subsection{Cha-MMS1 and its evolutionary stage}

Chamaeleon-MMS1 (hereafter Cha-MMS1 for short) is a dense core embedded in a filament $(\sim 0.5 \mathrm{pc}$ in length) within the Chamaeleon I molecular cloud (Belloche et al. 2011a) at a distance of 150 pc (Whittet et al. 1997; Knude \& Høg 1998). Several previous studies of Cha-MMS1 suggest that it is an object at a very early evolutionary stage.

Reipurth et al. (1996) discovered Cha-MMS1 in dust continuum emission at $1.3 \mathrm{~mm}$. They suggested that Cha-MMS1 is the driving source of the nearby HH 49/50 objects and identified it as a Class 0 protostar based on that association. During the Class 0 protostellar phase, the central object is deeply embedded within its collapsing envelope, which comprises more than half of the system's mass (André et al. 1993). Lehtinen et al. (2001) confirms this classification based on a tentative far-infrared detection, but Lehtinen et al. (2003) argues that Cha-MMS1 possibly represents an evolutionary stage earlier than Class 0 based on its lack of thermal free-free emission at $\mathrm{cm}$ wavelengths. Cha-MMS1 is embedded in a gravitationallybound $\mathrm{C}^{18} \mathrm{O}$ core (Haikala et al. 2005). High deuterium fractionations of $\mathrm{HCO}^{+}$and $\mathrm{N}_{2} \mathrm{H}^{+}$were derived, consistent with Cha-MMS1 being an evolved prestellar core or a young protostellar envelope (Belloche et al. 2006).

A faint Spitzer $24 \mu \mathrm{m}$ and $70 \mu \mathrm{m}$ detection indicates the presence of a central object in Cha-MMS1, either an FHSC or a protostar (Belloche et al. 2006). Belloche et al. (2011a) derived a very low internal luminosity of $\sim 0.015 L_{\odot}$ for this object based on the correlation between the $70 \mu \mathrm{m}$ flux density and internal luminosity established by Dunham et al. (2008) for protostellar objects. As most Class 0 protostars feature an outflow (André et al. 2000), a search for a large-scale outflow driven by Cha-MMS1 was performed in CO 3-2 with APEX but none was found (Belloche et al. 2006). The non-detection of an outflow around Cha-MMS1 on scales of $\sim 10^{4}$ AU suggests that the central object may be less evolved than a Class 0 object. It could possess an outflow that is too compact to have been detected with the resolution of the previous studies, which would agree with the predictions of the FHSC observational signatures. Furthermore, Cha-MMS1, the Class 0 protostar IRAM 04191+1522 (hereafter, IRAM 04191) and the very low luminosity object (VeLLO) L1521F (Crapsi et al. 2004; Bourke et al. 2006) are located at approximately the same distance, and a direct comparison of their Spitzer fluxes supports the idea that Cha-MMS1 is less evolved (Belloche et al. 2006). Bearing all this in mind, Cha-MMS1 could be at the stage of the FHSC between the prestellar and Class 0 phases. However, its classification as such is very difficult to observationally confirm until a compact (200 AU-800 AU; Commerçon et al. 2010, 2012), slow outflow with velocities in the range of $2 \mathrm{~km} \mathrm{~s}^{-1}-4 \mathrm{~km} \mathrm{~s}^{-1}$ (Tomisaka 2002; Machida et al. 2008; Commerçon et al. 2010; Hennebelle \& Fromang 2008) is detected. We therefore merely consider it as an FHSC candidate.

\subsection{FHSC candidates}

The detection of seven candidate first cores has been claimed so far: Cha-MMS1 (Belloche et al. 2006, 2011a), L1448-IRS2E (Chen et al. 2010), Per-Bolo 58 (Enoch et al. 2010; Dunham et al. 2011), L1451-mm (Pineda et al. 2011), CB17-MMS (Chen et al. 2012), B1-bS, and B1-bN (Pezzuto et al. 2012). All but one (B1-bS) are VeLLOs (internal luminosity $L_{\text {int }}<0.1 L_{\odot}$ ), in agreement with the range of luminosities predicted for FHSCs. With $L_{\text {bol }} \sim 0.49 L_{\odot}$, B1-bS might be too luminous for an FHSC. 
L1448-IRS2E, Per-Bolo 58, and L1451-mm drive outflows that have been interferometrically detected. L1448-IRS2E drives an outflow with velocities of $\sim 25 \mathrm{~km} \mathrm{~s}^{-1}$, one order of magnitude higher than predicted for a first core by MHD simulations (Machida et al. 2008). This suggests that it is at the more evolved second-core stage so is likely ruled out as a first core candidate. Per-Bolo 58, L1451-mm, and CB17-MMS all have outflow velocities in agreement with theoretical predictions (see Sect. 1.1). However, the outflows of Per-Bolo 58 and CB17-MMS extend over $6000 \mathrm{AU}-8000 \mathrm{AU}$, with dynamical times $\sim 10^{4} \mathrm{yr}$, about one order of magnitude longer than the expected first core lifetime in a magnetised collapsing dense core. Lifetimes in the range $\sim 4000 \mathrm{yr}-10000 \mathrm{yr}$ are produced by non- or very-weakly magnetised simulations, but no outflow is produced at the FHSC stage in these cases (e.g, Commerçon et al. 2012). L1451-mm is therefore the only candidate driving an outflow with properties (maximum velocity $2.3 \mathrm{~km} \mathrm{~s}^{-1}$, dynamical time $1.6 \times 10^{3} \mathrm{yr}$ ) consistent with current theoretical predictions at the first-core stage. There has been no outflow detection for the B1-bS and B1-bN condensations in Perseus, but the SED fitting of their Herschel and Spitzer (where applicable) fluxes seems to be consistent with the presence of a central object surrounded by a dusty envelope that is younger than the Class 0 phase (Pezzuto et al. 2012).

The goal of this study is to set constraints on the kinematics of the envelope of Cha-MMS1 to test that it is consistent with Cha-MMS1 being in the FHSC stage. The structure of this paper is as follows. In Sect. 2 we summarise the observational details, we then present our results in Sect. 3 and in Sect. 4 we perform radiative transfer modelling of the spectra towards Cha-MMS1. The discussion and conclusions follow in Sects. 5 and 6 , respectively.

\section{Observations}

We performed observations towards the dense core Cha-MMS1 at $\alpha_{2000}=11^{\mathrm{h}} 06^{\mathrm{m}} 33^{\mathrm{s}} .13, \delta_{2000}=-77^{\circ} 23^{\prime} 35.1^{\prime \prime 1}$ with the APEX and Mopra telescopes on the central core position as well as on offset positions close to the core along directions parallel and perpendicular to the filament in which it is embedded (see Fig. 1). The data were reduced with the CLASS software ${ }^{2}$.

\subsection{APEX observations}

Observations with $\mathrm{APEX}^{3}$ using the $\mathrm{CHAMP}^{+4}$ heterodyne SSB receiver were carried out in 2010 July, in the following molecular transitions: ${ }^{13} \mathrm{CO} 6-5, \mathrm{CO} 6-5$, and $\mathrm{CO} 7-6$. $\mathrm{CHAMP}^{+}$is a $2 \times 7$ pixel array receiver connected to a fastfourier-transform spectrometer backend array (FFTS). It operates in two frequency bands simultaneously, around $690 \mathrm{GHz}$ and $810 \mathrm{GHz}$. The channel spacing is $183 \mathrm{kHz}$. The corresponding velocity resolution for each transition is given in Table 1. All three transitions were observed with the central $\mathrm{CHAMP}^{+}$pixel pointed on the central core position at $\alpha_{J 2000}=$ $11^{\mathrm{h}} 06^{\mathrm{m}} 33^{\mathrm{s}} .13, \delta_{J 2000}=-77^{\circ} 23^{\prime} 35.1^{\prime \prime}$. In addition to the central position, $\mathrm{CO}$ 6-5 and $\mathrm{CO} 7-6$ were also observed with the central $\mathrm{CHAMP}^{+}$pixel being centred on an offset position

\footnotetext{
1 Position from Spitzer observations (Belloche et al. 2011a).

2 See http://www.iram. fr/IRAMFR/GILDAS

3 The Atacama Pathfinder Experiment telescope (APEX) is a collaboration between the Max-Planck Institut für Radioastronomie, the European Southern Observatory, and the Onsala Space Observatory.

4 See http://www3.mpifr-bonn.mpg.de/div/submmtech/ heterodyne/champplus/champmain.html
}

$(\Delta \alpha, \Delta \delta)=\left(5.3^{\prime \prime}, 8.3^{\prime \prime}\right)$ relative to the centre of Cha-MMS1 (see Fig. 1b). The observations were done in position-switching mode with the reference position at $(\Delta \alpha, \Delta \delta)=\left(-600^{\prime \prime}, 4^{\prime \prime}\right)$. The reference position was checked to be free of emission with an rms sensitivity of $0.06 \mathrm{~K}$ and $0.24 \mathrm{~K}$ for the central pixel in $\mathrm{CO}$ 6-5 and CO 7-6, respectively, and for the spectral resolution given in Table 1.

The observations were carried out on four different days, in the last two of which ${ }^{13} \mathrm{CO} 6-5$ was observed in parallel to CO 7-6. A comparison to the CO 7-6 spectra of the first two days of observation suggests that there is a pointing offset in the south-west direction parallel to the filament of up to $\sim 5^{\prime \prime}$ between the $\mathrm{CO} 6-5 / \mathrm{CO} 7-6$ and ${ }^{13} \mathrm{CO} 6-5 / \mathrm{CO} 7-6$ datasets. The forward and beam efficiencies used to convert antenna temperatures $T_{\mathrm{a}}{ }^{\star}$ into main-beam temperatures can be found in Table 1 along with further information on line frequencies, system temperatures, and noise levels. The focus was optimised on Saturn and Mars and the pointing in CO 6-5 emission on the star IRAS 07454-7112.

\subsection{APEX observations}

We carried out observations with the APEX telescope in 2011 April, June, and December in the following molecular transitions: CS 5-4, $\mathrm{H}^{13} \mathrm{CO}^{+} 3-2, \mathrm{HCO}^{+} 3-2, \mathrm{H}^{13} \mathrm{CO}^{+} 4-3, \mathrm{CO} 4-3$, $\mathrm{C}^{18} \mathrm{O} 2-1$, and $\mathrm{C}^{17} \mathrm{O} 2-1$. The observations were done in position-switching mode with the reference position at $\alpha_{J 2000}=$ $11^{\mathrm{h}} 05^{\mathrm{m}} 23.7, \delta_{J 2000}=-77^{\circ} 11^{\prime} 02.2^{\prime \prime}$. The reference position is free of emission with an rms of $0.12 \mathrm{~K}^{\text {in } \mathrm{HCO}^{+}} 3-2$ and $0.33 \mathrm{~K}$ and $\mathrm{CO} 4-3$ for the spectral resolution given in Table 2. The corresponding beamwidth, forward, and main beam efficiencies are listed in Table 2. The positions observed for each transition are shown in Figs. 1c to e, overlaid on the $870 \mu \mathrm{m}$ map of the filament seen in Fig. 1a. All positions lie either perpendicular (position angle $-35^{\circ}$ east from north) or parallel to the filament. The transitions CS 5-4, $\mathrm{H}^{13} \mathrm{CO}^{+} 4-3, \mathrm{CO} 4-3, \mathrm{C}^{18} \mathrm{O} 2-1$, and $\mathrm{C}^{17} \mathrm{O} 2-1$ were only observed perpendicular to the filament, while $\mathrm{H}^{13} \mathrm{CO}^{+} 3-2$ and $\mathrm{HCO}^{+} 3-2$ were also observed parallel to it. Table 2 gives information about the frontend and backend used for each transition, their respective spectral resolutions, the resulting system temperatures, and rms noise levels. The telescope pointing was checked every $1 \mathrm{~h}$ to $1.5 \mathrm{~h}$ and was performed on IRAS 07454-7112. The pointing accuracy is $\sim 2^{\prime \prime}$ (rms). The focus was optimised on either Saturn, Jupiter, or Mars, and repeated approximately every $3 \mathrm{~h}$.

\subsection{Mopra observations}

We observed with the Mopra telescope towards the central position of Cha-MMS1 along with eight other offset positions perpendicular and parallel to the filament (see Fig. 1f) in 2011 May in several molecular transitions using the zoom mode of the high-resolution spectrometer MOPS. The receiver was tuned at two different frequencies, $94554 \mathrm{MHz}$ and $87190 \mathrm{MHz}$. Only the central position was observed for the latter. Positionswitching observations were done with the reference position at $\alpha_{J 2000}=11^{\mathrm{h}} 05^{\mathrm{m}} 23.7, \delta_{J 2000}=-77^{\circ} 11^{\prime} 02.2^{\prime \prime}$. It was checked that it was free of emission with an rms sensitivity ranging from $46 \mathrm{mK}-52 \mathrm{mK}$ in all transitions for the spectral resolution given in Table 3. The transitions that are used for the analysis in this paper are listed here: CS $2-1, \mathrm{C}^{34} \mathrm{~S} 2-1,{ }^{13} \mathrm{CS} 2-1$, $\mathrm{C}^{33} \mathrm{~S} 2-1, \mathrm{HC}_{3} \mathrm{~N} 10-9, \mathrm{HN}^{13} \mathrm{C} 1-0, \mathrm{~N}_{2} \mathrm{H}^{+} 1-0, \mathrm{HCO}^{+} 1-0$, $\mathrm{H}^{13} \mathrm{CO}^{+} 1-0, \mathrm{HC}^{18} \mathrm{O}^{+} 1-0, \mathrm{HNCO} 4-3, \mathrm{c}-\mathrm{C}_{3} \mathrm{H}_{2} 3_{2,2}-3_{1,3}$, 

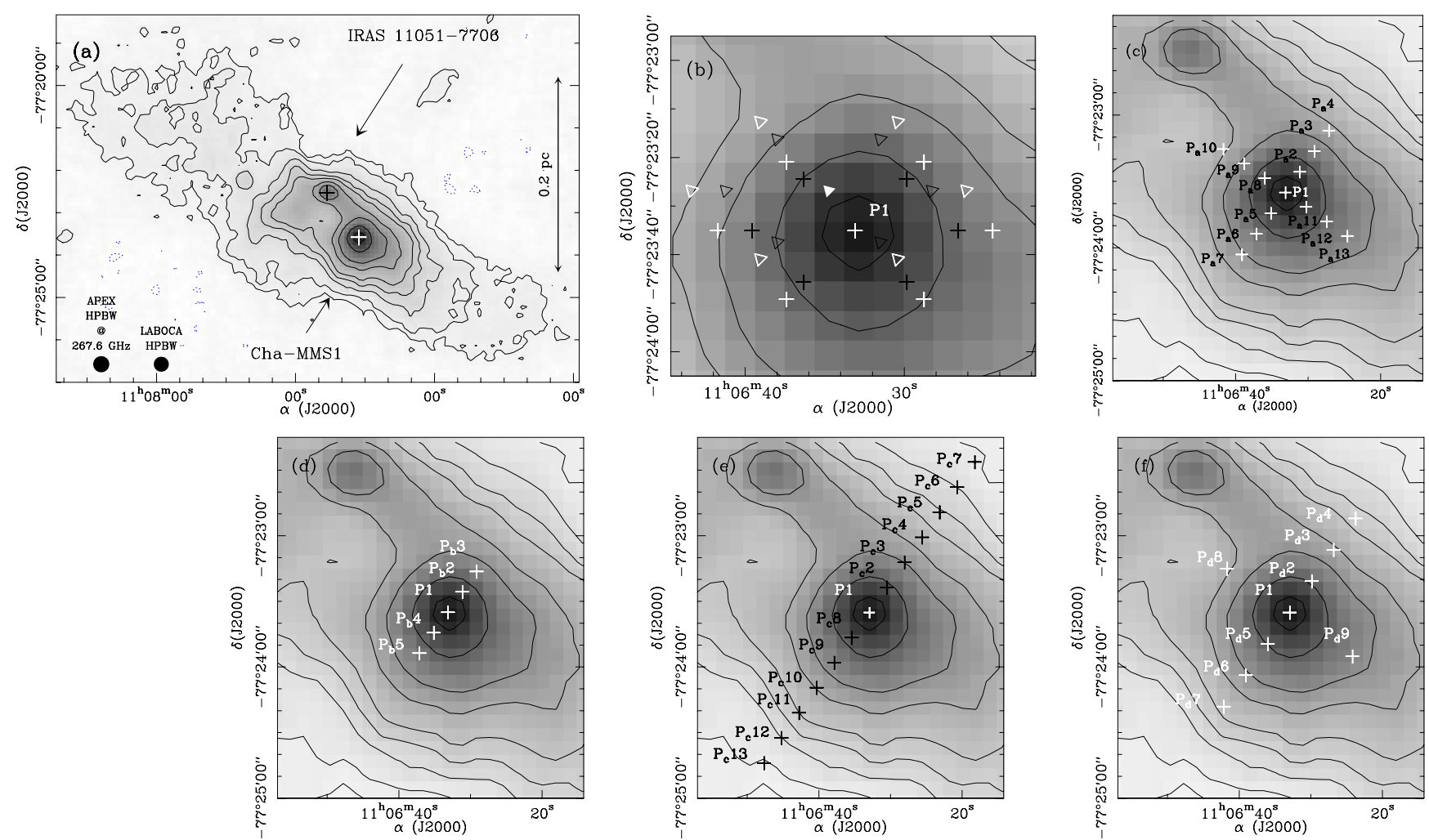

Fig. 1. a) $870 \mu \mathrm{m}$ map of the filament in which Cha-MMS1 is embedded, obtained with LABOCA as part of an unbiased survey of Chamaeleon I

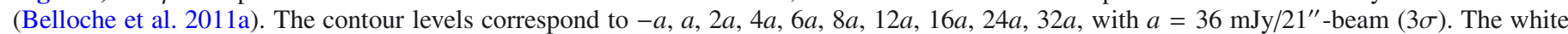
cross at $\alpha_{J 2000}=11^{\mathrm{h}} 06^{\mathrm{m}} 33^{\mathrm{s}} 13, \delta_{J 2000}=-77^{\circ} 23^{\prime} 35.1^{\prime \prime}$ is the Spitzer position of Cha-MMS1. The position of the nearby Class I object IRAS $11051-$ 7706 is also shown with a black cross. b)-f) Zoom-in of the positions (P, white/black crosses/triangles) observed with APEX and Mopra. $\mathrm{P}_{1}$ is the central position. Panel b) refers to $\mathrm{CO} 6-5$ (white crosses), ${ }^{13} \mathrm{CO} 6-5$ (white crosses), and $\mathrm{CO} 7-6$ (black crosses); $\mathbf{c}$ ) to $\mathrm{HCO}^{+} 3-2$ and $\mathrm{H}^{13} \mathrm{CO}^{+} 3-2$; d) to $\mathrm{CS} 5-4, \mathrm{H}^{13} \mathrm{CO}^{+} 4-3$, and $\mathrm{CO} 4-3$; e) to $\mathrm{C}^{17} \mathrm{O} 2-1$ and $\mathrm{C}^{18} \mathrm{O} 2-1$ (all with APEX); and f) to the Mopra observations (see Table 3). The spacing between adjacent positions is $11.4^{\prime \prime}$ in panels c) and $\mathbf{d}$ ), and $14^{\prime \prime}$ in panel e). In panel $\mathbf{f}$ ), it is $17.5^{\prime \prime}$ for the positions perpendicular to the filament and $35^{\prime \prime}$ for the positions along the filament. For panel b) the pixel spacing of the $\mathrm{CHAMP}^{+}$array is $20^{\prime \prime}$ for the white and $15^{\prime \prime}$ for the black crosses relative to P1. The black (CO 7-6) and white (CO 6-5) open triangles mark the positions observed with $\mathrm{CHAMP}^{+}$centred on the offset position at $\left(5.3^{\prime \prime}, 8.3^{\prime \prime}\right)$ relative to Cha-MMS1 (filled triangle).

Table 1. Parameters of 2010 APEX CHAMP ${ }^{+}$observations.

\begin{tabular}{lcccccccccc}
\hline \hline Transition & $\begin{array}{c}f^{a} \\
(\mathrm{MHz})\end{array}$ & $\begin{array}{c}\sigma_{v}{ }^{b} \\
(\mathrm{kHz})\end{array}$ & $\begin{array}{c}H P B W^{c} \\
\left({ }^{\prime \prime}\right)\end{array}$ & $\begin{array}{c}\delta f^{d} \\
(\mathrm{kHz})\end{array}$ & $\begin{array}{c}\delta V^{e} \\
\left(\mathrm{~km} \mathrm{~s}^{-1}\right)\end{array}$ & $N_{\mathrm{pos}^{f}}$ & $\begin{array}{c}F_{\mathrm{eff}^{g}} \\
(\%)\end{array}$ & $\begin{array}{c}B_{\mathrm{eff}^{g}} \\
(\%)\end{array}$ & $\begin{array}{c}T_{\text {sys }^{h}} \\
(\mathrm{~K})\end{array}$ & $\begin{array}{c}\mathrm{rms}^{i} \\
(\mathrm{mK})\end{array}$ \\
\hline${ }^{13}$ CO 6-5 & 661067.2766 & 0.5 & 9.2 & 183 & 0.083 & 7 & 95 & 36 & $1000-1200$ & $76-85$ \\
CO 6-5 & 691473.0763 & 0.5 & 8.8 & 183 & 0.079 & 14 & 95 & 36 & $900-1900$ & $108-185$ \\
CO 7-6 & 806651.8060 & 5.0 & 7.5 & 183 & 0.068 & 14 & 95 & 36 & $2200-6900$ & $217-608$ \\
\hline
\end{tabular}

Notes. ${ }^{(a)}$ Rest frequency taken from the Cologne Database for Molecular Spectroscopy (CDMS, http://www.astro. uni-koeln.de/cdms). ${ }^{(b)}$ Frequency uncertainty taken from the CDMS catalogue. ${ }^{(c)}$ Angular resolution. ${ }^{(d)}$ Channel spacing in frequency. ${ }^{(e)}$ Channel spacing in velocity. ${ }^{(f)}$ Number of observed positions. ${ }^{(g)}$ Forward and main-beam efficiencies from CHAMP ${ }^{+}$webpage. ${ }^{(h)}$ System temperature. ${ }^{(i)}$ rms sensitivity in $T_{\mathrm{a}}^{\star}$ scale.

$\mathrm{CH}_{3} \mathrm{OH}-A 2_{0,2}-1_{0,1}$, and $\mathrm{CH}_{3} \mathrm{OH}-E 2_{1,2}-1_{1,1}$. The final reduced dataset was obtained after averaging both polarisations. We did, however, notice some differences in integrated intensity for the two polarisations of up to $\sim 10 \%$ (see Appendix A for more details about this discrepancy). The channel spacing was $34 \mathrm{kHz}$. The range of system temperatures $T_{\text {sys }}$ for each transition is given in Table 3 . The beam efficiency used to convert antenna temperatures $T_{\mathrm{a}}{ }^{\star}$ into main-beam temperatures is 0.34 . This value was derived from a detailed calibration analysis (see Appendix A). The telescope pointing was checked approximately every hour on U Men for Cha-MMS1, and AH Sc and IK Tau for the calibration sources Oph A SM1N and IRAM 04191, respectively. The transitions, their rest frequencies, and the number of observed positions are listed in Table 3 .

\subsection{Spitzer archive data}

We used MIPS1 $24 \mu \mathrm{m}$ and MIPS2 $70 \mu \mathrm{m}$ continuum data taken from the Spitzer Heritage Archive ${ }^{5}$ (AORkeys: 19978496, 3962112, 19979264).

\footnotetext{
5 See http://irsa.ipac.caltech.edu/data/SPITZER/ docsspitzerdataarchives/
} 
Table 2. Parameters of 2011 APEX observations.

\begin{tabular}{|c|c|c|c|c|c|c|c|c|c|c|c|c|}
\hline Transition & $\underset{(\mathrm{MHz})}{f^{a}}$ & $\begin{array}{l}\sigma_{v}^{b} \\
(\mathrm{kHz})\end{array}$ & $\begin{array}{c}H P B W^{c} \\
(")\end{array}$ & Receiver & Backend & $\begin{array}{l}\delta f^{d} \\
(\mathrm{kHz})\end{array}$ & $\begin{array}{c}\delta V^{e} \\
\left(\mathrm{~km} \mathrm{~s}^{-1}\right)\end{array}$ & $N_{\text {pos }}^{f}$ & $\begin{array}{r}F_{\mathrm{eff}^{g}}^{g} \\
(\%)\end{array}$ & $\begin{array}{r}B_{\mathrm{eff}^{g}}^{g} \\
(\%)\end{array}$ & $\begin{array}{c}T_{\text {sys }}{ }^{h} \\
(\mathrm{~K})\end{array}$ & $\begin{array}{l}\mathrm{rms}^{i} \\
(\mathrm{mK})\end{array}$ \\
\hline & 19560.3541 & 1.5 & 27.7 & & & 76 & 0.104 & 13 & 95 & 75 & $232-240$ & $136-195$ \\
\hline $2-1$ & & 80 & & & & 76 & & 13 & & 7 & & \\
\hline & 55 & 2.8 & & & & 76 & & 5 & 95 & 7 & & \\
\hline${ }^{13} \mathrm{CO}^{+} 3-2$ & & 9.7 & & & & 76 & & 13 & 95 & 7 & & \\
\hline $\mathrm{HCO}^{+} 3-2$ & 267557.6259 & 1.1 & 22.8 & APEX-2 SSB & FFTS1 & 122 & 0.137 & 13 & 95 & 74 & $200-306$ & $120-152$ \\
\hline $\mathrm{H}^{13} \mathrm{CO}^{+} 4-3$ & 346998.3440 & 11.9 & 17.5 & FLASH345 2SB & & 76 & 0.066 & 5 & 95 & 73 & $280-305$ & $48-53$ \\
\hline $\mathrm{CO} 4-3$ & 461040.7682 & 0.5 & 13.2 & FLASH460 DSB & AFFTS & 183 & 0.119 & 5 & 95 & 60 & $1083-1288$ & $234-360$ \\
\hline
\end{tabular}

Notes. ${ }^{(a)}$ Rest frequency taken from the CDMS catalogue. The frequencies given here for the $\mathrm{H}^{13} \mathrm{CO}^{+} 3-2$ and $4-3$ transitions do not account for their hyperfine structure. See the CDMS catalogue for the specific frequencies corresponding to each hyperfine structure component of the transitions. ${ }^{(b)}$ Frequency uncertainty taken from the CDMS catalogue. ${ }^{(c)}$ Angular resolution. ${ }^{(d)}$ Channel spacing in frequency. ${ }^{(e)}$ Channel spacing in velocity. ${ }^{(f)}$ Number of observed positions. ${ }^{(g)}$ Forward and main-beam efficiencies. ${ }^{(h)}$ System temperature. ${ }^{(i)}$ rms sensitivity in $T_{\mathrm{a}}^{\star}$ scale.

Table 3. Parameters of Mopra observations.

\begin{tabular}{|c|c|c|c|c|c|c|c|c|c|}
\hline Transition & $\begin{array}{c}f^{a} \\
(\mathrm{MHz}) \\
\end{array}$ & $\begin{array}{l}\sigma_{v}^{b} \\
(\mathrm{kHz})\end{array}$ & $\begin{array}{c}H P B W^{c} \\
(")\end{array}$ & $\begin{array}{l}\delta f^{d} \\
(\mathrm{kHz})\end{array}$ & $\begin{array}{c}\delta V^{e} \\
\left(\mathrm{~km} \mathrm{~s}^{-1}\right)\end{array}$ & $N_{\text {pos }}^{f}$ & $\begin{array}{r}B_{\mathrm{eff}^{g}} \\
(\%) \\
\end{array}$ & $\begin{array}{c}T_{\text {sys }}{ }^{h} \\
(\mathrm{~K}) \\
\end{array}$ & $\begin{array}{r}\mathrm{rms}^{i} \\
(\mathrm{mK}) \\
\end{array}$ \\
\hline c- $-\mathrm{C}_{3} \mathrm{H}_{2} 3_{2,2}-3_{1,3}$ & 84727.6909 & 3.4 & 40.6 & 34 & 0.12 & 1 & 34 & 185 & 18 \\
\hline $\mathrm{HC}^{18} \mathrm{O}^{+} 1-0$ & 85162.2231 & 4.8 & 40.4 & 34 & 0.12 & 1 & 34 & 185 & 18 \\
\hline $\mathrm{H}^{13} \mathrm{CO}^{+} 1_{2,2}-0_{1,1}{ }^{j}$ & 86754.3004 & 3.9 & 39.7 & 34 & 0.12 & 1 & 34 & 165 & 17 \\
\hline $\mathrm{HN}^{13} \mathrm{C}_{1,3,3}-0_{1,2,2}{ }^{j}$ & 87090.8297 & 3.8 & 39.5 & 34 & 0.12 & 1 & 34 & 165 & 14 \\
\hline $\mathrm{HNCO} 4_{0,4,5}-3_{0,3,4}{ }^{j}$ & 87925.2178 & 0.3 & 39.2 & 34 & 0.11 & 1 & 34 & 164 & 16 \\
\hline $\mathrm{HCO}^{+} 1-0$ & 89188.5247 & 4.1 & 38.6 & 34 & 0.11 & 1 & 34 & 147 & 17 \\
\hline $\mathrm{HC}_{3} \mathrm{~N} 10_{11}-9_{10}^{j}$ & 90979.0024 & 1.0 & 37.9 & 34 & 0.11 & 9 & 34 & $208-230$ & $17-32$ \\
\hline${ }^{13} \mathrm{CS} 2-1$ & 92494.3080 & 50.0 & 37.2 & 34 & 0.11 & 9 & 34 & $208-230$ & $17-29$ \\
\hline $\mathrm{N}_{2} \mathrm{H}^{+} 1_{2,3}-0_{1,2}^{j}$ & 93173.7642 & 2.4 & 37.0 & 34 & 0.11 & 9 & 34 & $213-233$ & $19-31$ \\
\hline $\mathrm{C}^{34} \mathrm{~S} 2-1$ & 96412.9495 & 2.2 & 35.7 & 34 & 0.10 & 9 & 34 & $216-235$ & $19-35$ \\
\hline $\mathrm{CH}_{3} \mathrm{OH}-E 2_{1,2}-1_{1,1}$ & 96739.362 & 5.0 & 35.6 & 34 & 0.10 & 9 & 34 & $229-251$ & $20-33$ \\
\hline $\mathrm{CH}_{3} \mathrm{OH}-\mathrm{A} 2_{0,2}-1_{0,1}$ & 96741.375 & 5.0 & 35.6 & 34 & 0.10 & 9 & 34 & $229-251$ & $20-33$ \\
\hline $\mathrm{CH}_{3} \mathrm{OH}-E 2_{0,2}-1_{0,1}$ & 96744.550 & 5.0 & 35.6 & 34 & 0.10 & 9 & 34 & $229-251$ & $20-33$ \\
\hline$C^{33} S 2-1$ & 97172.0639 & 0.2 & 35.4 & 34 & 0.10 & 9 & 34 & $229-251$ & $20-34$ \\
\hline CS 2-1 & 97980.9533 & 2.3 & 35.2 & 34 & 0.10 & 9 & 34 & $229-251$ & $20-32$ \\
\hline
\end{tabular}

Notes. ${ }^{(a)}$ Rest frequency taken from the CDMS catalogue. ${ }^{(b)}$ Frequency uncertainty. ${ }^{(c)}$ Angular resolution. ${ }^{(d)}$ Channel spacing in frequency. ${ }^{(e)}$ Channel spacing in velocity. ${ }^{(f)}$ Number of observed positions. ${ }^{(g)}$ Main beam efficiency. ${ }^{(h)}$ Range of system temperature. ${ }^{(i)}$ rms sensitivity in $T_{a}^{\star}$ scale. ${ }^{(j)}$ Transition with hyperfine structure.

\section{5. $\mathrm{CO} 3-2$ data}

Cha-MMS1 was observed in CO 3-2 in 2005 with the APEX telescope, and the data were presented in Belloche et al. (2006). We used the CO 3-2 data along the direction perpendicular to the filament in conjuction with the other $\mathrm{CO}$ transitions and isotopologues when modelling the spectra in Sect. 4.

\section{Results}

\subsection{Internal luminosity derivation}

We performed aperture photometry on MIPS1 $24 \mu \mathrm{m}$ and MIPS2 $70 \mu \mathrm{m}$ Spitzer data, and derived flux densities for the dense core Cha-MMS1. We used the IDL procedure aper.pro ${ }^{6}$ with the following aperture and background inner and outer radii: $16^{\prime \prime}\left(18^{\prime \prime}-39^{\prime \prime}\right)$ and $13^{\prime \prime}\left(20^{\prime \prime}-32^{\prime \prime}\right)$ for the $70 \mu \mathrm{m}$ and $24 \mu \mathrm{m}$ data, respectively. Fine-scale aperture corrections of 2.16 for MIPS1 and 1.17 for MIPS2 were taken from the

\footnotetext{
6 From the IDL Astronomy User's Library (http://idlastro. gsfc. nasa.gov/contents.html).
}

MIPS Instrument Handbook ${ }^{7}$. The flux densities derived before and after correction are given in Table 4. In the following, we use the average value of the two independent, aperture-corrected $70 \mu \mathrm{m}$ flux density measurements to estimate the internal luminosity of Cha-MMS1. We determine the internal luminosity of Cha-MMS1 with two methods.

\subsubsection{Method 1}

Dunham et al. (2008) calculated the internal luminosity of lowluminosity protostars based on a parametric model consisting of a protostellar envelope, a disk, and an outflow cone, coupled to a $2 \mathrm{D}$ radiative transfer code. They derived the following empirical relation between the internal luminosity of a protostar and its observed $70 \mu \mathrm{m}$ flux:

$L_{\text {int }}=3.3 \times 10^{8} F_{70}^{0.94} L_{\odot}$,

where $\mathrm{F}_{70}$ is normalised to $140 \mathrm{pc}$ and is in cgs units $\left(\mathrm{cm}^{-2} \mathrm{~s}^{-1}\right)$. With this equation, we derive an internal luminosity of $0.025 \pm 0.003 L_{\odot}$ after correction for the distance of Cha-MMS1.

7 See http://irsa.ipac.caltech.edu/data/SPITZER/docs/ mips/mipsinstrumenthandbook/1/ 
Table 4. Flux densities of Cha-MMS1 from aperture photometry.

\begin{tabular}{|c|c|c|c|c|}
\hline Instrument & $\begin{array}{c}\lambda^{a} \\
(\mu \mathrm{m})\end{array}$ & AORkey $^{b}$ & $\begin{array}{l}F_{\lambda}^{c} \\
(\mathrm{mJy})\end{array}$ & $\begin{array}{c}F_{\lambda}^{\text {corrd }} \\
(\mathrm{mJy})\end{array}$ \\
\hline MIPS2 & 70 & $\begin{array}{c}19978496 \\
3962112 \\
\text { average }\end{array}$ & $\begin{array}{l}139 \pm 32 \\
184 \pm 25\end{array}$ & $\begin{array}{l}300 \pm 70 \\
397 \pm 54 \\
349 \pm 44\end{array}$ \\
\hline MIPS1 & 24 & $\begin{array}{c}19978496 \\
3962112 \\
19979264 \\
\text { average }\end{array}$ & $\begin{array}{l}2.82 \pm 0.64 \\
2.32 \pm 0.60 \\
2.50 \pm 0.63\end{array}$ & $\begin{array}{l}3.3 \pm 0.7 \\
2.7 \pm 0.8 \\
2.9 \pm 0.7 \\
3.0 \pm 0.4\end{array}$ \\
\hline
\end{tabular}

Notes. ${ }^{(a)}$ Wavelength. ${ }^{(b)}$ AORkey of Spitzer observations. ${ }^{(c)}$ Flux density from aperture photometry. ${ }^{(d)}$ Flux density after fine-scale correction.

\subsubsection{Method 2}

Commerçon et al. (2012) have recently presented the evolution of the $24 \mu \mathrm{m}$ and $70 \mu \mathrm{m}$ flux densities in the course of the first core lifetime, as well as the time evolution of the FHSC internal luminosity via 3D radiation-magnetohydrodynamic (hereafter, RMHD) simulations of a $1 M_{\odot}$ dense core collapse. A 3D RMHD simulation for the case of a $5 M_{\odot}$ dense core collapse was also computed (see Sect. 5.4.2). Both models have a strong initial magnetisation level (MU2 model; Commerçon et al. 2012).

We looked for a correspondence between Cha-MMS1's $24 \mu \mathrm{m}$ and $70 \mu \mathrm{m}$ flux densities and the model flux density predictions of FHSC obtained for the $1 M_{\odot}$ and $5 M_{\odot}$ dense cores. In the case of the $1 M_{\odot}$ model, we find consistent flux densities within a factor of $\sim 2$ for inclinations to the line-of-sight $45^{\circ}<i<60^{\circ}$, and a first core age of $850 \mathrm{yr}$. In this case, the internal luminosity prediction is $\sim 0.08 L_{\odot}-0.13 L_{\odot}$, at least three times higher than the internal luminosity derived using the relation by Dunham et al. (2008).

In the $5 M_{\odot}$ case at inclinations $30^{\circ}<i<45^{\circ}$ and for a first core age of $\sim 2680 \mathrm{yr}$, the observed and predicted $24 \mu \mathrm{m}$ flux densities are consistent within a factor of $\sim 2.5$, and we thus obtain an internal luminosity estimate of $\sim 0.13 L_{\odot}-0.18 L_{\odot}$.

In the framework of this MHD model, the overall range is $\sim 0.08 L_{\odot}-0.18 L_{\odot}$ for inclinations of $30^{\circ} \leq i<60^{\circ}$. The internal luminosity derived from the empirical relation of Dunham et al. (2008) is therefore lower by a factor of $\sim 3-7$ compared to the predictions of 3D RMHD simulations.

We adopt an internal luminosity of $\sim 0.1 L_{\odot}$ as an approximation, which is within the luminosity range we derived based on the RMHD simulations. We use this value for the radiative transfer modelling that follows in Sect. 4. Even if we were to adopt the upper limit of $0.18 L_{\odot}$, the temperature profile of the inner envelope would not significantly change (Eq. (7), Sect. 4.1).

\subsection{Spectra towards Cha-MMS1}

Figures 2 and 3 show the spectra of the transitions observed with APEX and Mopra towards the central position of Cha-MMS1. Apart from ${ }^{13} \mathrm{CS} 2-1$ and $\mathrm{C}^{33} \mathrm{~S} 2-1$ for which we can only draw upper limits, most transitions are detected. Tables 5 to 9 give the centroid velocities derived for these transitions after performing Gaussian or hyperfine-structure fits in CLASS to the observed spectra ("GAUSS" and "HFS" methods). Only the spectra that have either a Gaussian shape or a well-defined hyperfine structure and no self-absorption features can be fitted in this way. Tables 5 to 9 list the systemic velocities of groups of transitions observed at the same offset positions (see Fig 1). The centroid velocities of transitions that were only observed at the central position of the core are given in Table 6 .

\subsubsection{Issues with the systemic velocity}

It is apparent from Fig. 3 that all the APEX spectra are redshifted by $0.1 \mathrm{~km} \mathrm{~s}^{-1}$ compared to the systemic velocity derived from a hyperfine-structure fit to the $\mathrm{N}_{2} \mathrm{H}^{+} 1-0$ multiplet observed with Mopra. There is therefore a problem with one of the two datasets. As the shift is seen for both the high and low-density APEX tracers (e.g., $\mathrm{H}^{13} \mathrm{CO}^{+} 4-3$ and $\mathrm{C}^{18} \mathrm{O} 2-1$ ), it is not likely to be an intrinsic characteristic of the source, but rather an instrumental effect.

We compared the spectra of the calibration source IRAM 04191, observed with APEX, Mopra, and with the IRAM 30-m telescope, to pinpoint the source of the systemic velocity inconsistency. In addition, because we have Mopra observations of the central position of Cha-MMS1 from both 2010 and 2012 (as part of a survey targeting starless cores in Cha I and III, Tsitali et al. in prep.), we compared them to the 2011 data that we present in this paper. The $\mathrm{N}_{2} \mathrm{H}^{+} 1-0$ IRAM 04191 spectra are consistent with each other, and consequently, we cannot draw any conclusions about the velocity shift. However, the $\mathrm{N}_{2} \mathrm{H}^{+} 1-0$ central spectrum of Cha-MMS1 observed with Mopra in 2010 and 2012 gives a velocity estimate consistent with the APEX data, i.e. $4.4 \mathrm{kms}^{-1}$, but inconsistent with the 2011 Mopra data, i.e. $4.3 \mathrm{kms}^{-1}$. We therefore assume that the Mopra 2010, 2012, and APEX 2011 datasets are correct and apply a correction of $0.1 \mathrm{kms}^{-1}$ to the systemic velocity derived from the 2011 Mopra $\mathrm{N}_{2} \mathrm{H}^{+} 1-0$ transition whenever we use it along with the 2011 APEX spectra. We explicitly mention it in the text whenever this correction is applied.

\subsection{Rotation}

We produced position-velocity $(P-V)$ diagrams for the Mopra $\mathrm{C}^{34} \mathrm{~S} 2-1, \mathrm{HC}_{3} \mathrm{~N}$ 10-9, $\mathrm{N}_{2} \mathrm{H}^{+} 1-0, \mathrm{CH}_{3} \mathrm{OH}-A \quad 2_{0,2}-1_{0,1}$, and $\mathrm{CH}_{3} \mathrm{OH}-\mathrm{E} 2_{1,2}-1_{1,1}$ transitions and the APEX CS 5-4, $\mathrm{H}^{13} \mathrm{CO}^{+} 3-2, \mathrm{H}^{13} \mathrm{CO}^{+} 4-3, \mathrm{C}^{17} \mathrm{O} 2-1$, and $\mathrm{C}^{18} \mathrm{O} 2-1$ transitions (Figs. 4 and 5) based on the centroid velocities measured in Sect. 3.2. We performed linear fits to these $P-V$ diagrams to search for velocity gradients. The results are listed in Table 10 and shown in Figs. 4 and 5. After combining all tracers, there is no clear velocity gradient parallel to the filament $\left(\leq 2 \mathrm{~km} \mathrm{~s}^{-1} \mathrm{pc}^{-1}\right.$, see Figs. $4 \mathrm{~b}$ and $\left.5 \mathrm{~d}\right)$.

The $P-V$ diagrams for the direction perpendicular to the filament are given in Figs. $4 \mathrm{a}$ and $5 \mathrm{a}-\mathrm{c}$. There is a clear velocity gradient along this direction with an amplitude of $\sim 2 \mathrm{~km} \mathrm{~s}^{-1}-4.5 \mathrm{~km} \mathrm{~s}^{-1} \mathrm{pc}^{-1}$ up to $\sim 8000$ AU. The average velocity gradient is $\sim 3.1 \pm 0.1 \mathrm{~km} \mathrm{~s}^{-1} \mathrm{pc}^{-1}$. However, the $\mathrm{C}^{17} \mathrm{O} 2-1$ and $\mathrm{C}^{18} \mathrm{O} 2-1$ curves are significantly flatter at the inner, $r \leq 4000 \mathrm{AU}$ radii, compared to the range $4000 \mathrm{AU}-8000 \mathrm{AU}$ (Fig. 5c), with velocity gradients $\leq 2 \mathrm{~km} \mathrm{~s}^{-1} \mathrm{pc}^{-1}$. The $\mathrm{H}^{13} \mathrm{CO}^{+} 4-3$ and $\mathrm{H}^{13} \mathrm{CO}^{+} 3-2 P-V$ curves are also consistent with no gradient for the inner $\sim 4000 \mathrm{AU}$.

The $P-V$ curves of $\mathrm{C}^{17} \mathrm{O} 2-1$ and $\mathrm{C}^{18} \mathrm{O} 2-1$ depart from a straight line for radii larger than $\sim 8000 \mathrm{AU}$. The weighted average velocity gradient for the two transitions between the two outermost positions (at $\pm 12500 \mathrm{AU}$ ) is $\sim 1.5 \pm 0.2 \mathrm{~km} \mathrm{~s}^{-1} \mathrm{pc}^{-1}$, i.e. smaller by a factor of $\sim 2$ than within 8000 AU. Such an "S" shape was reported by Belloche et al. (2002) for the Class 0 protostar IRAM 04191 and was interpreted as an indication of 
A. E. Tsitali et al.: The dynamical state of the first hydrostatic core candidate Chamaeleon-MMS1

Table 5. Mopra centroid velocities at positions perpendicular and parallel to the filament.

\begin{tabular}{lcccccccc}
\hline \hline Position $^{a}$ & $\begin{array}{c}\Delta \alpha^{b} \\
(")\end{array}$ & $\begin{array}{c}\Delta \delta^{b} \\
(\prime)\end{array}$ & $\begin{array}{c}\text { Spacing }^{c} \\
\left({ }^{\prime \prime}\right)\end{array}$ & $\mathrm{C}^{34} \mathrm{~S} 2-1$ & $\mathrm{HC}_{3} \mathrm{~N} \mathrm{10-9}$ & $V_{\mathrm{LSR}_{2}{ }^{d}\left(\mathrm{~km} \mathrm{~s}^{+1}\right)} 1-0^{e}$ & $\mathrm{CH}_{3} \mathrm{OH}-A 2_{0,2}-1_{0,1}$ & $\mathrm{CH}_{3} \mathrm{OH}-E 2_{1,2}-1_{1,1}$ \\
\hline $\mathrm{P} 1$ & 0 & 0 & 0 & $4.33 \pm 0.02$ & $4.27 \pm 0.004$ & $4.299 \pm 0.002$ & $4.29 \pm 0.01$ & $4.29 \pm 0.01$ \\
$\mathrm{P}_{d} 2$ & -10.0 & 14.3 & 17.5 & $4.30 \pm 0.02$ & $4.29 \pm 0.004$ & $4.279 \pm 0.002$ & $4.29 \pm 0.01$ & $4.28 \pm 0.01$ \\
$\mathrm{P}_{d} 3$ & -20.1 & 28.7 & 35.0 & $4.26 \pm 0.02$ & $4.23 \pm 0.004$ & $4.339 \pm 0.002$ & $4.25 \pm 0.01$ & $4.25 \pm 0.01$ \\
$\mathrm{P}_{d} 4$ & -30.1 & 43.0 & 52.5 & $4.21 \pm 0.02$ & $4.12 \pm 0.004$ & $4.168 \pm 0.002$ & $4.21 \pm 0.01$ & $4.21 \pm 0.01$ \\
$\mathrm{P}_{d} 5$ & 10.0 & -14.3 & 17.5 & $4.35 \pm 0.02$ & $4.34 \pm 0.004$ & $4.346 \pm 0.003$ & $4.33 \pm 0.01$ & $4.34 \pm 0.01$ \\
$\mathrm{P}_{d} 6$ & 20.1 & -28.7 & 35.0 & $4.39 \pm 0.02$ & $4.41 \pm 0.004$ & $4.403 \pm 0.004$ & $4.37 \pm 0.01$ & $4.36 \pm 0.01$ \\
$\mathrm{P}_{d} 7$ & 30.1 & -43.0 & 52.5 & $4.42 \pm 0.02$ & $4.47 \pm 0.006$ & $4.435 \pm 0.005$ & $4.41 \pm 0.01$ & $4.38 \pm 0.01$ \\
$\mathrm{P}_{d} 8$ & 20.1 & 28.7 & 35.0 & $4.39 \pm 0.02$ & $4.33 \pm 0.006$ & $4.294 \pm 0.004$ & $4.29 \pm 0.02$ & $4.35 \pm 0.02$ \\
$\mathrm{P}_{d} 9$ & -20.1 & -28.7 & 35.0 & $4.35 \pm 0.02$ & $4.36 \pm 0.001$ & $4.286 \pm 0.004$ & $4.36 \pm 0.01$ & $4.35 \pm 0.01$ \\
\hline
\end{tabular}

Notes. ${ }^{(a)}$ These positions are shown in Fig. 1f. ${ }^{(b)}$ J2000 equatorial offset relative to the central position P1. ${ }^{(c)}$ Angular distance to P1. ${ }^{(d)}$ The correction of $0.1 \mathrm{~km} \mathrm{~s}^{-1}$ discussed in Sect. 3.2.1 has not been applied. ${ }^{(e)} \mathrm{HC}_{3} \mathrm{~N} 10-9$ and $\mathrm{N}_{2} \mathrm{H}^{+} 1-0$ both have a hyperfine structure and were therefore fitted using the method "HFS" with CLASS.

Table 10. Velocity gradients perpendicular and parallel to the filament.

\begin{tabular}{lcccc}
\hline \hline & \multicolumn{2}{c}{$\begin{array}{c}\text { Perpendicular } \\
\text { to filament }\end{array}$} & \multicolumn{2}{c}{$\begin{array}{c}\text { Parallel } \\
\text { to filament }\end{array}$} \\
$\begin{array}{c}\nabla \boldsymbol{v}^{a} \\
\left(\mathrm{~km} \mathrm{~s}^{-1} \mathrm{pc}^{-1}\right)\end{array}$ & $\begin{array}{c}\text { Extent } \\
(\mathrm{AU})\end{array}$ & $\begin{array}{c}\nabla \boldsymbol{v}^{a} \\
\left(\mathrm{~km} \mathrm{~s}^{-1} \mathrm{pc}^{-1}\right)\end{array}$ & $\begin{array}{c}\text { Extent } \\
(\mathrm{AU})\end{array}$ \\
\hline $\mathrm{C}^{34} \mathrm{~S} 2-1$ & $2.6 \pm 0.1$ & 15750 & $0.6 \pm 1.0$ & 5250 \\
$\mathrm{HC}_{3} \mathrm{~N} 10-9$ & $4.0 \pm 0.5$ & 15750 & $-1.6 \pm 1.5$ & 5250 \\
$\mathrm{~N}_{2} \mathrm{H}^{+} 1-0$ & $3.4 \pm 0.2$ & 15750 & $0.1 \pm 0.1$ & 5250 \\
$\mathrm{CH}_{3} \mathrm{OH}-A 2_{0,2}-1_{0,1}$ & $2.4 \pm 0.2$ & 15750 & $-2.0 \pm 0.7$ & 5250 \\
$\mathrm{CH}_{3} \mathrm{OH}-E 2_{1,2}-1_{1,1}$ & $2.2 \pm 0.2$ & 15750 & $-1.5 \pm 1.4$ & 5250 \\
$\mathrm{H}^{13} \mathrm{CO}^{+} 3-2$ & $0.6 \pm 0.6$ & 8550 & $-1.9 \pm 0.8$ & 10260 \\
$\mathrm{H}^{13} \mathrm{CO}^{+} 4-3$ & $0.6 \pm 1.1$ & 5130 & - & - \\
$\mathrm{C}^{17} \mathrm{O} 2-1$ & $3.6 \pm 0.5$ & 16800 & - & - \\
$\mathrm{C}^{17} \mathrm{O} 2-1^{c}$ & $1.3 \pm 0.7$ & 6300 & - & - \\
$\mathrm{C}^{18} \mathrm{O} 2-1$ & $3.2 \pm 0.5$ & 16800 & - & - \\
$\mathrm{C}^{18} \mathrm{O} 2-1^{c}$ & $1.7 \pm 0.2$ & 6300 & - & - \\
\hline
\end{tabular}

Notes. ${ }^{(a)}$ The velocity gradients were estimated from linear fits to the position-velocity curves in Figs. 4 and 5. ${ }^{(b)}$ Total extent over which a linear fit to the data was performed. ${ }^{(c)}$ Gradients corresponding to the fits limited to the inner parts in Fig. $5 \mathrm{c}$.

differential rotation in the envelope beyond a certain radius. If the velocity gradient of Cha-MMS1 perpendicular to the filament is due to rotation, then the bulk of the envelope is roughly in solid-body rotation between $\sim 4000 \mathrm{AU}$ and $8000 \mathrm{AU}$, and the outermost parts of the filament are rotating more slowly. After correcting for an inclination of $60^{\circ}-30^{\circ}$ (Sect. 3.1), the average angular velocity for radii between $4000 \mathrm{AU}$ and $8000 \mathrm{AU}$ is $\Omega \sim 3.6 \mathrm{~km} \mathrm{~s}^{-1} \mathrm{pc}^{-1}-6.2 \mathrm{~km} \mathrm{~s}^{-1} \mathrm{pc}^{-1}$. At $12500 \mathrm{AU}$, and for the same inclinations, we obtain $\Omega \sim 1.8 \mathrm{~km} \mathrm{~s}^{-1}$ $\mathrm{pc}^{-1}-3.0 \mathrm{~km} \mathrm{~s}^{-1} \mathrm{pc}^{-1}$.

The $P-V$ diagram of CS 5-4 (Fig. 5a) shows a centrally peaked shape. The profile is not well resolved, but the centroid velocity at the central position is significantly higher than at one beam spacing on each side. This velocity shift is because there is an excess of redshifted emission toward the central position that is not reproduced by our radiative transfer modelling (see Sect. 4 and Fig. 10). This issue is discussed in Sect. 5.2.

\subsection{Turbulence}

The spatial variation of the non-thermal velocity dispersion is shown in Fig. 6 for several transitions. The FWHM linewidths were estimated using the "GAUSS" and "HFS" fitting methods in CLASS (see Table 11), as mentioned in Sect. 3.2. We computed the thermal velocity dispersion of each molecule as

$\sigma_{\text {th }}=\sqrt{\frac{k_{\mathrm{B}} T}{\mu_{\mathrm{M}} m_{\mathrm{H}}}}$,

where $\mu_{\mathrm{M}}$ is the molecular weight of the molecule, $k_{\mathrm{B}}$ the Boltzmann's constant, $m_{\mathrm{H}}$ the hydrogen mass, and $T$ the kinetic temperature that we assume to be $9 \mathrm{~K}$ (see Sect. 4). The nonthermal linewidths are computed as

$\sigma_{\text {nth }}=\sqrt{\sigma_{\mathrm{obs}^{2}}{ }^{2} \sigma_{\mathrm{th}^{2}}}$

while their respective uncertainties, denoted as $\delta \sigma$, are calculated using

$\delta \sigma_{\mathrm{nth}}=\sqrt{\left(\frac{\partial \sigma_{\mathrm{nth}}}{\partial \sigma_{\mathrm{observed}}}\right)^{2} \delta \sigma_{\mathrm{obs}}{ }^{2}+\left(\frac{\partial \sigma_{\mathrm{nth}}}{\partial \sigma_{\mathrm{th}}}\right)^{2} \delta \sigma_{\mathrm{th}}{ }^{2}}$.

If we assume that $\delta \sigma_{\text {th }}=0$, the above relation gives

$\delta \sigma_{\mathrm{nth}}=\frac{\sigma_{\mathrm{obs}} \times \delta \sigma_{\mathrm{obs}}}{\sigma_{\mathrm{nth}}}$.

We compare the non-thermal velocity dispersion to the thermal dispersion of the mean particle, with $\mu=2.37$ :

$\sigma_{\text {th,mean }}=\sqrt{\frac{k_{B} T}{\mu m_{H}}}$.

For $T=9 \mathrm{~K}, \sigma_{\text {th,mean }} \sim 0.18 \mathrm{~km} \mathrm{~s}^{-1}$, or $F W H M_{\text {th,mean }} \sim$ $0.42 \mathrm{~km} \mathrm{~s}^{-1}$. Table 11 lists the observed linewidths and the derived thermal and non-thermal velocity dispersions for all transitions that have a low optical depth.

From Table 11 we infer that the non-thermal velocity dispersion is comparable to the mean thermal velocity dispersion. Therefore, there is almost an equipartition between thermal and non-thermal motions, provided that our asumption of $T=9 \mathrm{~K}$ is valid.

The non-thermal dispersion does not vary significantly along or across the filament (Figs. 6 and 7). One exception is, however, CS 5-4. Its non-thermal linewidth peaks at the centre and decreases at the outer parts. This is due to the excess redshifted emission observed at the central position (see Sect. 3.3, and Sects. 4 and 5.2 for further discussion). 

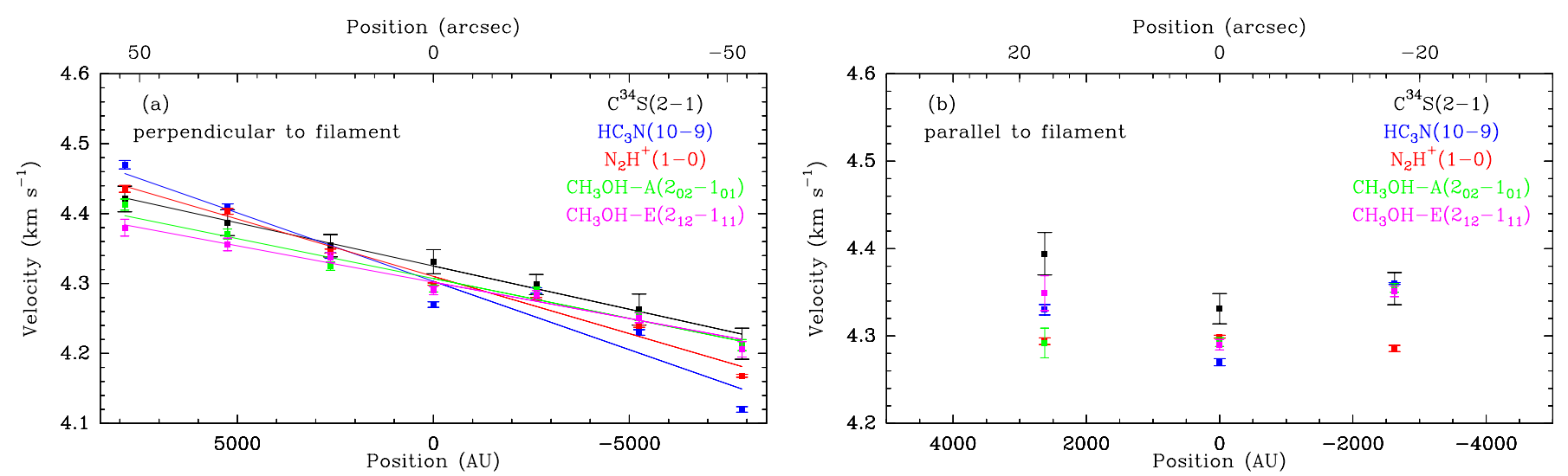

Fig. 4. Position-velocity diagrams of the Mopra $\mathrm{C}^{34} \mathrm{~S} 2-1$ (black), $\mathrm{HC}_{3} \mathrm{~N} 10-9$ (blue), $\mathrm{N}_{2} \mathrm{H}^{+} 1-0$ (red), $\mathrm{CH}_{3} \mathrm{OH}-A 2_{0,2}-1_{0,1}$ (green), and $\mathrm{CH}_{3} \mathrm{OH}-E 2_{1,2}-1_{1,1}$ (pink) transitions, a) perpendicular and b) parallel to the filament. The error bars represent standard deviations $(1 \sigma)$. Linear fits to the velocities for each transition are shown with the same colour as in panel a).
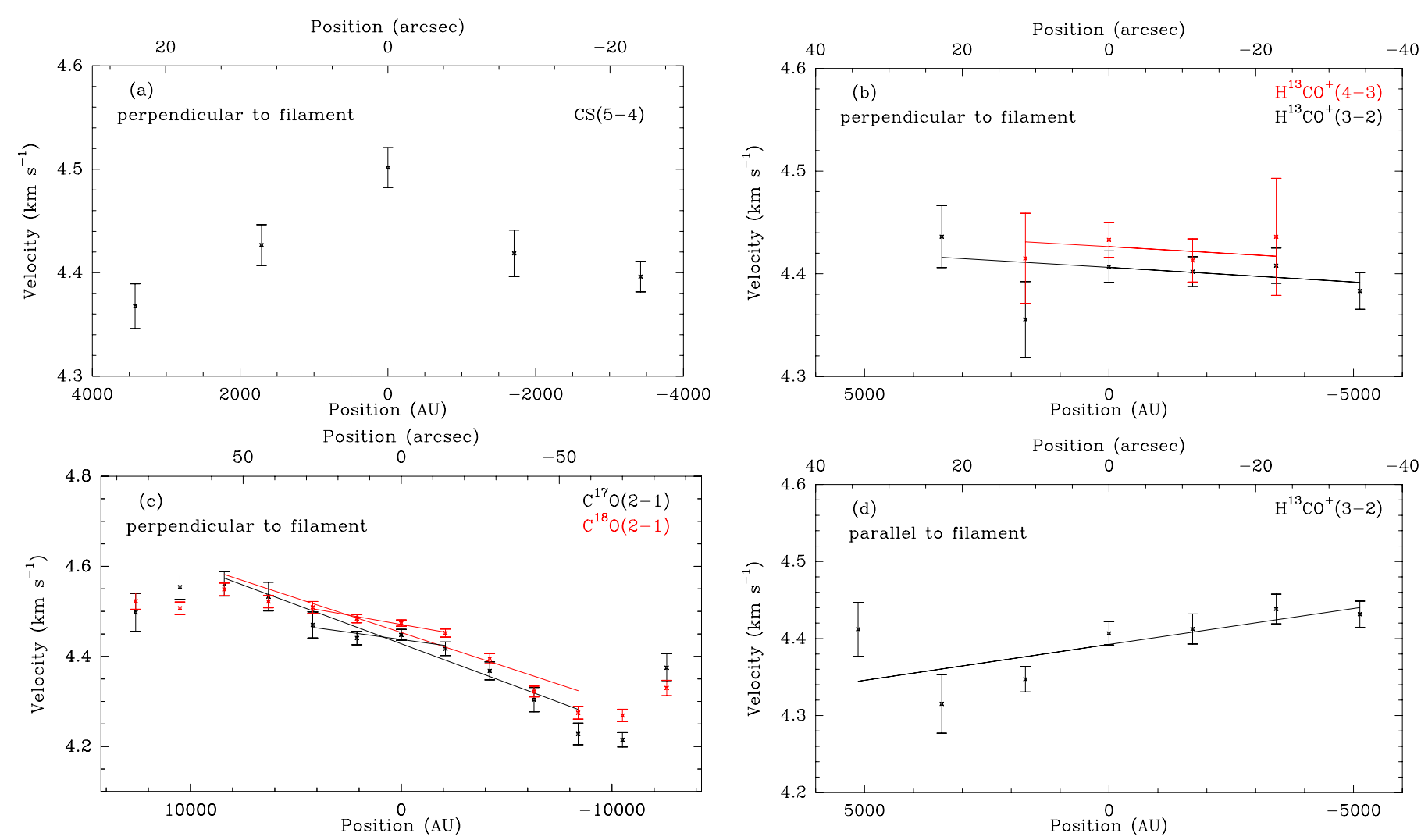

Fig. 5. Position-velocity diagrams of the APEX CS $5-4, \mathrm{H}^{13} \mathrm{CO}^{+} 3-2, \mathrm{H}^{13} \mathrm{CO}^{+} 4-3, \mathrm{C}^{17} \mathrm{O} 2-1$, and $\mathrm{C}^{18} \mathrm{O} 2-1$ transitions perpendicular a), b), c) and parallel d) to the filament. Linear fits are shown as straight lines. For $\mathrm{C}^{17} \mathrm{O} 2-1$ and $\mathrm{C}^{18} \mathrm{O} 2-1$ in c) the two outer points from either side of the curve were excluded from the fits. Fits to the innermost positions of $\mathrm{C}^{17} \mathrm{O} 2-1$ and $\mathrm{C}^{18} \mathrm{O} 2-1$ are also displayed.

Average values for the linewidths and non-thermal velocity dispersions over all positions are given in Table 12. The nonthermal dispersions, $\sigma_{\text {nth }}$, have typical values of $0.2 \mathrm{~km} \mathrm{~s}^{-1}$, comparable to the mean thermal dispersion. Uniform nonthermal dispersions on scales $\sim 0.1 \mathrm{pc}$ similar to the thermal dispersions have also been previously observed in other dense cores (e.g., Tafalla et al. 2004; Barranco \& Goodman 1998).

\subsection{Infall signature}

Cha-MMS1 exhibits the classical signature of infall in various transitions (Fig. 8). The infall signature manifests itself as a self-absorbed asymmetric, optically thick line with the blue peak stronger than the red one, and an optically thin line peaking in between these two peaks. This profile is indicative of inward motions as long as the excitation temperature increases towards the centre (e.g., Walker et al. 1986; Zhou 1992).

Figure 8 shows that the absorption dips of CS 2-1 and $\mathrm{HCO}^{+} 3-2$ are redshifted with respect to the systemic velocity. The systemic velocity was estimated by a seven-component hyperfine-structure fit to the $\mathrm{N}_{2} \mathrm{H}^{+} 1-0$ multiplet, using the HFS method in CLASS, giving a value of $4.299 \pm 0.002 \mathrm{~km} \mathrm{~s}^{-1}$. As discussed in Sect. 3.2.1, we apply a correction of $0.1 \mathrm{~km} \mathrm{~s}^{-1}$ to this systemic velocity to compare it to the APEX spectra. The 

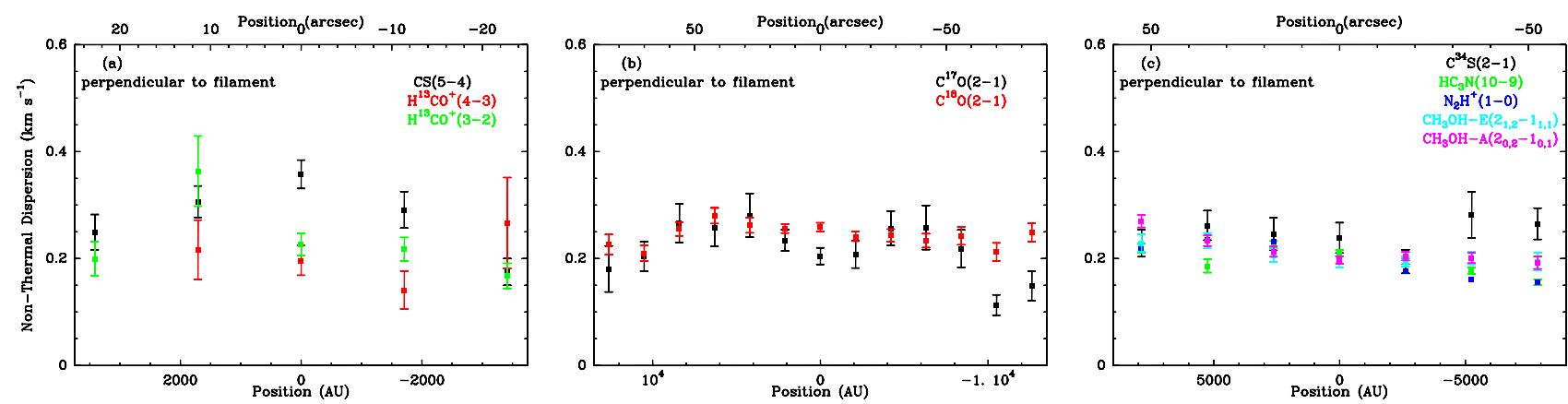

Fig. 6. Non-thermal velocity dispersion, $\sigma_{\text {nth }}$, versus position perpendicular to the filament for a) $\mathrm{CS} 5-4$ (black), $\mathrm{H}^{13} \mathrm{CO} 4-3$ (red), and $\mathrm{H}^{13} \mathrm{CO}^{+} 3-2$ (green), b) $\mathrm{C}^{17} \mathrm{O} 2-1$ and $\mathrm{C}^{18} \mathrm{O} 2-1$, all observed with the APEX telescope, and c) $\mathrm{C}^{34} \mathrm{~S} 2-1$ (black), $\mathrm{HC}_{3} \mathrm{~N} 10-9$ (green), $\mathrm{N}_{2} \mathrm{H}^{+} 1-0$ (dark blue), $\mathrm{CH}_{3} \mathrm{OH}-E 2_{1,2}-1_{1,1}$ (light blue), and $\mathrm{CH}_{3} \mathrm{OH}-A 2_{0,2}-1_{0,1}$ (pink) observed with Mopra. The errorbars are standard deviations. The thermal dispersion was calculated assuming a temperature of $T=9 \mathrm{~K}$.
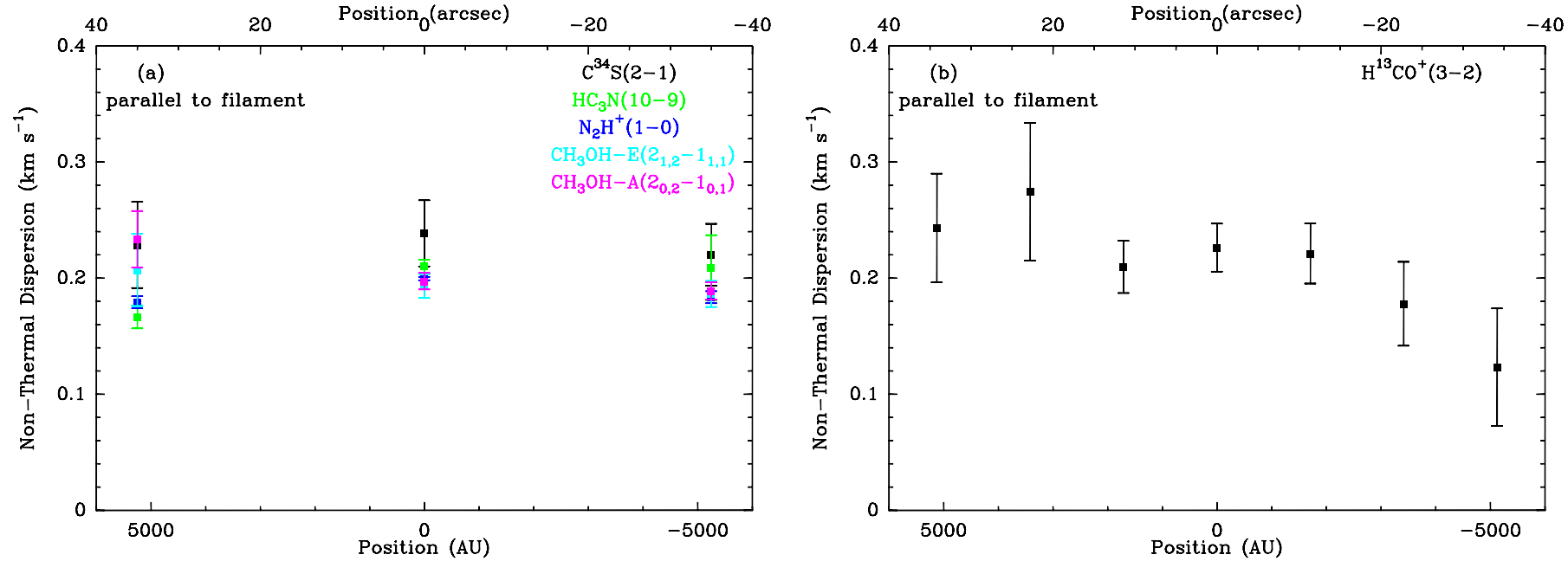

Fig. 7. Non-thermal velocity dispersion, $\sigma_{\text {nth }}$, versus position parallel to the filament for a) $\mathrm{C}^{34} \mathrm{~S} 2-1$ (black), $\mathrm{HC}_{3} \mathrm{~N} 10-9$ (green), $\mathrm{N}_{2} \mathrm{H}^{+} 1-0$ (dark blue), $\mathrm{CH}_{3} \mathrm{OH}-E 2_{1,2}-1_{1,1}$ (light blue), and $\mathrm{CH}_{3} \mathrm{OH}-A 2_{0,2}-1_{0,1}$ (pink) observed with Mopra, and b) $\mathrm{H}^{13} \mathrm{CO}^{+} 3-2$ observed with the APEX telescope. The errorbars are standard deviations. The thermal dispersion was calculated assuming a temperature of $T=9 \mathrm{~K}$.

Table 12. Linewidths and non-thermal velocity dispersions averaged over all positions.

\begin{tabular}{lccc}
\hline \hline Line & $\begin{array}{c}F W H M \\
\left(\mathrm{~km} \mathrm{~s}^{-1}\right)\end{array}$ & $\begin{array}{c}\sigma_{\text {nth }} \\
\left(\mathrm{km} \mathrm{s}^{-1}\right)\end{array}$ & $\frac{\sigma_{\text {nth }}}{\sigma_{\text {th,mean }}}$ \\
\hline $\mathrm{C}^{34} \mathrm{~S} 2-1$ & $0.57 \pm 0.009$ & $0.24 \pm 0.002$ & $1.33 \pm 0.17$ \\
$\mathrm{HC}_{3} \mathrm{~N} \mathrm{10-9}$ & $0.47 \pm 0.003$ & $0.20 \pm 0.001$ & $1.09 \pm 0.05$ \\
$\mathrm{~N}_{2} \mathrm{H}^{+} 1-0$ & $0.47 \pm 0.005$ & $0.19 \pm 0.003$ & $1.08 \pm 0.02$ \\
$\mathrm{CH}_{3} \mathrm{OH}-A 2_{0,2}-1_{0,1}$ & $0.51 \pm 0.003$ & $0.21 \pm 0.001$ & $1.18 \pm 0.06$ \\
$\mathrm{CH}_{3} \mathrm{OH}-E 2_{1,2}-1_{1,1}$ & $0.49 \pm 0.004$ & $0.20 \pm 0.001$ & $1.13 \pm 0.08$ \\
$\mathrm{H}^{13} \mathrm{CO}^{+} 3-2$ & $0.51 \pm 0.01$ & $0.21 \pm 0.004$ & $1.18 \pm 0.27$ \\
$\mathrm{H}^{13} \mathrm{CO}^{+} 4-3$ & $0.50 \pm 0.02$ & $0.20 \pm 0.005$ & $1.14 \pm 0.28$ \\
$\mathrm{C}^{17} \mathrm{O} 2-1$ & $0.53 \pm 0.01$ & $0.22 \pm 0.002$ & $1.21 \pm 0.17$ \\
$\mathrm{C}^{18} \mathrm{O} 2-1$ & $0.59 \pm 0.005$ & $0.24 \pm 0.001$ & $1.35 \pm 0.08$ \\
$\mathrm{CS} 5-4{ }^{a}$ & $0.61 \pm 0.02$ & $0.26 \pm 0.004$ & $1.42 \pm 0.17$ \\
\hline
\end{tabular}

Notes. ${ }^{(a)}$ The linewidth and non-thermal velocity dispersion of the central CS 5-4 position was not taken into account for computing the average value, owing to the spectrum's broadened shape. See Sect. 5.2 for further discussion.

self-absorption dip of the optically thick CS 2-1 transition has a velocity of $4.50 \pm 0.05 \mathrm{~km} \mathrm{~s}^{-1}$, which gives a velocity shift of $0.20 \pm 0.05 \mathrm{~km} \mathrm{~s}^{-1}$. Using the corrected value of the systemic velocity we derive a velocity shift of $0.18 \pm 0.05 \mathrm{~km} \mathrm{~s}^{-1}$ for the optically thick $\mathrm{HCO}^{+} 3-2$ line, whose absorption dip has a velocity of $4.58 \pm 0.06 \mathrm{~km} \mathrm{~s}^{-1}$. Since the self-absorption dips are produced by the outer parts of the envelope where the opacity of the $\mathrm{CS}$ and $\mathrm{HCO}^{+}$lines becomes unity, this observed redshift points to the fact that the outer layers of ChaMMS1 undergo inward motions with velocities of the order of $0.2 \mathrm{~km} \mathrm{~s}^{-1}$.

\subsection{Overview of $\mathrm{CHAMP}^{+}$data}

We probed Cha-MMS1 in the CO 6-5, CO 7-6, and ${ }^{13} \mathrm{CO} 6-5$ molecular transitions with the APEX CHAMP ${ }^{+} 2 \times 7$-pixel heterodyne receiver array in order to search for emission indicative of outflowing material. Figure 9 shows position maps of all spectra obtained for each transition. The CO 6-5 and 7-6 spectra along the filament have slightly higher intensities toward the north-east as opposed to the south-west. This could be due to contamination from the outflow of the Class I object IRAS 11051-7706 lying close to Cha-MMS1 at the north-east direction (Belloche et al. 2006), and it is further discussed in Sect. 5.2. 
A\&A 557, A98 (2013)

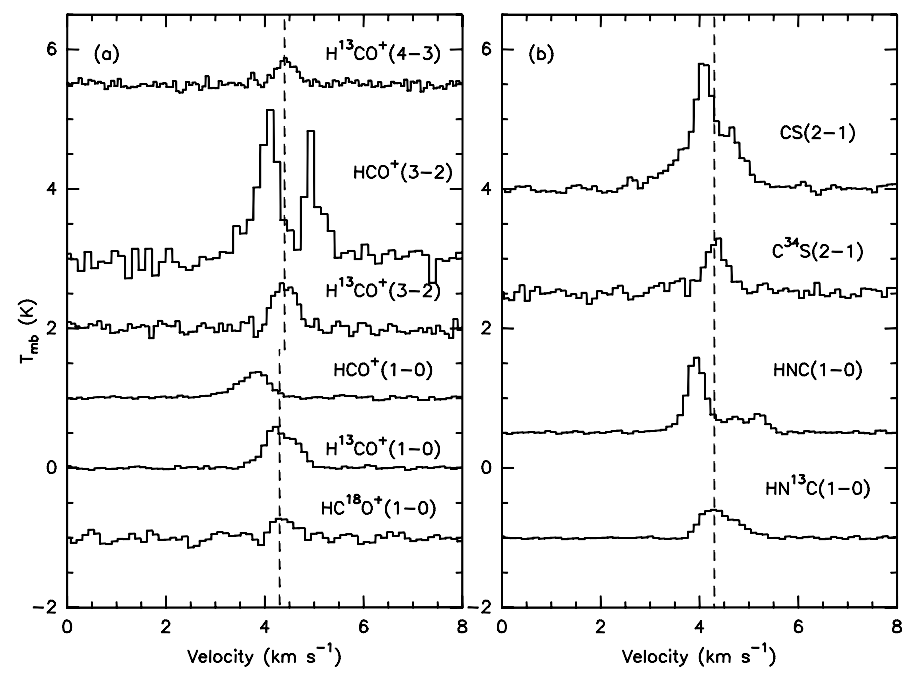

Fig. 8. Spectra obtained toward the central position of Cha-MMS1 in the optically thick $\mathrm{CS} 2-1, \mathrm{HCO}^{+} 3-2, \mathrm{HCO}^{+} 1-0$, and $\mathrm{HNC}$ 1-0 transitions and the low optical depth $\mathrm{C}^{34} \mathrm{~S} 2-1, \mathrm{H}^{13} \mathrm{CO}^{+} 1-0$, $\mathrm{H}^{13} \mathrm{CO}^{+} 3-2, \mathrm{H}^{13} \mathrm{CO}^{+} 4-3, \mathrm{HC}^{18} \mathrm{O}^{+} 1-0$, and $\mathrm{HN}^{13} \mathrm{C} 1-0$ transitions. The dashed line corresponds to the systemic velocity derived from a seven-component hyperfine fit to the $\mathrm{N}_{2} \mathrm{H}^{+} 1-0$ multiplet $\left(4.3 \mathrm{~km} \mathrm{~s}^{-1}\right)$. It is corrected to $4.4 \mathrm{~km} \mathrm{~s}^{-1}$ for the APEX transitions $\mathrm{HCO}^{+} 3-2$, $\mathrm{H}^{13} \mathrm{CO}^{+} 3-2$, and $\mathrm{H}^{13} \mathrm{CO}^{+} 4-3$ (see Sect. 3.2.1).

\section{Radiative transfer modelling}

We used the Monte Carlo radiative transfer code MAPYSO (Blinder 1997; Belloche et al. 2002), which assumes spherical symmetry, to derive kinematic constraints on the dense core Cha-MMS1 by modelling the observed spectra. We modelled the emission of three sets of molecules, namely $\mathrm{CS}, \mathrm{HCO}^{+}$, and $\mathrm{CO}$ with their respective isotopologues.

We model all molecular transitions with the same density, temperature, and turbulence distributions, as described below. These distributions are shown in Figs. 11a, c, and e, respectively. Given the shape of the continuum emission, we assume that ChaMMS1 is embedded in a filament and that the physical structure perpendicular to the filament in the plane of the sky is similar to the structure along the line-of-sight. We thus model the spectra taken along the direction perpendicular to the filament in addition to the central spectra. We perform the fit optimisation by eye, by focussing on three main features of the spectra: the peak temperature, the position of the self-absorption dip (when there is one), and the linewidth of each spectrum.

\subsection{Input parameters}

\subsubsection{Temperature profile}

Using the internal luminosity derived in Sect. 3.1 we can constrain the inner dust temperature profile of the source, which we assume is dominated by the central heating. Following Terebey et al. (1993) and Motte \& André (2001), we assume that in the inner part the dust temperature behaves as

$T_{\text {dust }}(r)=38 \mathrm{~K} \times\left(\frac{r}{100 \mathrm{AU}}\right)^{-q} \times\left(\frac{L_{\mathrm{int}}}{1 L_{\odot}}\right)^{q / 2}$,

with

$q=\frac{2}{4+\beta}$
The constant $\beta$ depends on the dust properties and the values 2 , 1.5 , and 1 are often used for molecular clouds, protostellar envelopes, and protostellar disks, respectively. Because this source is possibly at a very early evolutionary stage, perhaps before the protostellar phase, we adopt an intermediate value between the first two, 1.85, which gives $q \sim 0.34$.

We assume that the gas and dust are coupled well for densities above $\sim 10^{5} \mathrm{~cm}^{-3}$ (Lesaffre et al. 2005), and we use Eq. (7) to define the kinetic temperature in the inner part. We assume a uniform temperature in the outer parts. We find from the modelling that a uniform kinetic temperature of $9 \mathrm{~K}$ produces spectra in agreement with the observations. More specifically, the optically thick CS 2-1 line sets the major constraints on the outer kinetic temperature, since this transition shows a strong asymmetry in the blue-red peak strengths (infall signature) with the blue peak stronger by about a factor of $\sim 2$. Its absorption dip and asymmetry require uniform outer temperatures to be well fitted. The radius at which the temperature drops to $9 \mathrm{~K}$ is fixed by the central heating (Eq. (7)).

\subsubsection{Density profile}

We use a power-law density profile with an external radius of $60000 \mathrm{AU}$. We adopt a spherically symmetric $r^{-2}$ density profile for the envelope, as is expected from models of spherical gravitational collapse of non-singular isothermal spheres during the core formation either without the effect of magnetic fields (e.g., Masunaga et al. 1998; Foster \& Chevalier 1993; Bodenheimer \& Sweigart 1968; Larson 1969; Penston 1969) or from models of axisymmetric, isothermal cloud contraction with magnetic fields (e.g., Tassis \& Mouschovias 2007; Fiedler \& Mouschovias 1993). Very recently, a 3D radiation hydrodynamic simulation of the collapse of an axisymmetric cloud core towards the formation of a first core led to a $r^{-2}$ density distribution for the first core for $r>100$ AU (Furuya et al. 2012). Since we do not resolve the inner $r<700$ AU of Cha-MMS1, a simple, $r^{-2}$ density profile is probably adequate for describing the envelope of Cha-MMS1.

Such density profiles have also been observed in dense cores. Density distributions close to a $r^{-2}$ profile were derived for the starless cores L1498 and L1517B in Taurus (Tafalla et al. 2004). Bacmann et al. (2000) and Alves et al. (2001) also concluded that low-mass prestellar cores are described well by a density profile following an $r^{-2}$ dependence, but excluding the sharp edges and flattened centre. Sharp density edges have been observed at the edges of starless cores, with exponents as steep as $r^{-3.5}$ (Nielbock et al. 2012). For simplicity, we do not account for steep outer density slopes.

The mass of Cha-MMS1 was derived from the LABOCA $870 \mu \mathrm{m}$ continuum map of the Chamaeleon I cloud. The flux density measured within a radius of 3750 AU gives a mass of $\sim 1.44 M_{\odot}$ for the Cha-MMS1 core (Belloche et al. 2011a). We use this value to scale our input density profile.

\subsubsection{Inner turbulent broadening and isotopic ratios}

In Sect. 3.4, we found that the non-thermal dispersion for all the observed transitions shows no significant spatial variations for radii up to $\sim 12500 \mathrm{AU}(\sim 0.06 \mathrm{pc})$. We adopt a uniform turbulent broadening up to this radius and keep the shape of the outer profile for $12500 \mathrm{AU}<r<60000 \mathrm{AU}(\sim 0.3 \mathrm{pc})$ as a free parameter. The non-thermal dispersion appears to be uniform on scales $\sim 0.1 \mathrm{pc}$ within the interiors of dense cores 

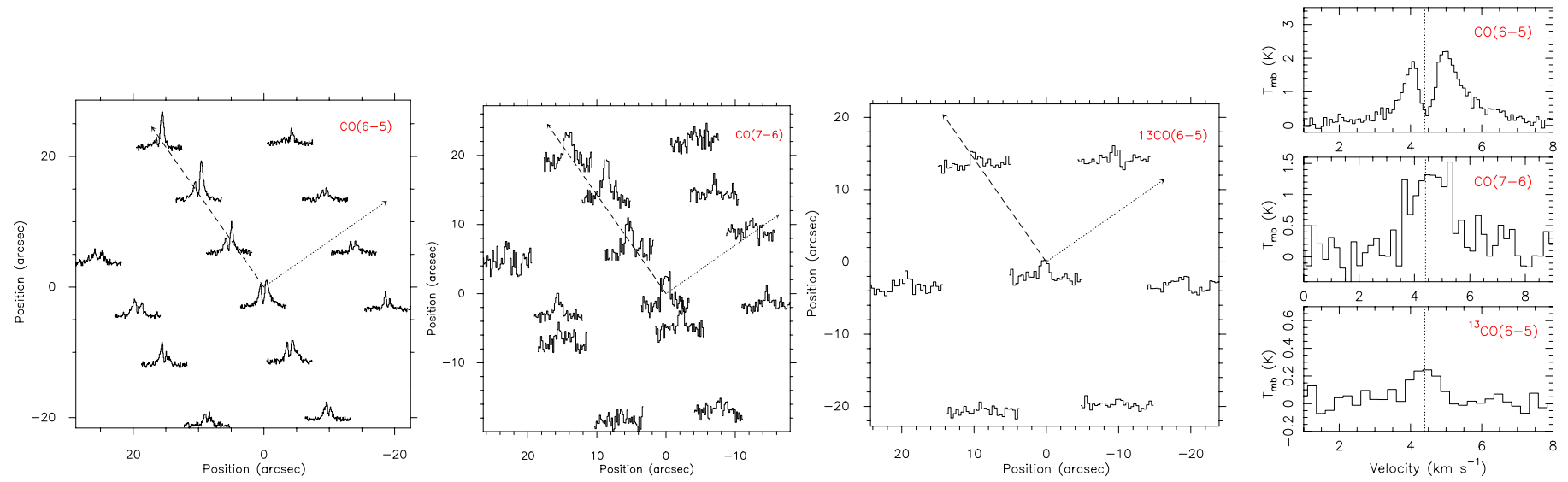

Fig. 9. CO 6-5, CO 7-6, and ${ }^{13} \mathrm{CO} 6-5$ spectra observed with the APEX telescope. The central position of Cha-MMS1 is at $(0,0)$. The directions parallel and perpendicular to the filament are shown as dashed and dotted arrows, respectively. The central spectra of the three transitions are plotted in the rightmost panel on the scale of the main-beam brightness temperature. The dotted line shows the systemic velocity of Cha-MMS1 derived from a seven-component hyperfine fit to the $\mathrm{N}_{2} \mathrm{H}^{+} 1-0$ multiplet and corrected for the $0.1 \mathrm{~km} \mathrm{~s}^{-1}$ velocity shift (see Sect. 3.2.1).

(e.g., Barranco \& Goodman 1998; Goodman et al. 1998) with increasing dispersion on larger scales (e.g., Goodman et al. 1998) that follows the Larson scaling law (Larson 1981).

We assume the local ISM abundance isotopic ratios: ${ }^{12} \mathrm{C} /{ }^{13} \mathrm{C} \sim 77$ (Wilson \& Rood 1994), ${ }^{32} \mathrm{~S} /{ }^{34} \mathrm{~S} \sim 22$ (Frerking et al. 1980), ${ }^{16} \mathrm{O} /{ }^{18} \mathrm{O} \sim 560$ (Wilson \& $\operatorname{Rood} 1994$ ), and ${ }^{18} \mathrm{O} /{ }^{17} \mathrm{O} \sim 4.11$ (Wouterloot et al. 2005).

\subsection{CS Modelling}

The following transitions of CS and its isotopologues were modelled: CS 2-1 (Mopra), $\mathrm{C}^{34} \mathrm{~S} 2-1$ (Mopra), and CS 5-4 (APEX). ${ }^{13} \mathrm{CS} 2-1$ and $\mathrm{C}^{33} \mathrm{~S} 2-1$ were only used as upper limits due to their non-detections and are not presented here. We perform the modelling of the spectra for the direction perpendicular to the filament, along which five positions were observed for CS 5-4 and seven for the other transitions. Figure 10 shows one of the "best fit" models (hereafter "MCS" model) for the CS molecular transitions. The distributions of density, abundance, kinetic temperature, radial velocity, and turbulent broadening characterising "MCS" can be seen in Fig. 11.

\subsubsection{Abundance}

The outer and inner abundances are constrained by the CS 2-1 absorption dip and the CS 5-4 intensity, respectively. In between, the abundance distribution is constrained by the $\mathrm{C}^{34} \mathrm{~S} 2-1$ spectra, which require a CS abundance of $2.5 \times 10^{-9}$ at a radius of $8000 \mathrm{AU}$. The depth of the CS 2-1 self-absorption is mostly determined by the outer abundance distribution of the low-excitation material at radii $\sim 10000 \mathrm{AU}<r<30000 \mathrm{AU}$. We do not constrain the CS abundance at radii larger than 30000 AU. CS 5-4 probes the innermost parts of the core and sets constraints on the abundance at radii $r<2000 \mathrm{AU}$. As a result, the CS abundance increases up to $8000 \mathrm{AU}$ and drops by a factor of 4 in the outer parts. As long as the temperature is low enough, CS and other sulphur-bearing molecules are expected to be strongly depleted towards the centre of dense cores where the density is high (e.g., Tafalla et al. 2004; Bergin et al. 2001). Depletion occurs due to the freezing-out process onto the dust grains (e.g., Tafalla et al. 2002; Stahler \& Yen 2010) and observations of starless cores, such as L1544, or Class 0 protostars, such as IRAM 04191, also showed CS depletion towards the centre by a factor of $\sim 20$ (Tafalla et al. 2002; Belloche et al. 2002).

\subsubsection{Turbulence}

We keep the turbulent broadening uniform at $0.3 \mathrm{~km} \mathrm{~s}^{-1}$ (FWHM) up to $\sim 12600 \mathrm{AU}$ as we see no clear variations in our diagrams of turbulent linewidth versus position up to this radius (see Fig. 6). We then let the turbulent linewidth double its value from $12600 \mathrm{AU}$ to the outermost envelope radius at $60000 \mathrm{AU}$. This is a constraint we derive from both the width of the CS 2-1 absorption dip and the CO 3-2 modelling (see Sect. 4.4).

\subsubsection{Opacities and excitation temperatures}

All transitions of CS and its isotopologue are optically thin on all positions apart from CS 2-1 (see Fig. 12). The model excitation temperatures are shown in Fig. 13. CS $2-1, C^{34} \mathrm{~S} 2-1$, and CS 5-4 are thermalised up to $\sim 2500 \mathrm{AU}, \sim 1500 \mathrm{AU}$, and $\sim 400$ AU, respectively.

\subsubsection{Discrepancies between model and observations}

The model reproduces the absorption dip of CS 2-1 well for all positions, but, the intensity of the blue peak at the central position and at $-17.5^{\prime \prime}$ is higher compared to the observations. The discrepancy is not that strong at $+17.5^{\prime \prime}$, and the model fits the peak temperature well at the outer positions, i.e., $\pm 35^{\prime \prime}$ and $\pm 52.5^{\prime \prime}$. A peculiarity of the observed CS $2-1$ spectra is the existence of velocity wings at all positions. The velocity wings are not reproduced by the model and may partly arise from extended emission stemming from the outflow of the nearby Class I object (see Fig. 1). It is, however, unclear whether this can explain both the redshifted and blueshifted wing emissions. The $\mathrm{C}^{34} \mathrm{~S} 2-1$ model is weaker at the outermost $\pm 52.5^{\prime \prime}$ positions while matching the intensity of the spectra at the other positions well. CS 5-4, on the other hand, shows one more peculiar feature: the model does not account for the excess of redshifted emission that is prominent toward the central position. 


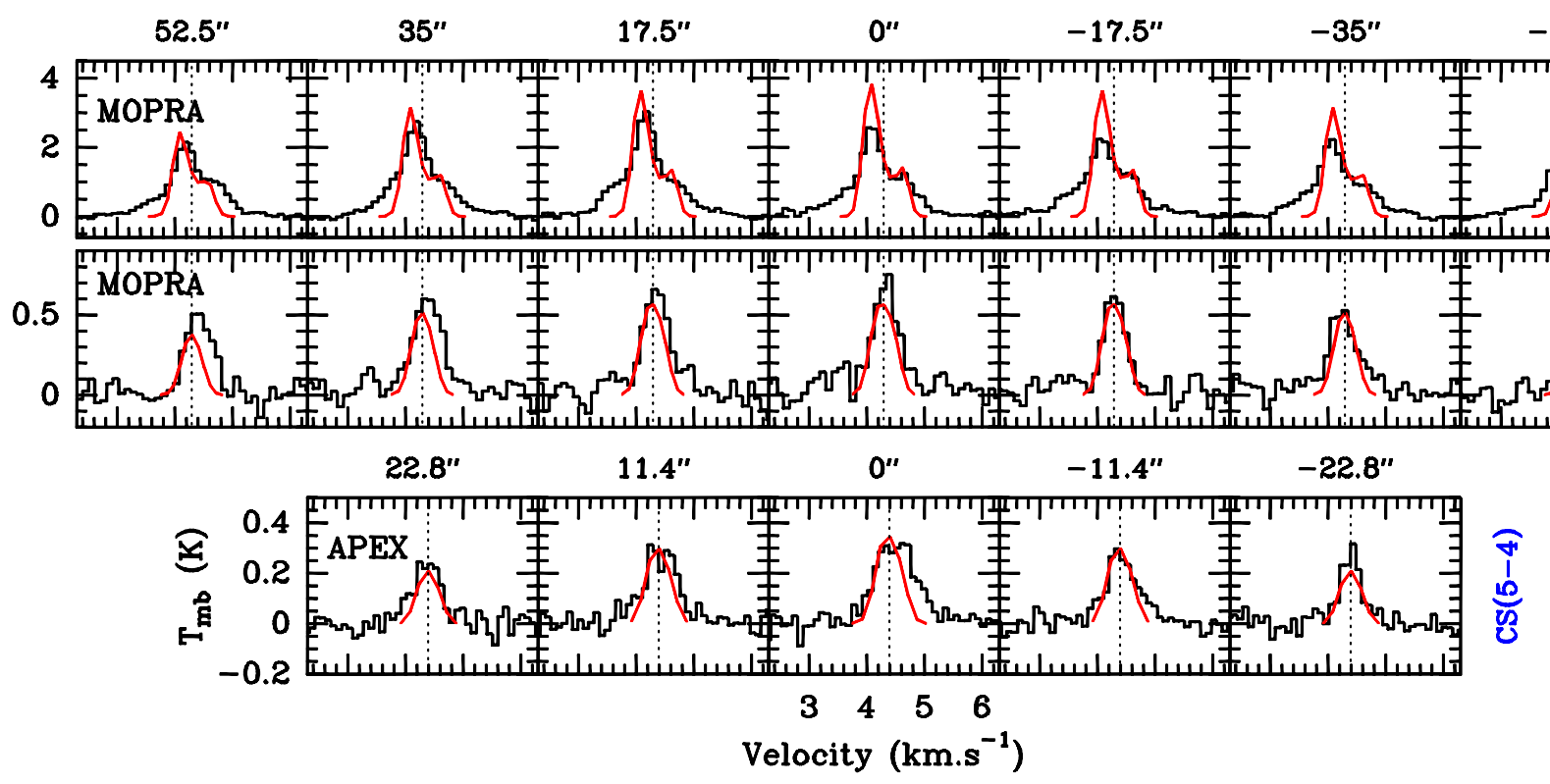

Fig. 10. Best fit model "MCS" for the CS and $C^{34} S$ molecular transitions. The black lines represent the observed spectra, while the red spectra are generated by the MAPYSO radiative transfer code. The dotted line shows the systemic velocity of Cha-MMS1 derived from a seven-component hyperfine fit to the $\mathrm{N}_{2} \mathrm{H}^{+} 1-0$ multiplet. For the APEX spectra, a correction of $0.1 \mathrm{~km} \mathrm{~s}^{-1}$ was added (see Sect. 3.2.1). The angular separation of the different positions with respect to the central spectrum is shown on top of the respective spectra. The model assumes spherical symmetry, so the model spectra at symmetric positions are identical. The telescope used for conducting the obervations is shown at the leftmost box of each row. The spectra from left to right correspond to the south-east to north-west direction perpendicular to the filament.
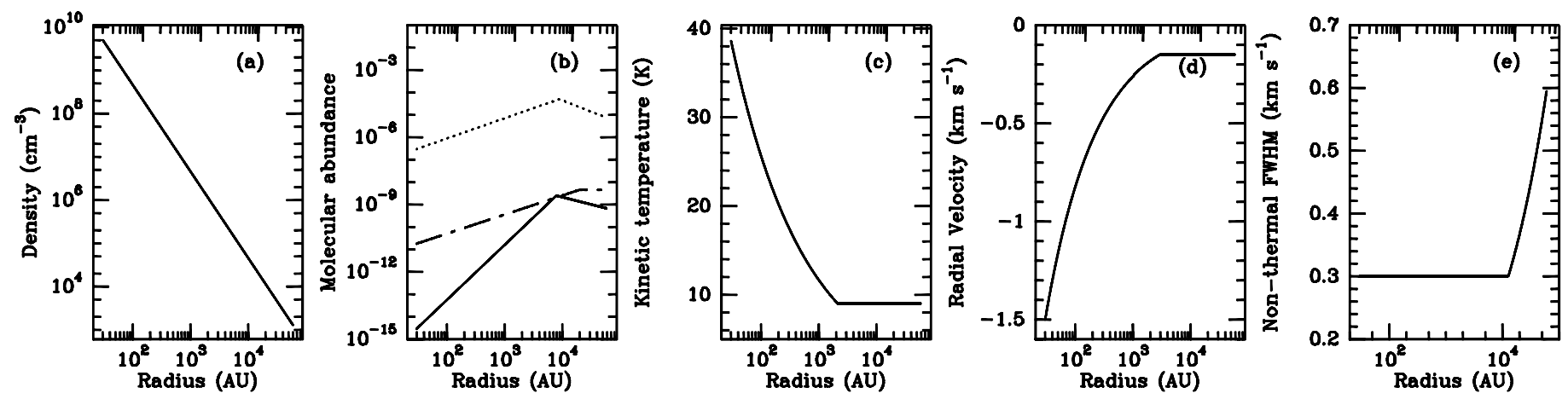

Fig. 11. Input profiles for the best-fit models "MCS", "MHCOP", and "MCO": a) density, b) CS (solid), HCO' (dash-dot), and CO (dot) abundance; c) kinetic temperature; d) radial velocity; and e) turbulent linewidth.

\subsubsection{Testing the infall velocity field}

Despite the discrepancies, the "MCS" model provides a consistent overall fit to the data. As we are interested in the infall velocity structure of the core we input different infall velocity profiles to "MCS" in order to test and constrain its value. The linewidths of the low optical depth lines and the position of the absorption dip of the optically thick lines can be used to place relatively tight constraints on the velocity structure of the source. We first test different uniform infall velocity profiles and then velocity profiles with a free-fall, power-law $\left(v \propto r^{-0.5}\right)$ dependence in the inner parts.

In the case of uniform velocities, the best agreement is found for velocities in the range $0.1 \mathrm{~km} \mathrm{~s}^{-1}-0.2 \mathrm{~km} \mathrm{~s}^{-1}$. For velocities less than $0.1 \mathrm{~km} \mathrm{~s}^{-1}$ the peak asymmetry of the CS $2-1$ spectra reduces considerably with the red peak becoming too strong in comparison to the observations. In addition, the linewidths of both the CS $2-1$ and $\mathrm{C}^{34} \mathrm{~S} 2-1$ lines become narrower than the observed linewidths. In contrast, when the infall velocities exceed $0.2 \mathrm{~km} \mathrm{~s}^{-1}$, the blue peak of CS 2-1 becomes much broader and the central $\mathrm{C}^{34} \mathrm{~S} 2-1$ spectrum starts showing a double-peaked structure (also seen in the opacity profile), which is inconsistent with the observations.

We then apply a power law with an exponent of -0.5 for the inner parts at gradually increasing radii while keeping the velocity constant at the outer parts in order to test whether higher inner velocities are consistent with the observations. From now on, we refer to the radius at which the velocity profile changes from a power-law to a uniform $r$ dependence as the "breakpoint". Our tests suggest that such a power law velocity distribution matches the observations for radii up to $9000 \mathrm{AU}$ for velocities in the range of $v_{\text {break }}=0.1 \mathrm{~km} \mathrm{~s}^{-1}-0.2 \mathrm{~km} \mathrm{~s}^{-1}$, after which we let the velocity remain uniform. For breakpoint radii exceeding $9000 \mathrm{AU}$, the wide spatial range of high infall velocities produces broader CS 2-1 linewidths and stronger red peak intensities than the observed spectra. The CS 5-4 and $\mathrm{C}^{34} \mathrm{~S} 2-1$ models also start showing a double-peaked structure, which disagrees with the observations.

Therefore, the CS modelling suggests that the core's envelope is infalling with subsonic to transonic outer velocities of $0.1 \mathrm{~km} \mathrm{~s}^{-1}-0.2 \mathrm{~km} \mathrm{~s}^{-1}$. Inner free-fall power-law velocity 
distributions are possible with breakpoints at $r \leq 9000 \mathrm{AU}$, with infall velocities reaching supersonic values at $r \leq 3500 \mathrm{AU}$ in this case. We do not constrain the infall velocity structure of the envelope for radii greater than $30000 \mathrm{AU}$. The infalling motions at the outer parts of the envelope $(<30000 \mathrm{AU})$ contradict the Shu (1977) assumption of a static envelope in the inside-out collapse model.

The radial velocity structure of the "MCS" model shown in Fig. 11 corresponds to an $r^{-0.5}$ dependence for radii $<3000 \mathrm{AU}$, and a uniform infall velocity of $0.15 \mathrm{~km} \mathrm{~s}^{-1}$ for $3000 \mathrm{AU} \leq r \leq$ 60000 AU (see Fig. 11d).

\section{3. $\mathrm{HCO}^{+}$Modelling}

The following molecular transitions of $\mathrm{HCO}^{+}$and its isotopologues were modelled: $\mathrm{HCO}^{+} 1-0, \mathrm{HCO}^{+} 3-2, \mathrm{H}^{13} \mathrm{CO}^{+} 1-0$, $\mathrm{H}^{13} \mathrm{CO}^{+} 3-2, \mathrm{H}^{13} \mathrm{CO}^{+} 4-3$, and $\mathrm{HC}^{18} \mathrm{O}^{+} 1-0$. The $1-0$ transitions were observed with Mopra, the other ones with APEX. We have central position spectra for $\mathrm{HCO}^{+}, \mathrm{H}^{13} \mathrm{CO}^{+}$, and $\mathrm{HC}^{18} \mathrm{O}^{+} 1-0$, while $\mathrm{HCO}^{+}$and $\mathrm{H}^{13} \mathrm{CO}^{+} 3-2$ were also observed at seven and $\mathrm{H}^{13} \mathrm{CO}^{+} 4-3$ at five positions along the direction perpendicular to the filament (see Fig. 1). One of the models that provides a good fit to the data is shown in Fig. 14, and hereafter we refer to it as "MHCOP". The distributions of density, abundance, kinetic temperature, radial velocity, and turbulent broadening characterising "MHCOP" can be seen in Fig. 11.

\subsubsection{Abundance}

We take the following observational constraints into account to define the input abundance profile (Fig. 11b). The inner profile is constrained by the intensities of the optically thin $\mathrm{H}^{13} \mathrm{CO}^{+} 3-2$, $\mathrm{H}^{13} \mathrm{CO}^{+} 4-3$, and $\mathrm{HC}^{18} \mathrm{O}^{+} 1-0$ spectra.

A decrease in the $\mathrm{HCO}^{+}$abundance by a factor of $\sim 20$ from a radius of $20000 \mathrm{AU}$ down to the centre fits the intensities of the optically thin lines well. As a test, we extended the plateau of uniform outer abundance towards the centre to radii $r<20000 \mathrm{AU}$, and found that this produced model intensities that were too high for the optically thin spectra. By varying the abundance profile between $20000 \mathrm{AU}$ and $60000 \mathrm{AU}$, we conclude that we do not constrain the outer, $r \geq 20000 \mathrm{AU}$, abundance profile with our current $\mathrm{HCO}^{+}$transitions. We therefore use a uniform outer abundance for $r \geq 20000 \mathrm{AU}$ as an approximation.

\subsubsection{Opacities and excitation temperatures}

Figures 15 and 16 show the resulting opacity and excitation temperature profiles of the model for each transition. $\mathrm{HCO}^{+} 3-2, \mathrm{HCO}^{+} 1-0$, and $\mathrm{H}^{13} \mathrm{CO}^{+} 1-0$ are optically thick, while $\mathrm{H}^{13} \mathrm{CO}^{+} 4-3, \mathrm{H}^{13} \mathrm{CO}^{+} 3-2$, and $\mathrm{HC}^{18} \mathrm{O}^{+} 1-0$ are in the optically thin range. $\mathrm{HCO}^{+} 1-0$ is thermalised up to $\sim 10000 \mathrm{AU}$ and $\mathrm{HCO}^{+} 3-2, \mathrm{H}^{13} \mathrm{CO}^{+} 1-0$, and $\mathrm{HC}^{18} \mathrm{O}^{+} 1-0$ are thermalised up to $\sim 3000 \mathrm{AU}$. $\mathrm{H}^{13} \mathrm{CO}^{+} 4-3$ and $\mathrm{H}^{13} \mathrm{CO}^{+} 3-2$ cease to be thermalised for radii larger than $\sim 500 \mathrm{AU}$. $\mathrm{HCO}^{+} 1-0$ is thermalised up to a much larger radius than $\mathrm{H}^{13} \mathrm{CO}^{+}$and $\mathrm{HC}^{18} \mathrm{O}^{+} 1-0$ because of its much higher optical depth.

\subsubsection{Discrepancies between model and observations}

One of the major discrepancies between model and observations is the failure of the model to reproduce the emission of the red peak of the $\mathrm{HCO}^{+} 3-2$ transition. Raising the outer temperature of the core does not have a significant effect on the red peak of the model spectrum since this transition is not thermally excited at radii larger than $3000 \mathrm{AU}$. This disagreement might then be a density effect instead, if the true density profile deviates from the spherically symmetric, $r^{-2}$ dependence. The observed $\mathrm{H}^{13} \mathrm{CO}^{+} 3-2$ spectra show higher intensities in the north-west $\left(-11.4^{\prime \prime},-22.8^{\prime \prime},-34.2^{\prime \prime}\right)$ direction than in the south-east direction. $\mathrm{H}^{13} \mathrm{CO}^{+} 3-2$ is not the only transition that does not peak at the central position. Higher intensities toward the north-west are also seen in our $\mathrm{N}_{2} \mathrm{H}^{+} 1-0$ spectra. The $\mathrm{N}_{2} \mathrm{H}^{+} 1-0$ emission in the maps presented by Ladd et al. (2011) is similarly not peaked at Cha-MMS1. "MHCOP" fits the spectra on the north-west side well, but it does not fit the excess emission on the south-east side. We cannot yet pinpoint the reason for this intensity difference on each side of the central spectrum. $\mathrm{H}^{13} \mathrm{CO}^{+} 4-3$ and the central spectrum of $\mathrm{HC}^{18} \mathrm{O}^{+} 1-0$ are reproduced rather well, as is $\mathrm{H}^{13} \mathrm{CO}^{+} 1-0$, even though the exact lineshape of the observed spectrum is not reproduced. The observed $\mathrm{H}^{13} \mathrm{CO}^{+} 1-0$ is broader than the model at the redshifted part of the spectrum, like CS 5-4 (see Sect. 4.2).

The model strongly overestimates the peak temperature of the observed $\mathrm{HCO}^{+} 1-0$ transition which is even weaker than $\mathrm{H}^{13} \mathrm{CO}^{+} 1-0 . \mathrm{HCO}^{+} 1-0$ is very optically thick and thermalised up to large radii. It is certainly affected by significant absorption from the low-density material in the ambient cloud at $r \geq$ $60000 \mathrm{AU}$, which is not included in the modelling.

\subsubsection{Testing the infall velocity field}

There is a range of infall velocity profiles that give consistent fits to the data. In the case of uniform, constant velocities up to $60000 \mathrm{AU}$ the range $0.1 \mathrm{~km} \mathrm{~s}^{-1}-0.2 \mathrm{~km} \mathrm{~s}^{-1}$ agrees well with the observations. For values higher than $0.2 \mathrm{~km} \mathrm{~s}^{-1}$ the $\mathrm{HCO}^{+} 3-2, \mathrm{H}^{13} \mathrm{CO}^{+} 3-2, \mathrm{H}^{13} \mathrm{CO}^{+} 4-3$, and $\mathrm{HC}^{18} \mathrm{O}^{+} 1-0$ spectra become too broad while for velocities lower than $0.1 \mathrm{~km} \mathrm{~s}^{-1}$ the absorption dip is not redshifted enough. We then tested a velocity profile described by a power law following an $r^{-0.5}$ dependence in the inner part of the core up to a certain radius, after which the infall velocity remains uniform, as we did for CS. For a breakpoint at 3000 AU infall velocities of $v_{\text {break }}=0.1 \mathrm{~km} \mathrm{~s}^{-1}-0.2 \mathrm{~km} \mathrm{~s}^{-1}$ give consistent fits. When we increase the breakpoint radius, the range of consistent infall velocities decreases. At $6000 \mathrm{AU}$ the breakpoint velocities still consistent with the data are $0.1 \mathrm{~km} \mathrm{~s}^{-1}-0.15 \mathrm{~km} \mathrm{~s}^{-1}$ and at $9000 \mathrm{AU}, 0.1 \mathrm{~km} \mathrm{~s}^{-1}$. Overall, inner power-law profiles at radii larger than $9000 \mathrm{AU}$ produce spectra with too broad linewidths for all transitions, apart from $\mathrm{H}^{13} \mathrm{CO}^{+} 1-0$, and inconsistent double-peaked spectra for $\mathrm{H}^{13} \mathrm{CO}^{+} 3-2, \mathrm{H}^{13} \mathrm{CO}^{+} 4-3$, and $\mathrm{HC}^{18} \mathrm{O}^{+} 1-0$. We also varied the outer infall velocity profile to check whether it is constrained by our data. To accomplish this we let the infall velocity drop sharply to zero at gradually increasing radii and found that the model does not constrain the velocities for radii greater than $35000 \mathrm{AU}$. Even though the dip in the $\mathrm{HCO}^{+} 3-2$ model spectrum in Fig. 14 is not as redshifted as in the observed spectrum, a velocity drop to zero at radii smaller than $35000 \mathrm{AU}$ makes this discrepancy even stronger by producing model spectra with almost no apparent redshift of the $\mathrm{HCO}^{+} 3-2$ absorption dip. We therefore cannot accurately constrain the infall velocity structure of the core for $r \geq 35000 \mathrm{AU}$ from the $\mathrm{HCO}^{+}$modelling.

The velocity distribution corresponding to model "MHCOP" is the same as the one of "MCS" (see Fig. 11d). 


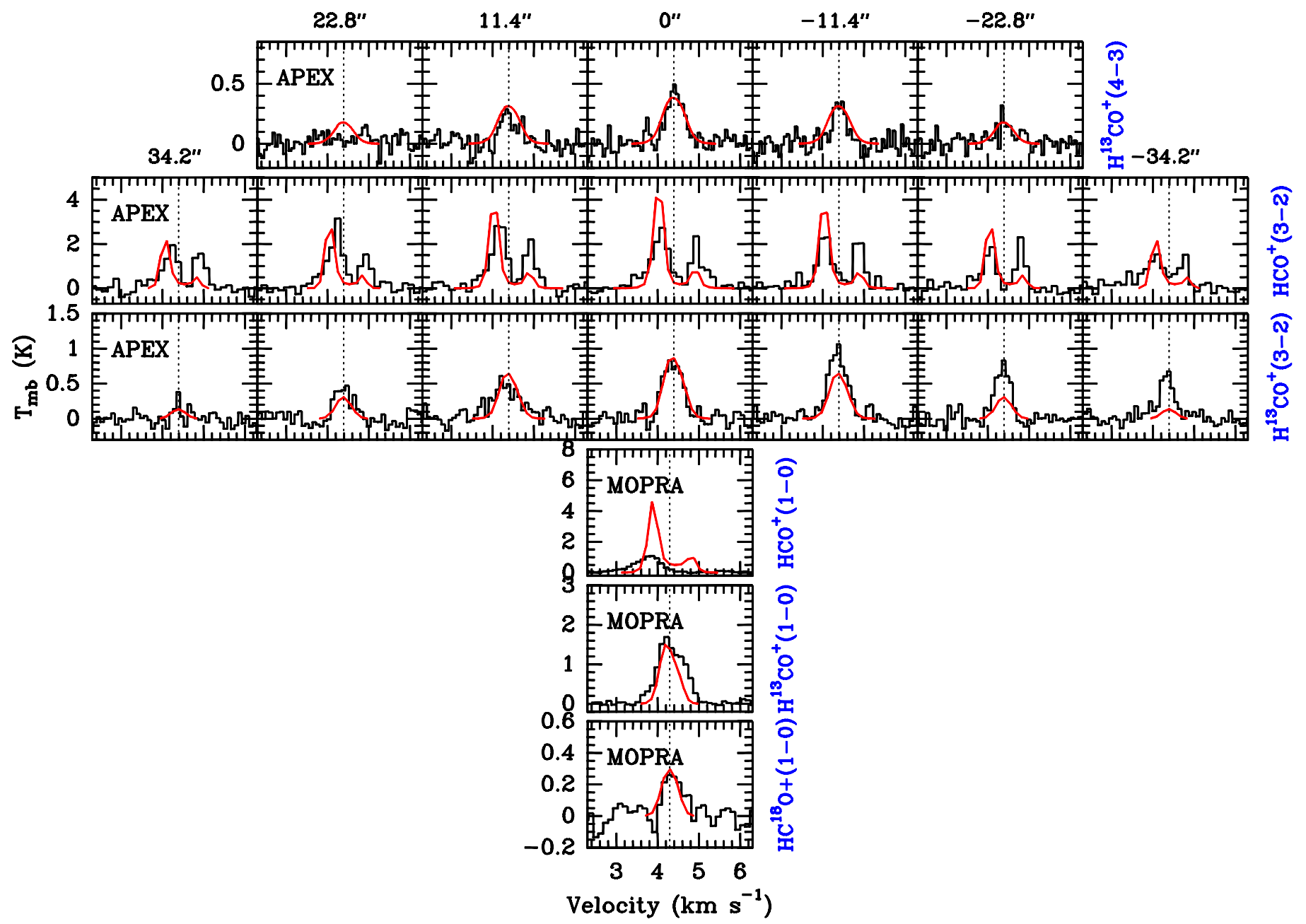

Fig. 14. Same as Fig. 10 but for the best fit model "MHCOP".

\subsection{CO Modelling}

We modelled the following molecular transitions of $\mathrm{CO}$ and its isotopologues: $\mathrm{CO} 3-2, \mathrm{CO} 4-3, \mathrm{CO} 6-5, \mathrm{CO} 7-6,{ }^{13} \mathrm{CO} 6-5$, and $\mathrm{C}^{18} \mathrm{O} 2-1$. Figure 17 shows one of the models that fits the data relatively well, hereafter "MCO". The distributions of density, abundance, kinetic temperature, radial velocity, and turbulent broadening characterising "MCO" can be seen in Fig. 11.

\subsubsection{Abundance}

The abundance profile of $\mathrm{CO}$ down to a radius of $\sim 1000 \mathrm{AU}$ is mainly constrained by the intensity variations of the $\mathrm{C}^{18} \mathrm{O}$ and $\mathrm{C}^{17} \mathrm{O} 2-1$ transitions. The ratio of the $\mathrm{C}^{17} \mathrm{O}$ and $\mathrm{C}^{18} \mathrm{O} 2-1$ integrated intensities yields $\mathrm{C}^{18} \mathrm{O} 2-1$ opacities ranging from $\sim 1.6$ toward the centre to less than $\sim 0.5$ toward the outer parts (see Appendix B.1). We take these opacity values into account to further constrain our model. Finally, the optically thin ${ }^{13} \mathrm{CO} 6-5$ transition, which traces higher densities and is spatially better resolved, sets strong constraints on the abundance in the inner parts of the envelope ( $r<1500 \mathrm{AU})$. Overall, we obtain the abundance profile shown in Fig. 11b, with a maximum of $5 \times 10^{-5}$ at $9000 \mathrm{AU}$, depletion by a factor of $\sim 10$ from $\sim 9000 \mathrm{AU}$ to $\sim 700 \mathrm{AU}$, and also a decrease in the abundance by a factor $\sim 7$ from $\sim 9000$ AU to $\sim 20000 \mathrm{AU}$, in order to match the lower outer $\mathrm{C}^{18} \mathrm{O}$ opacity. We constrain the $\mathrm{CO}$ abundance up to $20000 \mathrm{AU}$. CO depletion has previously been observed towards the inner parts of various prestellar cores (e.g., Bacmann et al. 2002; Christie et al. 2012).

Furuya et al. (2012) coupled a gas-grain chemical network to $3 \mathrm{D}$ radiation hydrodynamic simulations to derive molecular abundances at the first core stage. In their model, CO desorbs to the gas phase at a temperature of $25 \mathrm{~K}$, somewhat higher than the commonly used $20 \mathrm{~K}$ value, due to the high density of the first-core envelope. The density at which their simulation reaches $25 \mathrm{~K}$ is $\sim 10^{9} \mathrm{~cm}^{-3}$, which is similar in our model. We tested the effect of an inner $\mathrm{CO}$ desorption region at $r \leq 54 \mathrm{AU}$ (from Eq. (7)), at which radius the temperature reaches $25 \mathrm{~K}$ in our model. We used an inner $\mathrm{CO}$ abundance of $1 \times 10^{-4}$ for the desorption region, which is consistent with the predicted abundance for the first core stage (Furuya et al. 2012). At radii larger than $54 \mathrm{AU}$, and therefore at temperatures $\mathrm{T}<25 \mathrm{~K}, \mathrm{CO}$ depletion takes place. We found that the model is not sensitive to the abundance within the inner $54 \mathrm{AU}$ and the presence or the absence of a desorption region on these smaller scales has no apparent effect on the spectra. Therefore, we do not account for inner desorption.

\subsubsection{Turbulence}

The self-absorption of the CO 3-2 spectra is very broad, suggesting a large turbulent broadening in the outer parts (radii $r \geq 12500 \mathrm{AU})$. We therefore raise it by a factor of $\sim 2$ from $12500 \mathrm{AU}$ to $60000 \mathrm{AU}$. In this way, the blueshifted side of 
A. E. Tsitali et al.: The dynamical state of the first hydrostatic core candidate Chamaeleon-MMS1

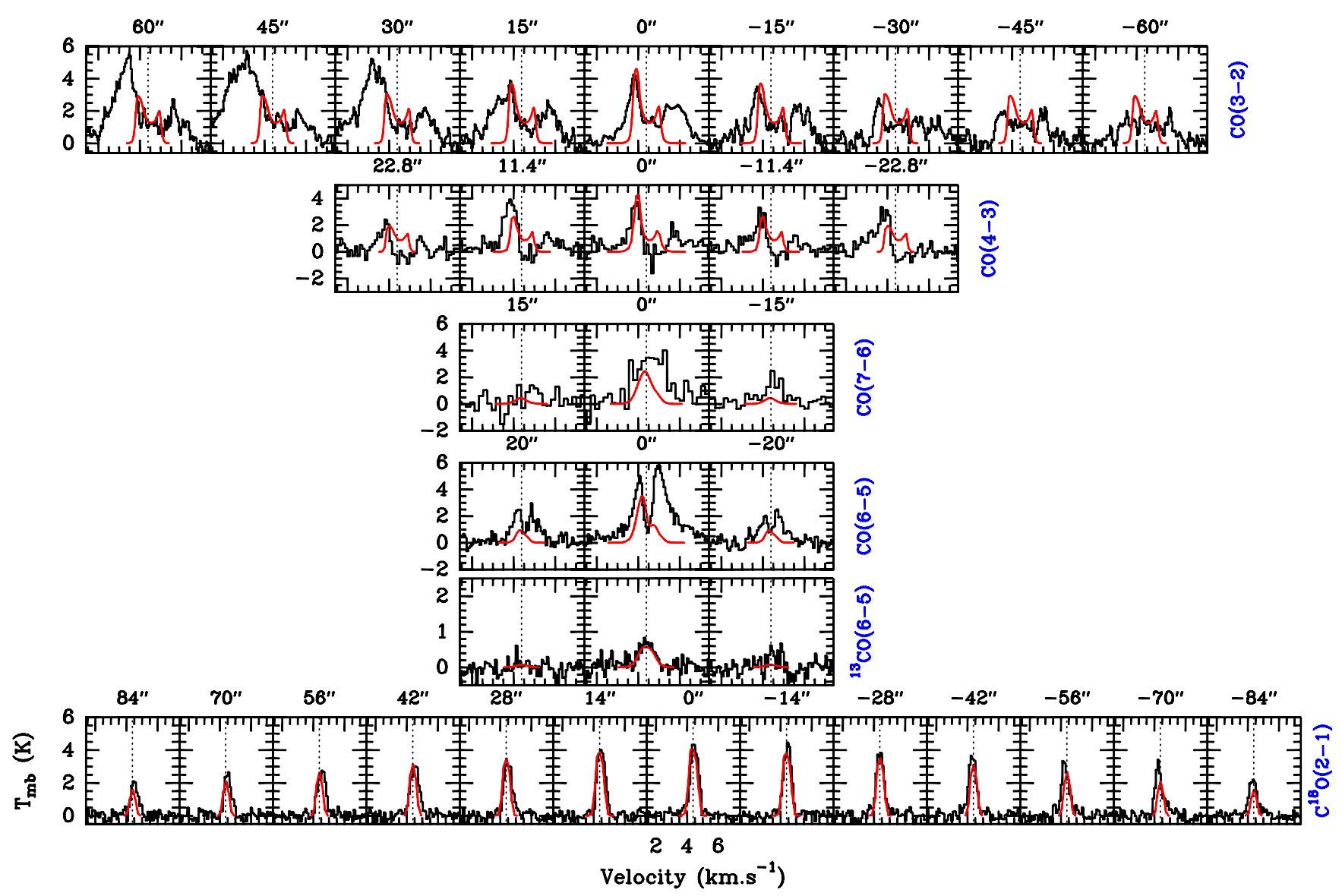

Fig. 17. Same as Fig. 10 but for the best fit model "MCO" of the CO transitions.

the self-absorption is reproduced well, but the redshifted side of the model is still too narrow. The turbulent broadening is kept uniform for radii $r \leq 12500$ AU (see Fig. 11), as derived in Sect. 3.4.

\subsubsection{Model opacities and excitation temperatures}

Figures 18 and 19 show the model opacities and excitation temperatures for all the transitions. $\mathrm{CO} 3-2$, CO 4-3, and $\mathrm{C}^{18} \mathrm{O} 2-1$ remain thermalised out to $\sim 20000 \mathrm{AU}, \sim 13000 \mathrm{AU}$, and $\sim 9000 \mathrm{AU}$, respectively, while $\mathrm{CO} 6-5$ and $\mathrm{CO} 7-6$ cease to be thermalised at $\sim 2000 \mathrm{AU}$. Moreover, all the transitions are optically thick with the exception of ${ }^{13} \mathrm{CO} 6-5$ at all positions, CO 7-6 at offset positions, and $\mathrm{C}^{18} \mathrm{O} 2-1$ at the outermost position (see Fig. 18).

\subsubsection{Discrepancies between model and observations}

The spectra of CO 3-2 at 15", 30", 45", and 60" show strong emission in the blueshifted part of the spectrum, while the spectra at $-15^{\prime \prime},-30^{\prime \prime},-45^{\prime \prime}$, and $-60^{\prime \prime}$ show much weaker blueshifted emission. Excess emission in the redshifted part of the spectra is also seen at all positions. We expect the outflow of the neighbouring Class I protostar (see Fig. 1) to contaminate the low-density tracers such as CO 3-2, especially at the offset positions, thereby broadening the observed emission. Belloche et al. (2006) presented a CO 3-2 intensity map of the region toward Cha-MMS1 (see Fig. 1 of their paper). The blueshifted emission they show reaches our offset positions, and especially affects the spectra towards the south-east of Cha-MMS1. They also discuss the possible presence of two separate outflows in this region. The highly broadened blue and red peaks of CO 3-2 might partly result due to these outflows if they have a wide opening angle and lie close to the plane of the sky (see Cabrit \& Bertout 1990).

The model reproduces the $\mathrm{C}^{18} \mathrm{O} 2-1$ emission well, and its opacity, as well as the ${ }^{13} \mathrm{CO} 6-5$ emission. However, the model fails to reproduce the strong redshifted emission of the $\mathrm{CO}$ 6-5 and 7-6 transitions, similar to the CS 5-4 emission. Because these transitions probe regions of high densities, this extra emission may be an indication of additional warmer, high-velocity material in the inner core (see Fig. 10). This emission may stem from a very compact outflow. To address this question, however, we would need to resolve the very inner part of the core on small scales of a few hundred AU (see Sect. 1.1).

\subsubsection{Testing the infall velocity field}

From the $\mathrm{CO}$ modelling we draw the following conclusions on the infall velocity structure across the envelope. We find that uniform infall velocities of up to $0.2 \mathrm{~km} \mathrm{~s}^{-1}$ give fits relatively consistent with the data. Velocities above $0.2 \mathrm{~km} \mathrm{~s}^{-1}$ produce $\mathrm{C}^{18} \mathrm{O} 2-1$ linewidths that are too broad compared to observed values. We test an inner power-law velocity dependence $(v \propto$ $r^{-0.5}$ ), with uniform outer velocities. We find that the power-law profile is consistent with the observations up to $\sim 6000 \mathrm{AU}$ if the infall velocity is in the range of $v_{\text {break }}=0.1 \mathrm{~km} \mathrm{~s}^{-1}-0.15 \mathrm{~km} \mathrm{~s}^{-1}$ at this radius, after which it remains constant. A radius of $9000 \mathrm{AU}$ is also consistent when $v_{\text {break }}=0.1 \mathrm{~km} \mathrm{~s}^{-1}$ at this breakpoint and onwards. For larger radii the model spectra are much broader than the observed spectra due to the extended 


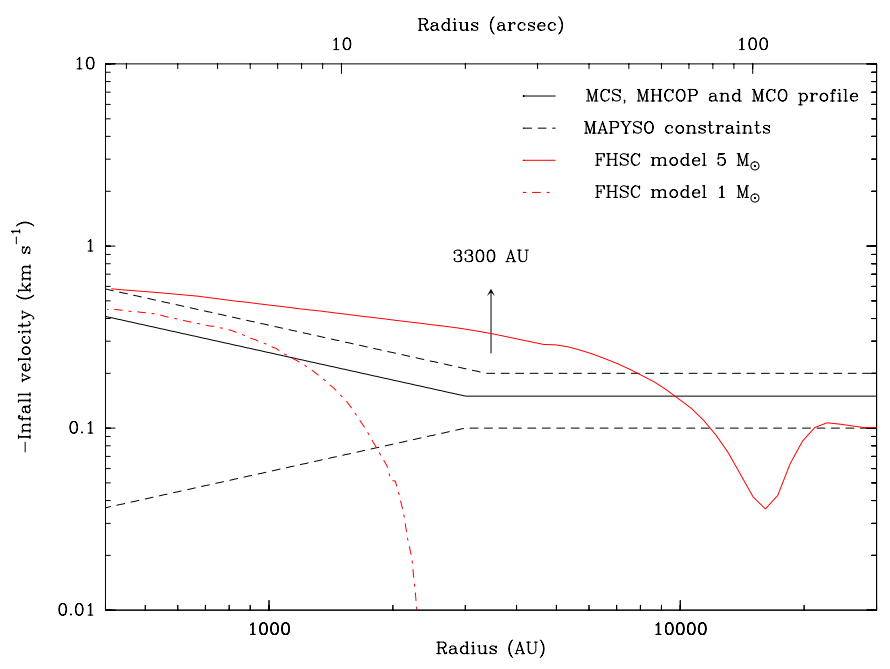

Fig. 20. Infall velocity field of the envelope of Cha-MMS1 based on the radiative transfer modelling with MAPYSO. The solid black line corresponds to the velocity profile of the "MCS", "MHCOP", and "MCO" models. The dashed lines show the range of velocity fields that are still consistent with the observations. The red solid and dashed curves correspond to the average radial velocity at the equatorial plane of a magnetised FHSC with core masses of $1 M_{\odot}$ and $5 M_{\odot}$, and ages of $850 \mathrm{yr}$ and $2684 \mathrm{yr}$, respectively. (Commerçon et al. 2012, see details in Sect. 5.4.2).

spatial range of high inner infall velocities. However, the opacity of the optically thick transition CO 3-2 does not allow us to set constraints on the lower limit of the infall velocity.

The velocity profile that coresponds to the "MCO" model is shown in Fig. 11, and it is identical to the ones used for "MHCOP" and "MCS".

\subsection{Infall velocity distribution of Cha-MMS1: combining modelling results}

We obtain slightly different constraints on the infall velocity structure of Cha-MMS1 from the radiative transfer modelling of the $\mathrm{CS}, \mathrm{HCO}^{+}$, and $\mathrm{CO}$ molecular transitions. We take the envelope of the infall velocity profiles that are consistent with all three datasets as the overall range of possible infall velocities for Cha-MMS1. The consistent velocities are shown as the area enclosed in Fig. 20.

The infall velocity is relatively well constrained over the range of radii $3300 \mathrm{AU}$ to $30000 \mathrm{AU}$, with subsonic/transonic velocities in the range $0.1 \mathrm{~km} \mathrm{~s}^{-1}$ to $0.2 \mathrm{~km} \mathrm{~s}^{-1}$. Our data do not constrain the velocity field beyond $~ 30000$ AU. Below $3300 \mathrm{AU}$, there are more degeneracies, and the spectra are consistent with an increase in the infall velocity as $r^{-0.5}$, but also with a flat velocity profile, or even a decrease below a radius of $\sim 3000 \mathrm{AU}$.

\section{Discussion}

\subsection{Far-infrared emission and luminosity}

Recent radiation-MHD simulations predict that strongly or weakly magnetised FHSCs are expected not only to emit at $70 \mu \mathrm{m}$ but also to produce detectable emission at wavelengths down to $20 \mu \mathrm{m}$, especially during the latest phase of their evolution (Commerçon et al. 2012). Previous simulations that found no significant first-core emission below $30 \mu \mathrm{m}-50 \mu \mathrm{m}$ were either spherical (e.g., Masunaga et al. 1998; Omukai 2007) or did not take the magnetic field into account and used a barotropic equation of state (Saigo \& Tomisaka 2011). A first core can therefore also be identified by a Spitzer $24 \mu \mathrm{m}$ (and $70 \mu \mathrm{m}$ ) detection at late phases if its inclination is less than $60^{\circ}$ and there is no detection at wavelengths smaller than $20 \mu \mathrm{m}$ (Commerçon et al. 2012). As seen in Sect. 3.1, the $24 \mu \mathrm{m}$ and $70 \mu \mathrm{m}$ Spitzer fluxes of Cha-MMS1 are consistent with the predictions of the RMHD simulation of Commerçon et al. (2012) for a magnetised FHSC with a normalised mass-to-magneticflux ratio of 2 seen at an inclination lower than $60^{\circ}$. The SED of Cha-MMS1 is therefore consistent with Cha-MMS1 being at the FHSC stage. However, if its actual inclination is higher than $60^{\circ}$ then Cha-MMS1 would have to be in a more advanced stage (Class 0).

\subsection{Outflows}

Class 0 protostars usually drive fast, extended, and easily detectable outflows, whereas FHSCs are predicted to drive very compact, slow outflows (see Sect. 1.1). This is a major observational signature that can be used to distinguish between the two and break the degeneracies that remain in their SEDs when their inclination is not known.

A search for a fast, large-scale outflow driven by Cha-MMS1 in CO 3-2 with APEX was negative (Belloche et al. 2006). We observed the $\mathrm{CO} 6-5, \mathrm{CO} 7-6$, and ${ }^{13} \mathrm{CO} 6-5$ transitions in order to search for signs of a compact, unresolved outflow. The modelling of these transitions gave ambiguous, but maybe promising results (see Sect. 4.4). Our model does not reproduce the redshifted part of the $\mathrm{CO} 6-5$ and $7-6$ emission, while it fits the $\mathrm{C}^{18} \mathrm{O} 2-1$ transition well. This excess emission might point to the presence of unresolved, higher-velocity material at the inner core (see Shinnaga et al. 2009, for a similar case of excess emission in CO 6-5 and 7-6 toward the more evolved protostar L1521F). In addition to this, the CS 5-4 model in Sect. 4.2 shows a similar discrepancy. Its observed spectrum has an excess of redshifted emission that the model fails to reproduce, while it fits the lower- $J$ CS and $\mathrm{C}^{34} \mathrm{~S}$ transitions well. CS 5-4 probes material at higher densities, so that its broad spectrum indicates higher velocity, dense material close to the centre of the core, which cannot be seen with the other, lower density transitions.

Despite hints of the presence of dense, higher velocity material confined to the centre of the core, there is also an alternative explanation. Figure 21 compares the CO 6-5 spectra at the centre and in the north-east direction of the filament (hence toward the nearby Class I outflow) to the central spectrum of CS 5-4. The CO 6-5 red peak emission becomes stronger as we move up along the filament. It peaks at a velocity where CS 5-4 has some wing emission. This suggests that at least part of the excess emission in the CS 5-4 spectrum is not confined to the inner parts of Cha-MMS1 but instead extends towards the nearby Class I outflow lobe. The bulk of the CS 5-4 excess emission, however, peaks at a lower velocity, and it is unclear whether it represents a similar extended component. Higher-angular resolution observations are certainly needed to make any reliable conclusions about the presence of a compact, slow outflow driven by Cha-MMS1.

As mentioned in Sect. 4.2.4, all CS 2-1 spectra in Fig. 10 show blueshifted and redshifted wing emissions that are not accounted for by our model. Such wing emissions are even more pronounced in $\mathrm{CO} 3-2$ (Fig. 17), the blueshifted one being clearly associated with emission from the outflow driven by the nearby Class I protostar (Belloche et al. 2006). Hiramatsu et al. (2007) and Ladd et al. (2011) propose that the curved shape of the blue lobe of this outflow is due to its partial interaction with the Cha-MMS1 dense core. This would explain the presence of 


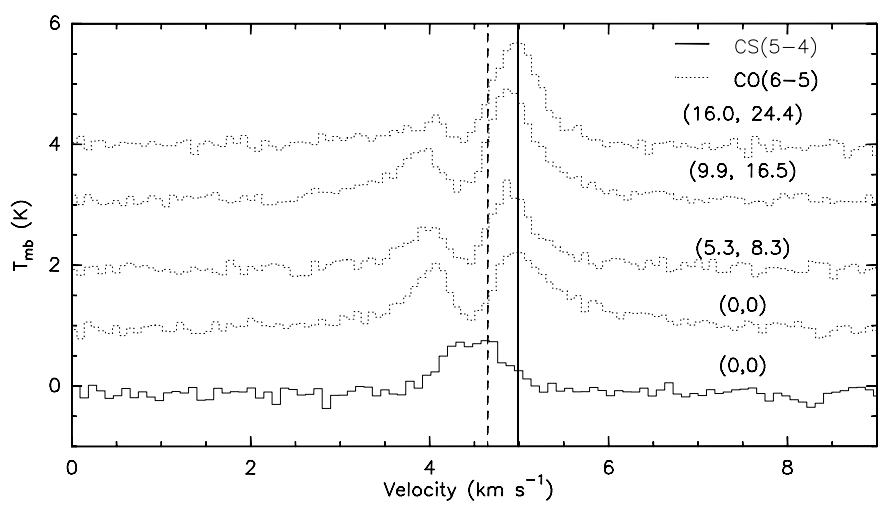

Fig. 21. Comparison of the CS 5-4 spectrum (solid) toward the central position of Cha-MMS1 and CO 6-5 spectra (dotted) taken at the central position and in the north-east direction toward the nearby Class I protostar. The offset position of each spectrum is given in arcseconds relative to the central position. The CO 6-5 spectra are shifted vertically by a step of $1 \mathrm{~K}$ for clarity. The solid vertical line roughly corresponds to the velocity of the CO 6-5 red peak, while the dashed line marks the velocity of the peak of the CS 5-4 excess emission.

the extended blueshifted CS 2-1 emission around Cha-MMS1. As recalled by Ladd et al. (2011), the HH objects 49 and 50, which are thought to be associated with this outflow, are redshifted. This suggests that the outflow axis lies close to the plane of the sky and, provided its opening angle is large enough, it could also explain the presence of extended, redshifted, wing emission in CS $2-1$ in the vicinity of Cha-MMS1. This emission is, however, difficult to disentangle from the ambient cloud emission in $\mathrm{CO} 3-2$, and it was excluded from the range of velocities used by Belloche et al. (2006) to produce their map of the redshifted outflow lobe.

\subsection{Interpretation of the $P-V$ diagrams}

Determining the nature of the velocity gradients in positionvelocity diagrams is not straightforward because many processes such as rotation, infall, outflow, and turbulence can influence their shape (e.g., Tobin et al. 2012; Burkert \& Bodenheimer 2000). Tobin et al. (2012) suggest that projected infall in filamentary protostellar envelopes can dominate the velocity gradients for scales larger than 1000 AU. Burkert \& Bodenheimer (2000) show that turbulence can produce velocity gradients that can be mistaken for rotation. In Sect. 3.3 we found no significant velocity gradient in the direction parallel to the filament in which Cha-MMS1 is embedded, but a significant one in the perpendicular direction. If we can approximate the filamentary geometry of the continuum emission as axisymmetric, and if infall plays a significant role in producing these velocity shifts, we would expect to see its contribution in the $P-V$ diagrams in the filament direction. That this is not the case enhances the probability that the velocity gradients we observe perpendicular to the filament are due to rotation.

The $P-V$ diagrams of $\mathrm{C}^{18} \mathrm{O} 2-1$ and $\mathrm{C}^{17} \mathrm{O} 2-1$ cover almost the same extent as the width of the filament $(\sim 0.1 \mathrm{pc})$. As the outermost positions of $\mathrm{C}^{18} \mathrm{O} 2-1$ and $\mathrm{C}^{17} \mathrm{O} 2-1$ reach the edges of the filament (see Fig. 1d), we cannot exclude the possibility that turbulent motions influence the shape of the velocity gradients on these scales. However, the "S" shape of the $\mathrm{C}^{18} \mathrm{O} 2-1$ and $\mathrm{C}^{17} \mathrm{O} 2-1 P-V$ curve at the outermost positions on either side of the central position is relatively symmetric (see Fig. 5c) and therefore, probably indicative of rotation rather than random turbulent motions. If the interpretation in terms of rotation is valid, then the " $\mathrm{S}$ " shape of the $P-V$ curve indicates that the filament is rotating in a differential manner, the outer parts $(r>8000 \mathrm{AU})$ rotating more slowly than the inner parts. The turn-over of the $\mathrm{C}^{18} \mathrm{O}$ and $\mathrm{C}^{17} \mathrm{O} P-V$ curves occurs very close to the edge of the filament as traced with LABOCA. We speculate that this behaviour is related to the formation process of the filament, but the physics of this process would have to be investigated.

We obtained a velocity gradient of $3.1 \pm 0.1 \mathrm{~km} \mathrm{~s}^{-1} \mathrm{pc}^{-1}$ over $r<8000 \mathrm{AU}$ for Cha-MMS1. This is similar to the velocity gradients often found in dense cores and attributed to rotation, with magnitude typically ranging from $\sim 0.3 \mathrm{~km} \mathrm{~s}^{-1} \mathrm{pc}^{-1}$ to $\sim 6 \mathrm{~km} \mathrm{~s}^{-1} \mathrm{pc}^{-1}$ on typical scales of $\sim 0.1 \mathrm{pc}$ (e.g., Goodman et al. 1993; Caselli et al. 2002; Belloche et al. 2002; Tafalla et al. 2004). The " $\mathrm{S}$ " shape of the $\mathrm{C}^{17} \mathrm{O} 2-1$ and $\mathrm{C}^{18} \mathrm{O} 2-1$ $P-V$ diagrams of Cha-MMS1 is very reminiscent of the $P-V$ diagrams of the young Class 0 protostar IRAM 04191 located in Taurus. Belloche et al. (2002) derive a rotational angular velocity of $9 \pm 3 \mathrm{~km} \mathrm{~s}^{-1} \mathrm{pc}^{-1}$ at a radius of $2800 \mathrm{AU}$ and $1.9 \pm$ $0.2 \mathrm{~km} \mathrm{~s}^{-1} \mathrm{pc}^{-1}$ at 7000 AU. They conclude that IRAM 04191 shows clear signs of differential rotation in the envelope. On scales of $\sim 1000 \mathrm{AU}$, the envelope rotates even faster (Belloche $\&$ André 2004). As mentioned in the previous paragraph, the $P-V$ diagram of Cha-MMS1 is also consistent with differential rotation, but a major difference compared to IRAM 04191 is that this concerns only the outer parts of the envelope ( $r>8000 \mathrm{AU})$. At smaller radii down to $\sim 4000 \mathrm{AU}$, the velocity profile is consistent with solid-body rotation. However, a puzzling feature of the $P-V$ diagrams of Cha-MMS1 is the even flatter velocity gradient in the inner parts of the envelope below $4000 \mathrm{AU}$, with an amplitude lower than $2 \mathrm{~km} \mathrm{~s}^{-1} \mathrm{pc}^{-1}$.

If the velocity gradients really trace rotation, then the envelope of Cha-MMS1 has a very peculiar rotational structure: the inner parts rotate more slowly than the bulk of the envelope, and the outer parts also rotate more slowly. A collapsing, magnetised core is expected to have an angular velocity increasing toward the centre (Basu \& Mouschovias 1995), the exact shape of the profile depending on the initial angular momentum distribution. Since we see evidence of infall motions in the envelope of Cha-MMS1, its peculiar rotational structure suggests that an efficient mechanism removing angular momentum during the collapse is at work over the range of radii $2000 \mathrm{AU}$ to $8000 \mathrm{AU}$. It would be interesting to investigate whether magnetic braking is efficient enough to account for this angular momentum removal in Cha-MMS1. Measuring the magnetic field structures and ionisation levels of the Cha-MMS1 and IRAM 04191 envelopes would then be necessary to compare the two sources and understand why they behave so differently in terms of rotation.

\subsubsection{Centrifugal acceleration and rotational energy}

If we interpret the velocity gradients in the $P-V$ diagrams as rotation, then we can estimate the dynamical importance of rotation for Cha-MMS1. We assume solid-body rotation for the inner envelope at $r<8000 \mathrm{AU}$ and an inclination of the rotation axis in the range $45^{\circ}-60^{\circ}$, as derived in Sect. 3.1. The angular velocity then ranges from $4.4 \mathrm{~km} \mathrm{~s}^{-1} \mathrm{pc}^{-1}$ to $3.6 \pm 0.1 \mathrm{~km} \mathrm{~s}^{-1} \mathrm{pc}^{-1}$. We compute the centrifugal acceleration and the local gravitational field as

$$
\begin{aligned}
a_{\mathrm{cen}} & =\frac{v_{\mathrm{rot}}^{2}}{r}=\Omega^{2} r, \\
g & =G \times \frac{M_{\mathrm{env}}+M_{\mathrm{obj}}}{r^{2}},
\end{aligned}
$$




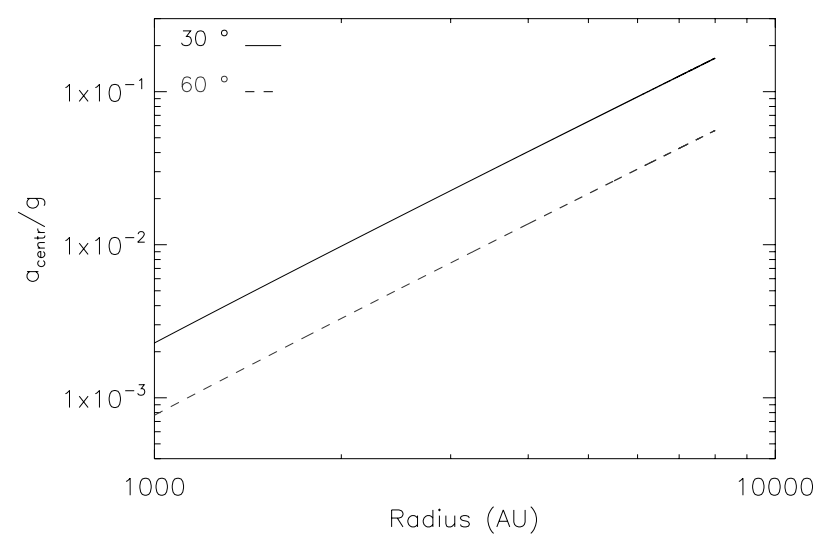

Fig. 22. Ratio of centrifugal to gravitational accelerations for the inner part of Cha-MMS1 that may be undergoing solid-body rotation. The upper and lower curves are for an inclination of $30^{\circ}$ and $60^{\circ}$, respectively.

where $a_{\text {cen }}, v_{\text {rot }}, \Omega, g, G, M_{\mathrm{env}}, M_{\mathrm{obj}}$, and $r$ are the centrifugal acceleration, the rotational velocity, the angular velocity, the gravitational acceleration, the gravitational constant, the envelope mass, the mass of the central object, and the radius, respectively. We assume that the envelope mass is proportional to the radius (density proportional to $r^{-2}$ ) and that a mass of $1.44 M_{\odot}$ is enclosed within a radius of $3750 \mathrm{AU}$, as derived from the LABOCA $870 \mu \mathrm{m}$ dust continuum map (Belloche et al. 2011a).

Figure 22 shows the variation of $a_{\text {cen }} / g$ as a function of radius. Within the framework of our assumptions, the centrifugal acceleration represents at most $20 \%$ of the gravitational acceleration. Thus rotation does not provide significant support to the envelope on scales of a few thousand AU. A similar conclusion was drawn by, say, Caselli et al. (2002) for their sample of dense cores, while Belloche et al. (2002) found that the centrifugal acceleration was a sizeable fraction of the gravitational acceleration on such scales in IRAM 04191 (up to 40\%).

The ratio of rotational kinetic energy to the core's gravitational energy for a centrally peaked $r^{-2}$ density profile, $\beta_{\text {rot }}$, is given by (Goodman et al. 1993):

$\beta_{\mathrm{rot}}=\frac{\Omega^{2} R^{3}}{9 G M}$

We obtain values of $\beta_{\text {rot }} \sim 0.02$ and $\sim 0.006$ at $8000 \mathrm{AU}$ for inclinations of $30^{\circ}$ and $60^{\circ}$ degrees, respectively.

\subsection{Implications of the infall velocity structure of Cha-MMS1}

In this section we compare the infall velocity distribution of Cha-MMS1 with predictions from theory and observations of other collapsing cores. The number of uncertainties involved is large, both in constraining the velocity profile of Cha-MMS1 from the radiative transfer modelling, as well as in the various existent theoretical collapse models in terms of initial and boundary conditions, and the inclusion or omission of either magnetic fields or rotation. Therefore, a comparison of our source with theoretical models is not straightforward and we only aim to qualitatively discuss which collapse models provide better agreement with the infall profile we derived for Cha-MMS1.

\subsubsection{Infall in Cha-MMS1 and in other observed cores}

Figure 20 shows the infall velocities consistent with the envelope of Cha-MMS1 as derived from the radiative transfer modelling in Sect. 4. We can discern two distinct regimes in terms of the envelope motions of Cha-MMS1, one below $3300 \mathrm{AU}$ and one within $3300 \mathrm{AU} \leq r \leq 30000 \mathrm{AU}$. At radii larger than $3300 \mathrm{AU}$ we constrain the velocity profile of Cha-MMS1 better (more tightly). The velocities are sub- to transonic within the range of $0.1 \mathrm{~km} \mathrm{~s}^{-1}-0.2 \mathrm{~km} \mathrm{~s}^{-1}$. At radii below $3300 \mathrm{AU}$, where the model degeneracies are greater, Cha-MMS1 is consistent both with increasing and decreasing central velocities, ranging from $0.04 \mathrm{~km} \mathrm{~s}^{-1}$ to $0.6 \mathrm{~km} \mathrm{~s}^{-1}$. Therefore, the inner $(r<3300 \mathrm{AU})$ envelope is characterised by velocities that can reach supersonic values compared to the outer $(r>3300 \mathrm{AU})$, more quiescent regions.

We also found that an inner, free-fall velocity distribution proportional to $r^{-0.5}$ is consistent with the envelope of Cha-MMS1 with an upper limit of $r \sim 9000$ AU. If we assume that the latter radius signifies the distance that the reflected expansion wave has so far covered while travelling outwards at the speed of sound, as described in the inside-out collapse scenario (Shu 1977), then we can estimate an upper limit to the age of Cha-MMS1 as $t \sim \frac{r_{\mathrm{s}}}{a_{\mathrm{s}}}=2 \times 10^{5}$ years or $0.2 \mathrm{Myr}$. The sound speed, $a_{\mathrm{S}} \sim 0.2 \mathrm{~km} \mathrm{~s}^{-1}$, is computed for a temperature of $9 \mathrm{~K}$ and assumes isothermality. In our models we have an isothermal temperature of $9 \mathrm{~K}$ only for $r>2000 \mathrm{AU}$, so this condition does not hold for the very inner radii. However, we only use the derived value as a crude estimate. Since this lifetime is an upper limit, it is consistent with both a first core (see Sect. 1.1 for FHSC lifetimes) or a Class 0 protostar. The prestellar phase lifetime is estimated to be $\sim 0.5 \mathrm{Myr}$ (Evans et al. 2009), and Class 0 objects have estimated lifetimes ranging from $\sim 4-9 \times 10^{4} \mathrm{yr}$ (Maury et al. 2011) to $\sim 1.6 \times 10^{5} \mathrm{yr}$ (Evans et al. 2009).

Belloche et al. (2002) similarly performed radiative transfer modelling of their molecular transitions towards the young Class 0 IRAM 04191 protostar using the MAPYSO code. They obtained an infall velocity of $0.15 \mathrm{~km} \mathrm{~s}^{-1}$ at a radius of $1500 \mathrm{AU}$ and $0.1 \mathrm{~km} \mathrm{~s}^{-1}$ up to $\sim 11000$ AU for their "best" fit. Their range of consistent infall velocities is similar to ours, albeit better constrained at small radii. For $r \geq 2000$ AU they find infall velocities in the range of $\sim 0.07$ and $\sim 0.15 \mathrm{~km} \mathrm{~s}^{-1}$, similar to the range $0.1 \mathrm{~km} \mathrm{~s}^{-1}-0.2 \mathrm{~km} \mathrm{~s}^{-1}$ that we obtain for Cha-MMS1 at $r \geq 3300$ AU. Nevertheless, they constrain the inner $r \leq$ 3000 AU velocity profile to that of a power law, whereas in the case of Cha-MMS1 power law, uniform and decreasing velocity distributions are consistent with our data below 3300 AU. Therefore, the infall velocity structures of both Cha-MMS1 and IRAM 04191 cover a similar range of velocities for $r>$ $3000 \mathrm{AU}$, with Cha-MMS1 being additionally consistent with a wider range of velocity profiles at small radii. The flat, or decreasing velocity distributions at the inner core of Cha-MMS1 could represent a younger evolutionary phase than that of a protostar, if central free-fall has not yet taken place on these scales. The age of IRAM 04191 was estimated to be $\leq 3 \times 10^{4} \mathrm{yr}$, assuming $2000 \mathrm{AU}$ is the radius below which there is a free fall velocity field structure, while we previously estimated an age of $\leq 2 \times 10^{5} \mathrm{yr}$ for Cha-MMS1.

The infall velocities we derive are mostly within the sub- to transonic range for $r \geq 3300$ AU ( $\geq 0.02$ pc). Lee et al. (1999) found subsonic inward velocities of $0.04 \mathrm{~km} \mathrm{~s}^{-1}-0.1 \mathrm{~km} \mathrm{~s}^{-1}$ for a sample of 17 starless cores showing evidence of infall motions. L1544 is one of the starless cores that has been extensively studied (e.g., Tafalla et al. 1998; Williams et al. 1999). These studies have shown that L1544 is undergoing inward motions with velocities up to $\sim 0.1 \mathrm{~km} \mathrm{~s}^{-1}$ over $\sim 0.1 \mathrm{pc}$. Such extended inward velocities imply that the velocity fields of Cha-MMS1, like that 
of L1544, are consistent with each other but inconsistent with the static SIS Shu (1977) model.

\subsubsection{Comparison to theoretical models}

In this section, we compare the infall velocity field derived for Cha-MMS1 with the velocities predicted by theoretical models. We resolve radii $r \geq 700 \mathrm{AU}$ from our observations and find an infall velocity range of $\sim 0.05 \mathrm{~km} \mathrm{~s}^{-1}-0.5 \mathrm{~km} \mathrm{~s}^{-1}$ for this radius $(r \sim 700 \mathrm{AU})$. We used 3D radiation-magneto-hydrodynamic models to extract the radial velocity structure for a $1 M_{\odot}$ (MU2 model; Commerçon et al. 2012) and $5 M_{\odot}$ dense core (Fig. 20). The mass estimate of Cha-MMS1 from continuum observations yields $1.44 M_{\odot}$ within a radius of $3750 \mathrm{AU}$ (Belloche et al. 2011a).

Both models have a normalised mass-to-magnetic-flux ratio of 2. They employ an azimuthal density perturbation to assist fragmentation. The ratio of thermal to gravitational energy is 0.37 for the $1 M_{\odot}$ model and 0.35 for the $5 M_{\odot}$ model, the ratio of rotational to gravitational energies 0.045 and $0.03(\sim 2$ and $\sim 1.5$ times greater than the upper limit of Cha-MMS1's $\beta_{\text {rot }}$, respectively), and the initial temperatures are $11 \mathrm{~K}$ and $10 \mathrm{~K}$, respectively. Their corresponding initial radii are $3300 \mathrm{AU}$ and $20000 \mathrm{AU}$. The density profile of the $1 M_{\odot}$ case is initially uniform, whereas it scales as $r^{-2}$ at the outer radii in the $5 M_{\odot}$ case. The $1 M_{\odot}$ and $5 M_{\odot}$ models are denser at the equatorial plane of the cores by a factor of $\sim 3$ and $\sim 4$, respectively, compared to the density profile we used for Cha-MMS1 in Sect. 4.

The models predict the evolution of the $24 \mu \mathrm{m}$ and $70 \mu \mathrm{m}$ flux densities during the first core phase. The 3D radiative transfer code RADMC-3D by Dullemond ${ }^{8}$ is used for the postprocessing of the RMHD calculations (see Commerçon et al. 2012 for more details). The flux densities of Cha-MMS1 are, within a factor of $\sim 2$, consistent with a first core age of $\sim 850 \mathrm{yr}$ based on the $1 M_{\odot}$ model, and $2680 \mathrm{yr}$ based on the $5 M_{\odot}$ model (see Sect. 3.1.2). The average radial velocity distribution of both first-core models close to the equatorial plane (to avoid outflow contamination) are shown in Fig. 20. We can only explore the velocity profile of the $1 M_{\odot}$ model for $r<2500 \mathrm{AU}$ due to its smaller initial radius (3300 AU). The radial velocities it predicts up to $\sim 2000 \mathrm{AU}$ are within the range of velocities we constrain for Cha-MMS1. For $r>2000$ AU the velocities decrease to zero as we approach the boundary of the core. The average velocity extracted from the $5 M_{\odot}$ model exhibits a velocity profile that follows the upper limit of Cha-MMS1's velocity range up to $\sim 8000 \mathrm{AU}$, and is within this range for $8000 \mathrm{AU}<r<12000 \mathrm{AU}$. The slightly higher velocities predicted by the model compared to our upper limit for Cha-MMS1 may be due to the higher densities of the model.The velocity dip that peaks at $\sim 18000 \mathrm{AU}$ in Fig. 20 is a remnant of a small initial expansion at the initial core radius of $\sim 20000 \mathrm{AU}$ that preceded the first core collapse, and therefore is of no physical importance for interpretating of the collapse process in the model compared to the observational constraints.

We now compare our results with other first core models that show reasonable agreement with the properties of Cha-MMS1. Masunaga et al. (1998) explored the protostellar collapse of a cloud core via radiation hydrodynamic simulations assuming spherical symmetry, and specifically focussed on the formation of the first hydrostatic core. For the typical case of $1 M_{\odot}$ and $10 \mathrm{~K}$ they find a homogeneous density structure inside the

\footnotetext{
8 http://www.ita.uni-heidelberg.de/ dullemond/ software/radmc-3d/
}

first core and an outer density distribution proportional to $r^{-2}$. The velocity field structure after the first core formation $(\rho \sim$ $10^{-13} \mathrm{~g} \mathrm{~cm}^{-3}$ ) does not change for scales larger than $\sim 1000 \mathrm{AU}$ for the different evolutionary times they present. They find supersonic velocities out to radii of $\sim 3000 \mathrm{AU}$, in very good agreement with the radius of $\sim 3300$ AU below which supersonic motions are compatible with the envelope of Cha-MMS1. The infall velocities at $1000 \mathrm{AU}, 3000 \mathrm{AU}$, and $9000 \mathrm{AU}$ they predict are $\sim 0.25 \mathrm{~km} \mathrm{~s}^{-1}, \sim 0.2 \mathrm{~km} \mathrm{~s}^{-1}$, and $\sim 0.1 \mathrm{~km} \mathrm{~s}^{-1}$. At the same radii we obtain velocity ranges of $0.06-0.5 \mathrm{~km} \mathrm{~s}^{-1}, 0.1-0.25 \mathrm{~km} \mathrm{~s}^{-1}$, and $0.1-0.2 \mathrm{~km} \mathrm{~s}^{-1}$. The velocity structure of Cha-MMS1 is therefore also consistent with the predictions of Masunaga et al. (1998) for a first core resulting from the collapse of a $1 M_{\odot}$ nonrotating, non-magnetised cloud.

Saigo et al. (2008) investigated the effect of different initial rotation speeds during protostellar collapse and the formation of the first and second hydrostatic cores by performing 3D hydrodynamical simulations of cores with slow, moderate, and fast initial rotation. For a slowly rotating cloud $\left(\beta_{\text {rot }} \sim 0.015-0.09\right.$, i.e., consistent with the respective ratio of Cha-MMS1), the infall velocity structure during the first core phase is described by supersonic motions below a radius of $\sim 3000 \mathrm{AU}$, and infall velocities of $\sim 0.4 \mathrm{~km} \mathrm{~s}^{-1}, \sim 0.3 \mathrm{~km} \mathrm{~s}^{-1}$, and $\sim 0.15 \mathrm{~km} \mathrm{~s}^{-1}$ at radii of $1000 \mathrm{AU}, 3000 \mathrm{AU}$, and $9000 \mathrm{AU}$. The infall velocity predictions of Saigo et al. (2008) for the model with slow initial rotation is therefore consistent with the velocity ranges of $0.06-0.5 \mathrm{~km} \mathrm{~s}^{-1}, 0.1-0.25 \mathrm{~km} \mathrm{~s}^{-1}$, and $0.1-0.2 \mathrm{~km} \mathrm{~s}^{-1}$ that we obtain for Cha-MMS1 at the same radial distances.

Finally, Ciolek \& Basu (2000) presented an ambipolar diffusion model incorporating the observational constraints and physical parameters previously derived for the protostellar core L1544. They presented a non-rotating, ambipolar diffusion model with a lower background magnetic field strength (initial mass-to-flux ratio 0.8 in units of the critical value), which could reproduce the extended observed infall velocities of L1544, and concluded that L1544 might be a supercritical core undergoing magnetically diluted collapse. Their model predicts infall velocities of $\sim 0.2 \mathrm{~km} \mathrm{~s}^{-1}$ and $\sim 0.15 \mathrm{~km} \mathrm{~s}^{-1}$ at late times (approaching the first core densities), at $3000 \mathrm{AU}$ and $9000 \mathrm{AU}$ from the supercritical core, respectively, which are consistent with the infall velocity field structure we derive for Cha-MMS1 (Fig. 20). The spatial scale for supersonic infall velocities they predict at late times is $\sim 2000 \mathrm{AU}$. The agreement between the velocities of the two cores, especially for the late-time evolution models (approaching first core densities), might be indicating that the initial conditions of the model for L1544 adequately describes the initial conditions of Cha-MMS1. Cha-MMS1 might therefore be undergoing collapse that is physically similar to that of the prestellar dense core L1544.

In general, the velocities of the RMHD model agree with the inner envelope $(r<2000 \mathrm{AU})$ of Cha-MMS1 for a $1 M_{\odot}$ collapsing dense core and for $r<12000 \mathrm{AU}$ for a $5 M_{\odot}$ dense core at the FHSC stage. In the innermost 2000 AU, the velocity predictions of both models "bracket" the upper limit of the observationally constrained velocities for Cha-MMS1, and for larger radii the $5 M_{\odot}$ predictions closely follow this upper limit. Non-magnetised, rotating, as well as non-rotating models for the first core phase (Saigo et al. 2008; Masunaga et al. 1998) also produce infall velocity structures that are consistent with the infall motions of Cha-MMS1 on scales of $\sim 10000 \mathrm{AU}$ $(\sim 0.05 \mathrm{pc})$. Consequently, the collapse motions in the envelope of Cha-MMS1 are consistent with first core predictions.

Since our observations are not sensitive to the very inner part of Cha-MMS1, where rapid infall velocity changes are expected, 
we cannot distinguish between an evolved prestellar core, a first core, and a young Class 0 object based on the kinematics alone. The $24 \mu \mathrm{m}$ and $70 \mu \mathrm{m}$ detections of Cha-MMS1 rule out its prestellar nature, and thus shift the dilemma between a first core and a young Class 0 protostar. This dilemma can be solved by detecting a slow, compact outflow stemming from Cha-MMS1 via interferometric studies, which would differentiate between the two evolutionary phases. In any case, our kinematic and dynamical study has so far shown that its properties do not contradict the first-core predictions, and it is an interesting target for exploring the early protostellar stages of star-formation.

\section{Summary and conclusions}

We performed observations of the dense core Cha-MMS1 in various molecular transitions and conducted an analysis of the kinematics within the core in order to investigate its physical properties and dynamical state. We utilised a $1 \mathrm{D}$ radiative transfer code to constrain the infall velocity structure of the core. Our conclusions can be summarised as follows.

1. The internal luminosity of Cha-MMS1 is estimated from the predicted inclination-dependent time evolution of SEDs for the first core phase for a $1 M_{\odot}($ Commerçon et al. 2012) and a $5 M_{\odot}$ model. The $24 \mu \mathrm{m}$ and $70 \mu \mathrm{m}$ flux densities imply inclinations within the range of $30^{\circ} \leq i<60^{\circ}$ and an internal luminosity range of $0.08 L_{\odot}-0.18 L_{\odot}$.

2. The classical infall signature is detected in optically thick transitions, suggesting that the envelope of Cha-MMS1 is undergoing inward motions.

3. The position-velocity diagrams of optically thin transitions show velocity gradients perpendicular to the filament in which Cha-MMS1 is embedded. The average gradient over an extent of $\sim 16000 \mathrm{AU}$ in diameter is $3.1 \pm$ $0.1 \mathrm{~km} \mathrm{~s}^{-1} \mathrm{pc}^{-1}$, while we found no significant gradient along the filament. Interpreted in terms of rotation, these velocity variations imply solid-body rotation in the envelope up to a radius of $\sim 8000 \mathrm{AU}$, and slower, differential rotation from $\sim 8000$ AU to $\sim 12500 \mathrm{AU}$. The average velocity gradient in the range $2000 \mathrm{AU}-4000 \mathrm{AU}$ is surprisingly flatter, which is difficult to understand in terms of rotation.

4. The turbulent velocity dispersion in the core is uniform within a radius of $r \sim 5000 \mathrm{AU}$ parallel to the filament and $\sim 12500$ AU perpendicular to the filament. The nonthermal dispersion is similar to the mean thermal dispersion at a temperature of $9 \mathrm{~K}$, therefore implying an equipartition between thermal and non-thermal motions.

5. Our radiative transfer modelling yields subsonic to transonic infall velocities in the range $0.1 \mathrm{~km} \mathrm{~s}^{-1}-0.2 \mathrm{~km} \mathrm{~s}^{-1}$ for $3300 \mathrm{AU}<r<30000 \mathrm{AU}$. The velocity field is less well constrained in the inner parts for $r<3300$ AU. A velocity increasing as $r^{-0.5}$ toward the centre is consistent with the data, but we cannot exclude a decrease either. We find subsonic to supersonic velocities in the range $0.04 \mathrm{~km} \mathrm{~s}^{-1}-0.6 \mathrm{~km} \mathrm{~s}^{-1}$ for $r \leq 3300 \mathrm{AU}$.

6. Part of the redshifted emission of the high-density tracers CS 5-4, CO 7-6, and CO 6-5 is not reproduced by the radiative transfer model. This excess emission may indicate the presence of unresolved, higher velocity material at the inner core originating in a compact outflow driven by Cha-MMS1, or alternatively, it could arise due to contamination from the outflow of the nearby Class I protostar.
7. We find relatively good agreement between the infall velocity profile derived for Cha-MMS1 and predictions of 3D RMHD simulations for the first hydrostatic core phase.

Both the kinematic agreement with the predictions of RMHD simulations and the possible presence of a compact outflow suggested above are consistent with Cha-MMS1 being at the stage of the first hydrostatic core. However, we cannot affirm the object's nature without high-resolution interferometric observations to search for and image a compact, slow outflow. With the early prestellar core phase ruled out due to the object's $24 \mu \mathrm{m}$ and $70 \mu \mathrm{m}$ detection, Cha-MMS1 is either a first core or a young Class 0 protostar, and our kinematical study cannot exclude either possibility.

Acknowledgements. We thank Bérengère Parise, Philippe André, and Tyler Bourke for their insightful comments and suggestions, the APEX and Mopra staff for their support during the observations, and the referee for the feedback that helped in improving the quality of this paper. AET was supported for this research through a stipend from the International Max Planck Research School (IMPRS) for Astronomy and Astrophysics at the Universities of Bonn and Cologne.

\section{References}

Alves, J. F., Lada, C. J., \& Lada, E. A. 2001, Nature, 409, 159

André, P., Ward-Thompson, D., \& Barsony, M. 1993, ApJ, 406, 122

André, P., Ward-Thompson, D., \& Barsony, M. 2000, in Protostars and Planets IV (Arizona: University of Arizona Press), 59

André, P., Belloche, A., Motte, F., \& Peretto, N. 2007, A\&A, 472, 519

André, P., Basu, S., \& Inutsuka, S. 2009, in The formation and evolution of prestellar cores, ed. G. Chabrier (Cambridge University Press), 254

André, P., Men'shchikov, A., Bontemps, S., et al. 2010, A\&A, 518, L102

Arzoumanian, D., André, P., Didelon, P., et al. 2011, A\&A, 529, L6

Bacmann, A., André, P., Puget, J.-L., et al. 2000, A\&A, 361, 555

Bacmann, A., Lefloch, B., Ceccarelli, C., et al. 2002, A\&A, 389, L6

Barranco, J. A., \& Goodman, A. A. 1998, ApJ, 504, 207

Basu, S., \& Mouschovias, T. C. 1994, ApJ, 432, 720

Basu, S., \& Mouschovias, T. C. 1995, ApJ, 452, 386

Bate, M. R. 2011, MNRAS, 417, 2036

Belloche, A., \& André, P. 2004, A\&A, 419, L35

Belloche, A., André, P., Despois, D., \& Blinder, S. 2002, A\&A, 393, 927

Belloche, A., Parise, B., van der Tak, F. F. S., et al. 2006, A\&A, 454, L51

Belloche, A., Schuller, F., Parise, B., et al. 2011a, A\&A, 527, A145

Belloche, A., Parise, B., Schuller, F., et al. 2011b, A\&A, 535, A2

Benson, P. J., \& Myers, P. C. 1983, ApJ, 270, 589

Bergin, E. A., Ciardi, D. R., Lada, C. J., Alves, J., \& Lada, E. A. 2001, ApJ, 557, 209

Blinder, S. 1997, Ph.D. Thesis, Université de Bordeaux I

Bodenheimer, P., \& Sweigart, A. 1968, ApJ, 152, 515

Bourke, T. L., Myers, P. C., Evans, II, N. J., et al. 2006, ApJ, 649, L37

Burkert, A., \& Bodenheimer, P. 2000, ApJ, 543, 822

Cabrit, S., \& Bertout, C. 1990, ApJ, 348, 530

Caselli, P., Benson, P. J., Myers, P. C., \& Tafalla, M. 2002, ApJ, 572, 238

Chen, X., Arce, H. G., Zhang, Q., et al. 2010, ApJ, 715, 1344

Chen, X., Arce, H. G., Dunham, M. M., et al. 2012, ApJ, 751, 89

Christie, H., Viti, S., Yates, J., et al. 2012, MNRAS, 422, 968

Ciolek, G. E., \& Basu, S. 2000, ApJ, 529, 925

Commerçon, B., Hennebelle, P., Audit, E., Chabrier, G., \& Teyssier, R. 2010, A\&A, 510, L3

Commerçon, B., Launhardt, R., Dullemond, C., \& Henning, T. 2012, A\&A, 545, A98

Crapsi, A., Caselli, P., Walmsley, C. M., et al. 2004, A\&A, 420, 957

di Francesco, J., Evans, II, N. J., Caselli, P., et al. 2007, in Protostars and Planets V (Arizona: University of Arizona Press), 17

Dunham, M. M., Crapsi, A., Evans, II, N. J., et al. 2008, ApJS, 179, 249

Dunham, M. M., Chen, X., Arce, H. G., et al. 2011, ApJ, 742, 1

Enoch, M. L., Lee, J.-E., Harvey, P., Dunham, M. M., \& Schnee, S. 2010, ApJ, 722, L33

Evans, II, N. J., Rawlings, J. M. C., Shirley, Y. L., \& Mundy, L. G. 2001, ApJ, 557,193

Evans, II, N. J., Allen, L. E., Blake, G. A., et al. 2003, PASP, 115, 965

Evans, II, N. J., Dunham, M. M., Jørgensen, J. K., et al. 2009, ApJS, 181, 321 
A. E. Tsitali et al.: The dynamical state of the first hydrostatic core candidate Chamaeleon-MMS1

Fiedler, R. A., \& Mouschovias, T. C. 1993, ApJ, 415, 680

Foster, P. N., \& Chevalier, R. A. 1993, ApJ, 416, 303

Frerking, M. A., Wilson, R. W., Linke, R. A., \& Wannier, P. G. 1980, ApJ, 240, 65

Furuya, K., Aikawa, Y., Tomida, K., et al. 2012, ApJ, 758, 86

Goodman, A. A., Benson, P. J., Fuller, G. A., \& Myers, P. C. 1993, ApJ, 406, 528

Goodman, A. A., Barranco, J. A., Wilner, D. J., \& Heyer, M. H. 1998, ApJ, 504, 223

Haikala, L. K., Harju, J., Mattila, K., \& Toriseva, M. 2005, A\&A, 431, 149 Hennebelle, P., \& Fromang, S. 2008, A\&A, 477, 9

Hill, T., Motte, F., Didelon, P., et al. 2011, A\&A, 533, A94

Hiramatsu, M., Hayakawa, T., Tatematsu, K., et al. 2007, ApJ, 664, 964

Hunter, C. 1977, ApJ, 218, 834

Knude, J., \& Høg, E. 1998, A\&A, 338, 897

Könyves, V., André, P., Men'shchikov, A., et al. 2010, A\&A, 518, L106

Ladd, E. F., Wong, T., Bourke, T. L., \& Thompson, K. L. 2011, ApJ, 743, 108

Larson, R. B. 1969, MNRAS, 145, 271

Larson, R. B. 1981, MNRAS, 194, 809

Lee, C. W., Myers, P. C., \& Tafalla, M. 1999, ApJ, 526, 788

Lehtinen, K., Haikala, L. K., Mattila, K., \& Lemke, D. 2001, A\&A, 367, 311

Lehtinen, K., Harju, J., Kontinen, S., \& Higdon, J. L. 2003, A\&A, 401, 1017

Lesaffre, P., Belloche, A., Chièze, J.-P., \& André, P. 2005, A\&A, 443, 961

Machida, M. N., \& Matsumoto, T. 2011, MNRAS, 413, 2767

Machida, M. N., Inutsuka, S.-I., \& Matsumoto, T. 2008, ApJ, 676, 1088

Masunaga, H., \& Inutsuka, S.-I. 1999, ApJ, 510, 822

Masunaga, H., Miyama, S. M., \& Inutsuka, S.-I. 1998, ApJ, 495, 346

Maury, A. J., André, P., Men'shchikov, A., Könyves, V., \& Bontemps, S. 2011, A\&A, 535, A77

Men'shchikov, A., André, P., Didelon, P., et al. 2010, A\&A, 518, L103

Molinari, S., Swinyard, B., Bally, J., et al. 2010, A\&A, 518, L100

Motte, F., \& André, P. 2001, A\&A, 365, 440

Motte, F., Andre, P., \& Neri, R. 1998, A\&A, 336, 150

Nielbock, M., Launhardt, R., Steinacker, J., et al. 2012, A\&A, 547, A11
Omukai, K. 2007, PASJ, 59, 589

Penston, M. V. 1966, Roy. Greenwich Obs. Bull., 117, 299

Penston, M. V. 1969, MNRAS, 144, 425

Pezzuto, S., Elia, D., Schisano, E., et al. 2012, A\&A, 547, A54

Pilbratt, G. L., Riedinger, J. R., Passvogel, T., et al. 2010, A\&A, 518, L1

Pineda, J. E., Arce, H. G., Schnee, S., et al. 2011, ApJ, 743, 201

Price, D. J., Tricco, T. S., \& Bate, M. R. 2012, MNRAS, 423, L45

Rathborne, J. M., Lada, C. J., Muench, A. A., et al. 2009, ApJ, 699, 742

Reipurth, B., Nyman, L.-A., \& Chini, R. 1996, A\&A, 314, 258

Saigo, K., \& Tomisaka, K. 2011, ApJ, 728, 78

Saigo, K., Tomisaka, K., \& Matsumoto, T. 2008, ApJ, 674, 997

Shinnaga, H., Phillips, T. G., Furuya, R. S., \& Kitamura, Y. 2009, ApJ, 706, L226

Shu, F. H. 1977, ApJ, 214, 488

Stahler, S. W., \& Yen, J. J. 2010, MNRAS, 407, 2434

Tafalla, M., Mardones, D., Myers, P. C., et al. 1998, ApJ, 504, 900

Tafalla, M., Myers, P. C., Caselli, P., Walmsley, C. M., \& Comito, C. 2002, ApJ, 569,815

Tafalla, M., Myers, P. C., Caselli, P., \& Walmsley, C. M. 2004, A\&A, 416, 191

Takano, S., Masuda, A., Hirahara, Y., et al. 1998, A\&A, 329, 1156

Tassis, K., \& Mouschovias, T. C. 2007, ApJ, 660, 388

Terebey, S., Chandler, C. J., \& André, P. 1993, ApJ, 414, 759

Tobin, J. J., Hartmann, L., Bergin, E., et al. 2012, ApJ, 748, 16

Tomida, K., Machida, M. N., Saigo, K., Tomisaka, K., \& Matsumoto, T. 2010, ApJ, 725, L239

Tomisaka, K. 2002, ApJ, 575, 306

Tomisaka, K., \& Tomida, K. 2011, PASJ, 63, 1151

Walker, C. K., Lada, C. J., Young, E. T., Maloney, P. R., \& Wilking, B. A. 1986, ApJ, 309, L47

Whittet, D. C. B., Prusti, T., Franco, G. A. P., et al. 1997, A\&A, 327, 1194

Williams, J. P., Myers, P. C., Wilner, D. J., \& di Francesco, J. 1999, ApJ, 513, L61

Wilson, T. L., \& Rood, R. 1994, ARA\&A, 32, 191

Wouterloot, J. G. A., Brand, J., \& Henkel, C. 2005, A\&A, 430, 549

Zhou, S. 1992, ApJ, 394, 204 
Table 6. Mopra centroid velocities toward the central position, P1.

\begin{tabular}{lc}
\hline \hline Transition & $\begin{array}{c}V_{\mathrm{LSR}^{b}} \\
\left(\mathrm{~km} \mathrm{~s}^{-1}\right)\end{array}$ \\
\hline $\mathrm{HN}^{13} \mathrm{C} 1-0$ & $4.37 \pm 0.01$ \\
$\mathrm{HNCO} 4_{0,4}-3_{0,3}$ & $4.36 \pm 0.02$ \\
$\mathrm{c}-\mathrm{C}_{3} \mathrm{H}_{2} 3_{2,2}-3_{1,3}$ & $4.35 \pm 0.03$ \\
$\mathrm{HC}^{18} \mathrm{O}^{+} 1-0$ & $4.39 \pm 0.03$ \\
$\mathrm{H}^{13} \mathrm{CO}^{+} 1-0$ & $4.32 \pm 0.01$ \\
\hline
\end{tabular}

Notes. ${ }^{(a)}$ Only the central position was observed for each of these transitions. ${ }^{(b)}$ The correction of $0.1 \mathrm{~km} \mathrm{~s}^{-1}$ discussed in Sect. 3.2.1 has not been applied.

Table 7. CS 5-4 and $\mathrm{H}^{13} \mathrm{CO}^{+} 4-3$ centroid velocities (APEX) at positions perpendicular to the filament.

\begin{tabular}{lccccc}
\hline \hline Position $^{a}$ & $\Delta \alpha^{b}$ & $\Delta \delta^{b}$ & Spacing $^{c}$ & \multicolumn{2}{c}{$V_{\mathrm{LSR}}\left(\mathrm{km} \mathrm{s}^{-1}\right)$} \\
& $\left({ }^{\prime \prime}\right)$ & $\left(^{\prime \prime}\right)$ & $\left({ }^{\prime \prime}\right)$ & $\mathrm{CS} \mathrm{5-4}$ & $\mathrm{H}^{13} \mathrm{CO}^{+} 4-3$ \\
\hline $\mathrm{P} 1$ & 0 & 0 & 0 & $4.50 \pm 0.02$ & $4.43 \pm 0.02$ \\
$\mathrm{P}_{b} 2$ & -6.5 & 9.3 & 11.4 & $4.42 \pm 0.02$ & $4.41 \pm 0.02$ \\
$\mathrm{P}_{b} 3$ & -13.1 & 18.7 & 22.8 & $4.40 \pm 0.01$ & $4.44 \pm 0.06$ \\
$\mathrm{P}_{b} 4$ & 6.5 & -9.3 & 11.4 & $4.43 \pm 0.02$ & $4.42 \pm 0.04$ \\
$\mathrm{P}_{b} 5$ & 13.1 & -18.7 & 22.8 & $4.37 \pm 0.02$ & $4.65 \pm 0.31$ \\
\hline
\end{tabular}

Notes. ${ }^{(a)}$ These positions are shown in Fig. 1d. ${ }^{(b)}$ J2000 equatorial offset relative to the central position. ${ }^{(c)}$ Angular distance to the central position.

Table 8. $\mathrm{H}^{13} \mathrm{CO}^{+} 3-2$ centroid velocities (APEX) at positions parallel and perpendicular to the filament.

\begin{tabular}{lcccc}
\hline \hline Position $^{a}$ & $\begin{array}{c}\Delta \alpha^{b} \\
\left({ }^{\prime \prime}\right)\end{array}$ & $\begin{array}{c}\Delta \delta^{b} \\
\left({ }^{\prime \prime}\right)\end{array}$ & $\begin{array}{c}\text { Spacing }^{c} \\
\left({ }^{\prime \prime}\right)\end{array}$ & $\begin{array}{c}V_{\mathrm{LSR}}\left(\mathrm{km} \mathrm{s}^{-1}\right) \\
\mathrm{H}^{13} \mathrm{CO}^{+} 3-2\end{array}$ \\
\hline $\mathrm{P} 1$ & 0 & 0 & 0 & $4.41 \pm 0.02$ \\
perpendicular to the filament & & \\
$\mathrm{P}_{a} 2$ & -6.5 & 9.3 & 11.4 & $4.40 \pm 0.01$ \\
$\mathrm{P}_{a} 3$ & -13.1 & 18.7 & 22.8 & $4.41 \pm 0.02$ \\
$\mathrm{P}_{a} 4$ & -19.6 & 28.0 & 34.2 & $4.38 \pm 0.02$ \\
$\mathrm{P}_{a} 5$ & 6.5 & -9.3 & 11.4 & $4.36 \pm 0.04$ \\
$\mathrm{P}_{a} 6$ & 13.1 & -18.7 & 22.8 & $4.44 \pm 0.03$ \\
parallel to the filament & & & \\
$\mathrm{P}_{a} 8$ & 9.3 & 6.5 & 11.4 & $4.35 \pm 0.02$ \\
$\mathrm{P}_{a} 9$ & 18.7 & 13.1 & 22.8 & $4.32 \pm 0.04$ \\
$\mathrm{P}_{a} 10$ & 28.0 & 19.6 & 34.2 & $4.41 \pm 0.03$ \\
$\mathrm{P}_{a} 11$ & -9.3 & -6.5 & 11.4 & $4.41 \pm 0.02$ \\
$\mathrm{P}_{a} 12$ & -18.7 & -13.1 & 22.8 & $4.44 \pm 0.02$ \\
$\mathrm{P}_{a} 13$ & -28.0 & -19.6 & 34.2 & $4.43 \pm 0.02$ \\
\hline
\end{tabular}

Notes. ${ }^{(a)}$ These positions are shown in Fig. 1c. ${ }^{(b, c)}$ Same as Table 7.
Table 9. $\mathrm{C}^{17} \mathrm{O} 2-1$ and $\mathrm{C}^{18} \mathrm{O} 2-1$ centroid velocities (APEX) at positions perpendicular to the filament.

\begin{tabular}{lccccc}
\hline \hline Position $^{a}$ & $\Delta \alpha^{b}$ & $\Delta \delta^{b}$ & Spacing $^{c}$ & \multicolumn{2}{c}{$V_{\mathrm{LSR}}\left(\mathrm{km} \mathrm{s}^{-1}\right)$} \\
& $\left({ }^{\prime \prime}\right)$ & $\left({ }^{\prime \prime}\right)$ & $\left({ }^{\prime \prime}\right)$ & $\mathrm{C}^{17} \mathrm{O} 2-1$ & $\mathrm{C}^{18} \mathrm{O} 2-1$ \\
\hline $\mathrm{P} 1$ & 0 & 0 & 0 & $4.45 \pm 0.01$ & $4.47 \pm 0.01$ \\
$\mathrm{P}_{c} 2$ & -8 & 11.5 & 14.0 & $4.42 \pm 0.02$ & $4.45 \pm 0.01$ \\
$\mathrm{P}_{c} 3$ & -16 & 23 & 28.0 & $4.37 \pm 0.02$ & $4.40 \pm 0.01$ \\
$\mathrm{P}_{c} 4$ & -24 & 34.5 & 42.0 & $4.30 \pm 0.03$ & $4.32 \pm 0.01$ \\
$\mathrm{P}_{c} 5$ & -32 & 46 & 56.0 & $4.23 \pm 0.02$ & $4.28 \pm 0.01$ \\
$\mathrm{P}_{c} 6$ & -40 & 57.5 & 70.0 & $4.22 \pm 0.02$ & $4.27 \pm 0.01$ \\
$\mathrm{P}_{c} 7$ & -48 & 69 & 84.1 & $4.38 \pm 0.03$ & $4.33 \pm 0.02$ \\
$\mathrm{P}_{c} 8$ & 8 & -11.5 & 14.0 & $4.44 \pm 0.02$ & $4.48 \pm 0.01$ \\
$\mathrm{P}_{c} 9$ & 16 & -23 & 28.0 & $4.47 \pm 0.03$ & $4.51 \pm 0.01$ \\
$\mathrm{P}_{c} 10$ & 24 & -34.5 & 42.0 & $4.53 \pm 0.03$ & $4.52 \pm 0.01$ \\
$\mathrm{P}_{c} 11$ & 32 & -46 & 56.0 & $4.56 \pm 0.03$ & $4.55 \pm 0.01$ \\
$\mathrm{P}_{c} 12$ & 40 & -57.5 & 70.0 & $4.55 \pm 0.03$ & $4.51 \pm 0.01$ \\
$\mathrm{P}_{c} 13$ & 48 & -69 & 84.1 & $4.50 \pm 0.04$ & $4.52 \pm 0.02$ \\
\hline
\end{tabular}

Notes. ${ }^{(a)}$ These positions are shown in Fig. 1e. ${ }^{(b, c)}$ Same as Table 7.

Table 11. Observed linewidth, thermal, and non-thermal velocity dispersions toward the central position.

\begin{tabular}{lcccc}
\hline \hline Line & $\begin{array}{c}F W H M^{a} \\
\left(\mathrm{~km} \mathrm{~s}^{-1}\right)\end{array}$ & $\begin{array}{c}\sigma_{\mathrm{th}^{b}}{ }^{b} \\
\left.\mathrm{~km} \mathrm{~s}^{-1}\right)\end{array}$ & $\begin{array}{c}\sigma_{\text {nth }}{ }^{c} \\
\left(\mathrm{~km} \mathrm{~s}^{-1}\right)\end{array}$ & $\frac{\sigma_{\text {thth }}{ }^{d}}{}$ \\
\hline $\mathrm{C}^{34} \mathrm{~S} 2-1$ & $0.57 \pm 0.05$ & 0.04 & $0.24 \pm 0.02$ & $1.33 \pm 0.11$ \\
$\mathrm{HC}_{3} \mathrm{~N} \mathrm{10-9}$ & $0.50 \pm 0.009$ & 0.04 & $0.21 \pm 0.006$ & $1.17 \pm 0.03$ \\
$\mathrm{~N}_{2} \mathrm{H}^{+} 1-0^{e}$ & $0.48 \pm 0.002$ & 0.05 & $0.20 \pm 0.001$ & $1.11 \pm 0.006$ \\
$\mathrm{CH}_{3} \mathrm{OH}-A 2_{0,2}-1_{0,1}$ & $0.48 \pm 0.01$ & 0.05 & $0.20 \pm 0.007$ & $1.11 \pm 0.04$ \\
$\mathrm{CH}_{3} \mathrm{OH}-E 2_{1,2}-1_{1,1}$ & $0.47 \pm 0.02$ & 0.05 & $0.19 \pm 0.01$ & $1.06 \pm 0.06$ \\
$\mathrm{H}^{13} \mathrm{CO}^{+} 3-2$ & $0.55 \pm 0.03$ & 0.05 & $0.23 \pm 0.02$ & $1.28 \pm 0.11$ \\
$\mathrm{H}^{13} \mathrm{CO}^{+} 4-3$ & $0.48 \pm 0.04$ & 0.05 & $0.20 \pm 0.03$ & $1.11 \pm 0.17$ \\
$\mathrm{C}^{17} \mathrm{O} 2-1$ & $0.50 \pm 0.04$ & 0.05 & $0.20 \pm 0.02$ & $1.11 \pm 0.11$ \\
$\mathrm{C}^{18} \mathrm{O} 2-1$ & $0.62 \pm 0.02$ & 0.05 & $0.26 \pm 0.008$ & $1.44 \pm 0.04$ \\
$\mathrm{CS}_{5} 5-4$ & $0.85 \pm 0.04$ & 0.04 & $0.36 \pm 0.03$ & $2.00 \pm 0.17$ \\
$\mathrm{HN}{ }^{13} \mathrm{C} 1-0$ & $0.42 \pm 0.03$ & 0.05 & $0.17 \pm 0.01$ & $0.94 \pm 0.06$ \\
$\mathrm{HNCO} \mathrm{4} 4_{0,4}-3_{0,3}$ & $0.54 \pm 0.03$ & 0.04 & $0.22 \pm 0.01$ & $1.22 \pm 0.06$ \\
$\mathrm{HC} C^{18} \mathrm{O}^{+} 1-0$ & $0.50 \pm 0.06$ & 0.05 & $0.21 \pm 0.03$ & $1.17 \pm 0.17$ \\
$\mathrm{c}-\mathrm{C}_{3} \mathrm{H}_{2} 3_{2,2}-3_{1,3}$ & $0.52 \pm 0.08$ & 0.04 & $0.22 \pm 0.03$ & $1.22 \pm 0.17$ \\
\hline
\end{tabular}

Notes. ${ }^{(a)}$ Observed linewidth deduced from Gaussian or hyperfine fits to the spectra. ${ }^{(b)}$ Thermal dispersion computed with Eq. (2). ${ }^{(c)}$ Nonthermal dispersion computed using Eq. (3). (d) Ratio of non-thermal to mean thermal dispersion, with $\mu=2.37$. ${ }^{(e)}$ These transitions have a hyperfine structure and were fitted with the "HFS" fitting method in CLASS. The other transitions were fitted using the "GAUSS" method. 
A. E. Tsitali et al.: The dynamical state of the first hydrostatic core candidate Chamaeleon-MMS1
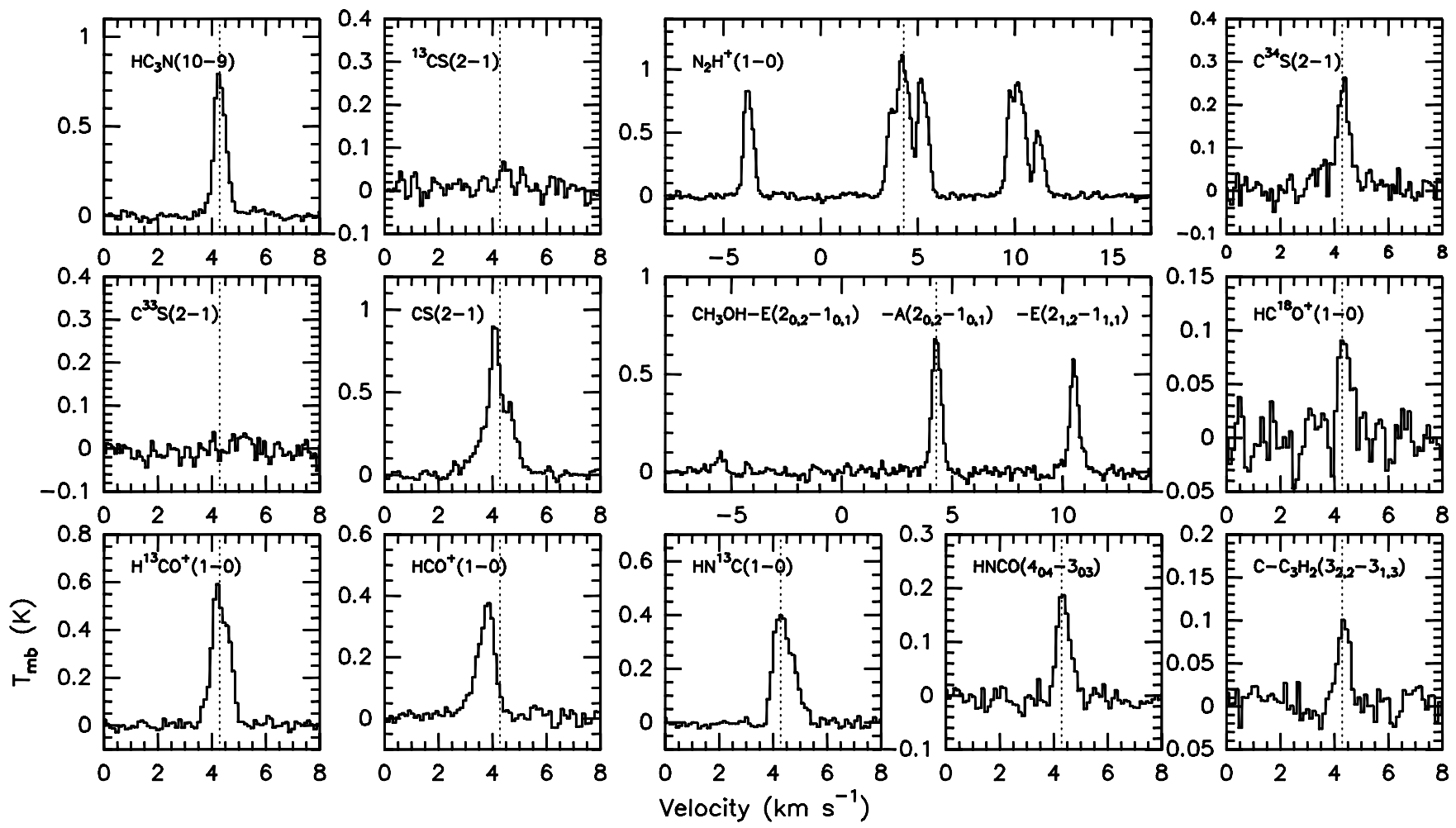

Fig. 2. Transitions observed with Mopra towards the central position of Cha-MMS1, in main-beam brightness temperature scale. The dotted line shows the systemic velocity of Cha-MMS1, derived from a seven component hyperfine fit to the $\mathrm{N}_{2} \mathrm{H}^{+} 1-0$ multiplet.

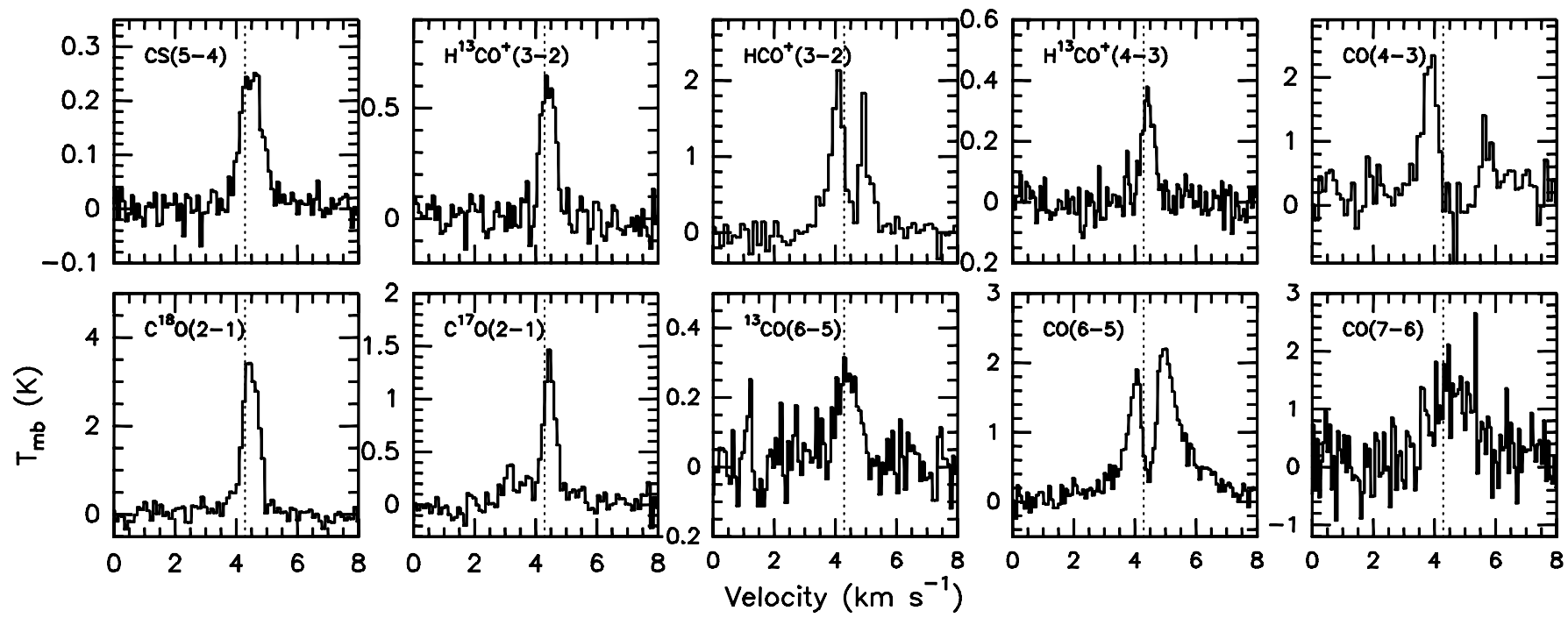

Fig. 3. Transitions observed with APEX towards the central position of Cha-MMS1, in main-beam brightness temperature scale. The dotted line shows the systemic velocity of Cha-MMS1, derived from a seven component hyperfine fit to the $\mathrm{N}_{2} \mathrm{H}^{+} 1-0$ multiplet observed with Mopra without correcting for the $+0.1 \mathrm{~km} \mathrm{~s}^{-1}$ velocity shift (see Sect. 3.2.1). 
A\&A 557, A98 (2013)

$0^{\prime \prime} \quad 17.5^{\prime \prime} \quad 35^{\prime \prime} \quad 52.5^{\prime \prime}$
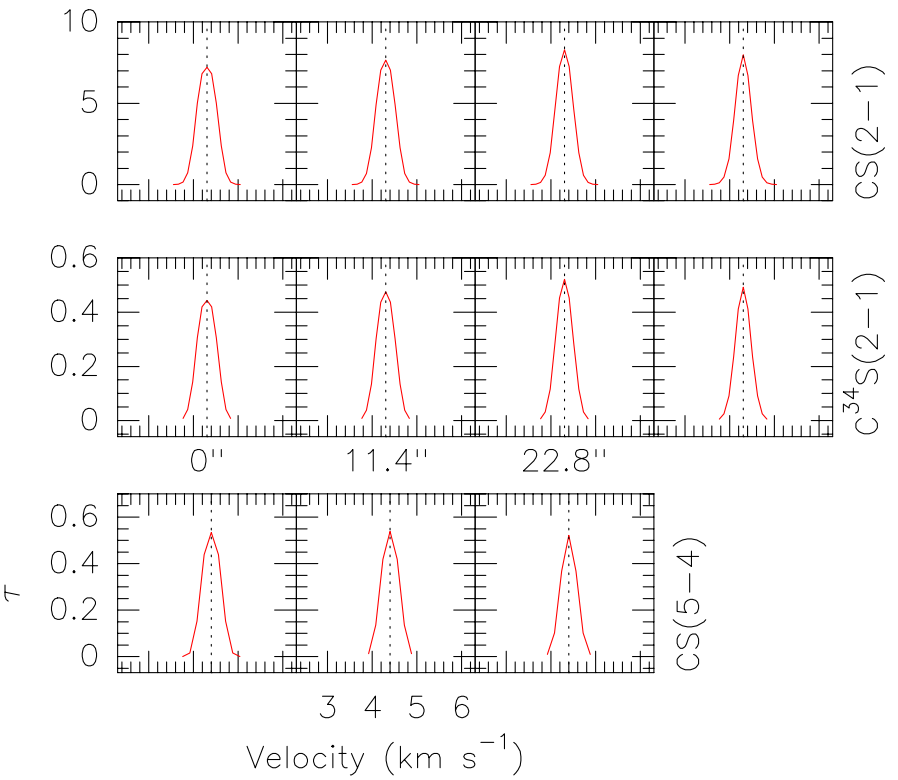

Fig. 12. Transition opacities for the best fit model "MCS". The dotted line shows the systemic velocity of Cha-MMS1. For the APEX spectra, a correction of $0.1 \mathrm{~km} \mathrm{~s}^{-1}$ was added (see Sect. 3.2.1).

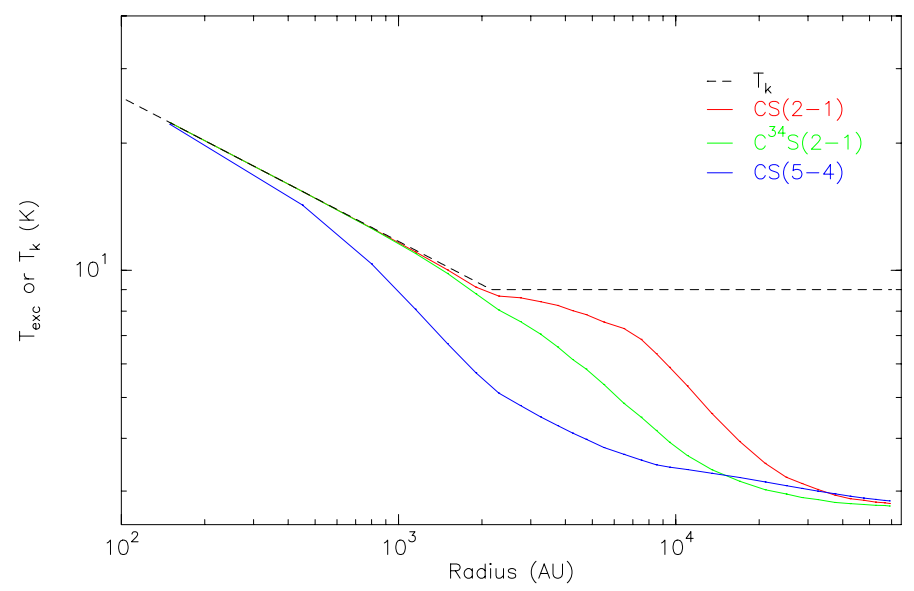

Fig. 13. Kinetic (black) and excitation temperature (colour) for all the transitions of the best fit model "MCS".
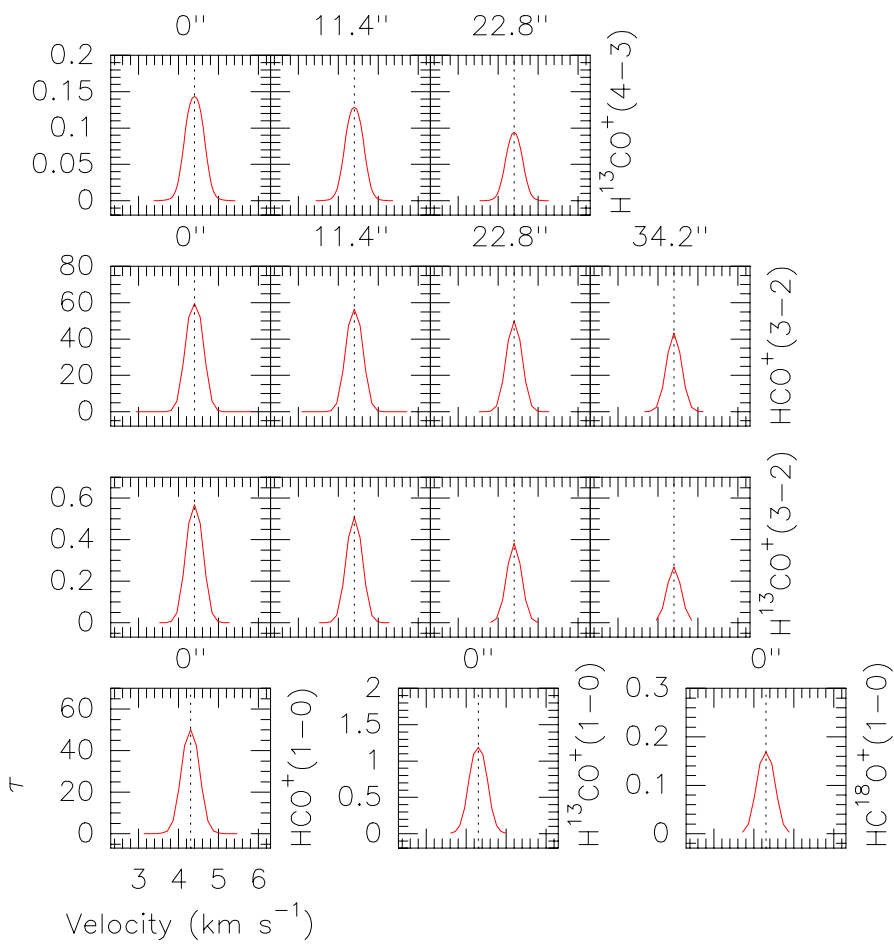

Fig. 15. Transition opacities for the best fit model "MHCOP". The dotted line shows the systemic velocity of Cha-MMS1. For the APEX spectra, a correction of $0.1 \mathrm{~km} \mathrm{~s}^{-1}$ was added (see Sect. 3.2.1).

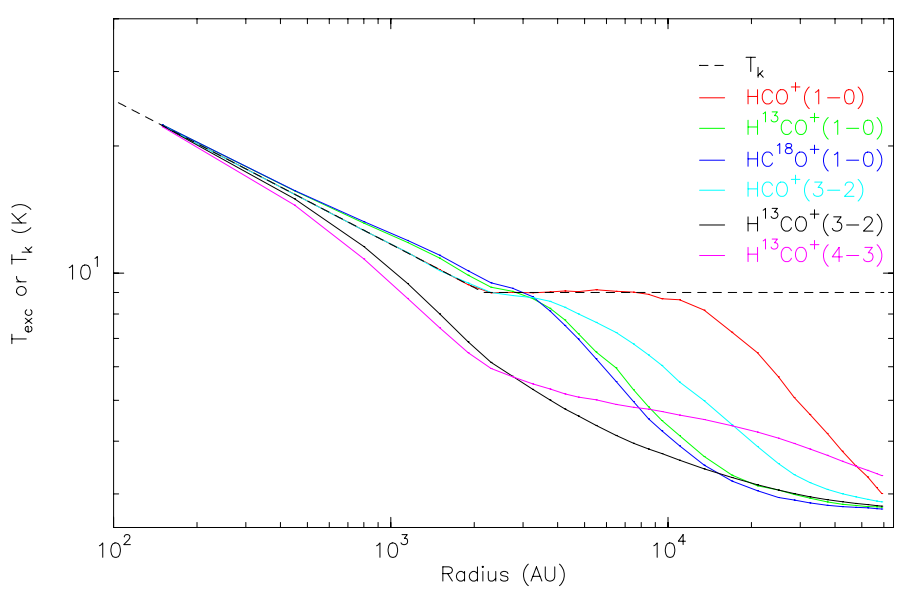

Fig. 16. Same as Fig. 13 for the best fit model "MHCOP". 


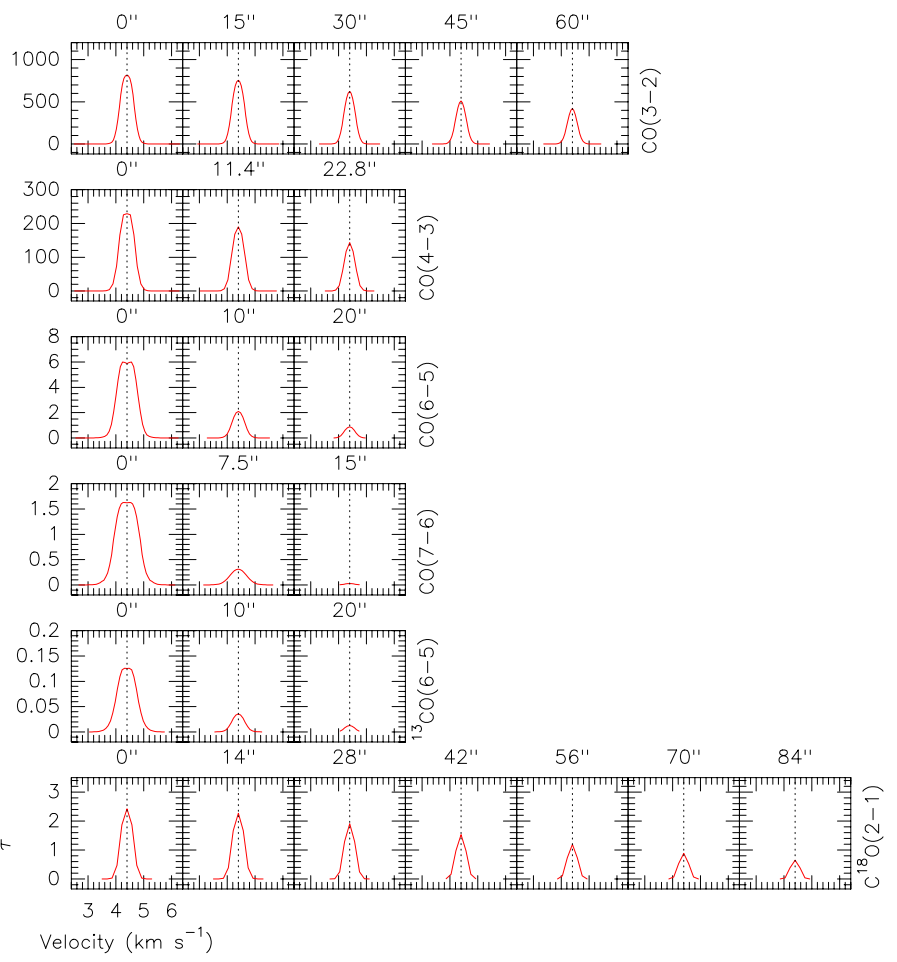

Fig. 18. Transition opacities for the best fit model "MCO". The dotted line shows the systemic velocity of Cha-MMS1. For the APEX spectra, a correction of $0.1 \mathrm{~km} \mathrm{~s}^{-1}$ was added (see Sect. 3.2.1).

\section{Appendix A: Mopra calibration and efficiency}

\section{A.1. Calibration ambiguities}

Some of our Mopra data show differences in the peak temperatures of the two polarisations, POL0 and POL1. The differences are not systematic for all the transitions observed. The two polarisations in some cases differ up to $\sim 10 \%$. The CS $2-1$ POL1 intensity for position P6 is stronger by $\sim 5 \%$ within the uncertainties, while $\mathrm{C}^{34} \mathrm{~S} 2-1$ has a stronger POL0 intensity by $\sim 9 \%$. POL0 also shows higher intensities for positions P1 and P6 of the $\mathrm{N}_{2} \mathrm{H}^{+} 1-0$ transition by $\sim 7 \%$ and $\sim 6 \%$, respectively. Most of the other transitions (and positions) do not show any significant differences. As the observed discrepancies are not systematic and are only seen in very few cases, we use the average of both polarisations per transition for the analysis in this paper.

Pronounced differences in intensity are seen when comparing a pair of spectra belonging to the same transition that was observed with different tuning frequencies. $\mathrm{HC}_{3} \mathrm{~N} 10-9$ and HNC 1-0 are the two transitions observed in both setups and show intensity differences of $\sim 38 \%$ and $\sim 33 \%$. Unfortunately, we have not yet found a satisfactory explanation for these discrepancies.

\section{A.2. Efficiency}

Even though the expected Mopra beam efficiency at the $90 \mathrm{GHz}$ band is $\sim 0.5^{9}$, we derive a value that is lower by $\sim 30 \%$ after performing independent calibration tests. IRAM 04191 in Taurus and OPH A SM1N in Ophiuchus were observed with both the

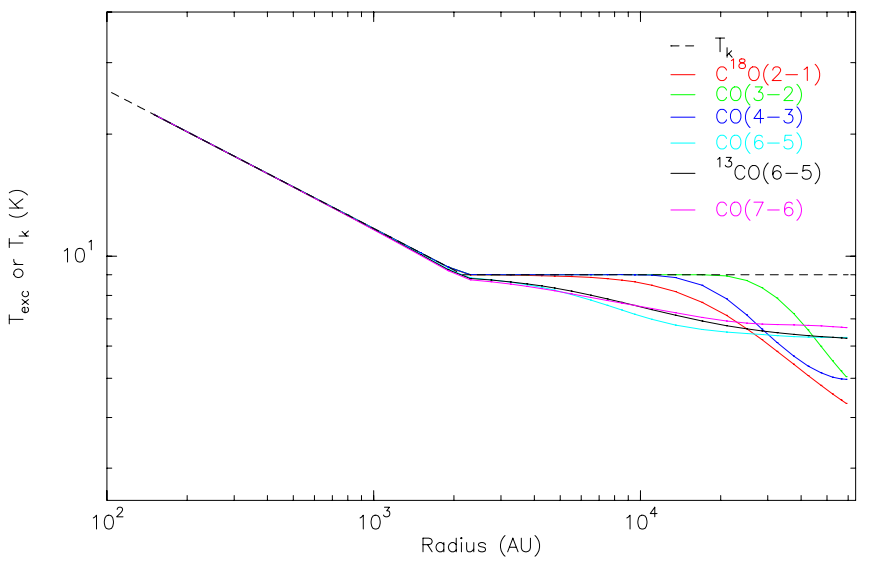

Fig. 19. Same as Fig. 13 for the best-fit model "MCO".

Table A.1. Mopra beam efficiencies.

\begin{tabular}{lcc}
\hline \hline Source & Line & Mopra efficiency \\
\hline IRAM 04191 & $\mathrm{N}_{2} \mathrm{H}^{+} 1-0$ & $0.25 \pm 0.01$ \\
IRAM 04191 & $\mathrm{CS} \mathrm{2-1}$ & $0.35 \pm 0.02$ \\
IRAM 04191 & $\mathrm{C}^{34} \mathrm{~S} 2-1$ & $0.52 \pm 0.13$ \\
IRAM 04191 & $\mathrm{H}^{13} \mathrm{CO}^{+} 1-0$ & $0.36 \pm 0.03$ \\
OPH A SM1N & $\mathrm{N}_{2} \mathrm{H}^{+} 1-0$ & $0.36 \pm 0.003$ \\
\hline
\end{tabular}

Notes. Mopra efficiencies derived after comparing independent observations from the IRAM $30 \mathrm{~m}$ and Mopra telescopes. The IRAM $30 \mathrm{~m}$ spectra were smoothed to the Mopra angular resolution.

Mopra and the IRAM $30 \mathrm{~m}$ telescopes (Belloche et al. 2002; André et al. 2007). IRAM 04191 was observed in the molecular transitions $\mathrm{CS} 2-1, \mathrm{~N}_{2} \mathrm{H}^{+} 1-0, \mathrm{C}^{34} \mathrm{~S} 2-1$, and $\mathrm{H}^{13} \mathrm{CO}^{+} 1-0$, while OPH A SM1N was observed in $\mathrm{N}_{2} \mathrm{H}^{+} 1-0$. After smoothing the $30 \mathrm{~m}$ data to the Mopra angular resolution we directly compared their integrated intensities with the equivalent Mopra intensities. We derived lower efficiencies for most of the observed transitions (see Table A.1) with the overall weighted average efficiency being $\sim 0.34$.

\section{Appendix B: Calculation of opacities}

\section{B.1. Opacity of the $\mathrm{C}^{18} \mathrm{O} 2-1$ line}

We observed both $\mathrm{C}^{18} \mathrm{O} 2-1$ and $\mathrm{C}^{17} \mathrm{O} 2-1$ at 13 positions perpendicular to the filament (Fig. 1). An isotopic ratio of $\left[{ }^{18} \mathrm{O}\right] /\left[{ }^{17} \mathrm{O}\right] \sim 4.11$ was found for the nearby $(140 \mathrm{pc})$ low-mass cloud $\rho$ Ophiuchus (Wouterloot et al. 2005). We use this value to derive the opacity of $\mathrm{C}^{18} \mathrm{O} 2-1$ using the following relation:

$\frac{I_{C^{18} O}}{I_{C^{17} O}}=\frac{1-\mathrm{e}^{-\tau} C^{18} O}{1-\mathrm{e}^{-\tau} C^{17} O}$

where $\mathrm{I}_{C^{18} O}$ and $\mathrm{I}_{C^{17} O}$ are the intensities of the two transitions, $\tau_{C^{18} O}$ and $\tau_{C^{17} O}$ their opacities and $\tau_{C^{17} O}=\tau_{C^{18} O} / 4.11$. We find opacities that gradually increase from $\leq 0.5$ at the outermost position to $\sim 1.6$ at the centre of the core for the $\mathrm{C}^{18} \mathrm{O} 2-1$ transition.

\footnotetext{
9 See http://www.narrabri.atnf.csiro.au/mopra/obsinfo. html
} 